Argonne

\title{
Demonstration of NEAMS Multiphysics Tools for Fast Reactor Applications
}

Nuclear Science and Engineering Division 
About Argonne National Laboratory

Argonne is a U.S. Department of Energy laboratory managed by UChicago Argonne, LLC under contract DE-AC02-06CH11357. The Laboratory's main facility is outside Chicago, at 9700 South Cass Avenue, Argonne, Illinois 60439. For information about Argonne and its pioneering science and technology programs, see www.anl.gov.

\title{
DOCUMENT AVAILABILITY
}

Online Access: U.S. Department of Energy (DOE) reports produced after 1991 and a growing number of pre-1991 documents are available free at OSTI.GOV (http://www.osti.gov/), a service of the US Dept. of Energy's Office of Scientific and Technical Information.

\author{
Reports not in digital format may be purchased by the public from the \\ National Technical Information Service (NTIS): \\ U.S. Department of Commerce \\ National Technical Information Service \\ 5301 Shawnee $\mathrm{Rd}$ \\ Alexandria, VA 22312 \\ www.ntis.gov \\ Phone: (800) 553-NTIS (6847) or (703) 605-6000 \\ Fax: (703) 605-6900 \\ Email: orders@ntis.gov
}

Reports not in digital format are available to DOE and DOE contractors from the Office of Scientific and Technical Information (OSTI):

U.S. Department of Energy

Office of Scientific and Technical Information

P.O. Box 62

Oak Ridge, TN 37831-0062

www.osti.gov

Phone: (865) 576-8401

Fax: (865) 576-5728

Email: reports@osti.gov

\section{Disclaimer}

This report was prepared as an account of work sponsored by an agency of the United States Government. Neither the United States Government nor any agency thereof, nor UChicago Argonne, LLC, nor any of their employees or officers, makes any warranty, express or implied, or assumes any legal liability or responsibility for the accuracy, completeness, or usefulness of any information, apparatus, product, or process disclosed, or represents that its use would not infringe privately owned rights. Reference herein to any specific commercial product, process, or service by trade name, trademark, manufacturer, or otherwise, does not necessarily constitute or imply its endorsement, recommendation, or favoring by the United States Government or any agency thereof. The views and opinions of document authors expressed herein do not necessarily state or reflect those of the United States Government or any agency thereof, Argonne National Laboratory, or UChicago Argonne, LLC. 


\section{Demonstration of NEAMS Multiphysics Tools for Fast Reactor Applications}

prepared by

Emily R. Shemon, Yiqi Yu, and Taek K. Kim

Nuclear Science and Engineering Division, Argonne National Laboratory

August 31, 2020 



\section{EXECUTIVE ABSTRACT}

The SHARP toolkit is a high-fidelity reactor simulation tool developed under the U.S. Department of Energy, Office of Nuclear Energy Advanced Modeling and Simulation (NEAMS) Campaign. SHARP toolkit is comprised of the neutronics module PROTEUS thermal hydraulics module Nek5000, and structural mechanics module Diablo. During FY17 and FY18, the PROTEUS and Nek5000 components of SHARP were applied to solve challenging sodium-cooled fast reactor (SFR) problems. In particular, selected hot channel factors (HCF) for a prototype metal-fueled SFR design (the AFR-100) were analyzed in high fidelity, and the "SHARP zooming capability" for SFRs was developed and demonstrated to reduce computational expense for full core problems in cases where detailed data is needed in selected fuel assemblies.

After the previous success applying SHARP to challenging SFR problems, the focus in FY19 and FY20 expanded to additional fast reactor applications including lead cooled fast reactors (LFR) and sodium cooled fast reactors (SFR). The specific technical tasks were (1) assessment of hot channel factors for LFR, for which no data currently exists, and (2) demonstration of zooming capability in assemblies of the Versatile Test Reactor (VTR).

First-of-a-kind hot channel factor (HCF) estimation for LFR with high fidelity codes (PROTEUS/Nek5000) was successfully demonstrated in this study which began in FY19 and continued in FY20. Selected HCF were computed and compared with SFR data (AFR-100, EBR-II). The findings confirm that different reactor types, design parameters and uncertainties lead to different HCFs. Careful estimation of HCF for a specific design is necessary to obtain appropriate HCFs. In addition to improvement in HCF accuracy, high fidelity tools generate data to help the designer better understand the mechanism of the impact from these uncertainties. For example, the impact of cladding thickness manufacturing tolerance resulted in non-intuitive effects in the corner pins of the LFR assembly. This procedure of computing HCF using high fidelity models shows promise and flexibility for being repeated for any arbitrary reactor of choice. Along with the application on SFR and LFR, the capability of the tools has also been matured to deal with different reactor types and designs.

Progress was made towards extending the previously demonstrated SHARP zooming capability to non-fueled SFR assemblies. In particular, in FY19 a gamma transport capability was implemented in both high fidelity PROTEUS solvers in order to accurately account for heat deposition caused by gamma particles, which accounts for $\sim 10 \%$ of total core power. Neutronics verification cases were carried out for a candidate Versatile Test Reactor (VTR) design using the new gamma transport capability in PROTEUS. Comparisons were made with continuous energy MCNP calculations and shown to agree well. The models for the full core design with heterogeneous control and fuel assemblies is in progress for PROTEUS-SN and completed with MCNP. The MCNP power distributions were transferred to Nek5000 to perform thermal hydraulic calculations of the control and fuel assembly. 


\section{TABLE OF CONTENTS}

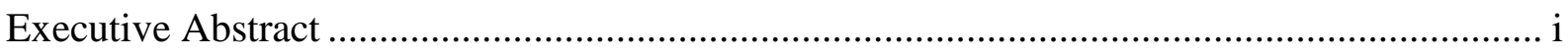

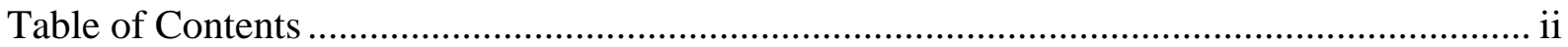

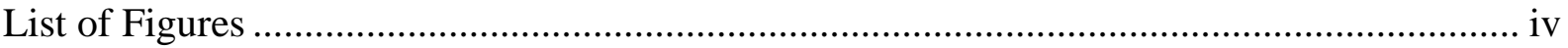

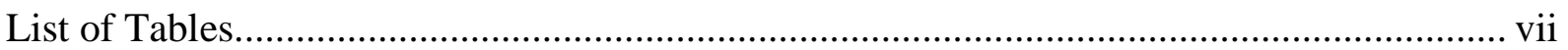

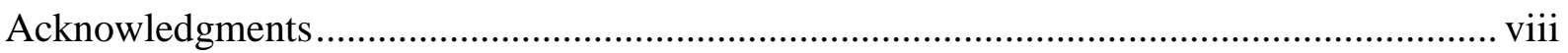

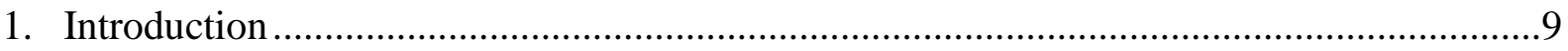

2. Assessment of Hot Channel Factors for a Lead-Cooled Fast Reactor Design.....................10

2.1 Westinghouse Lead-Cooled Fast Reactor Design ..........................................................10

2.2 Selection of LFR Hot Channel Factors …………...................................................13

2.3 Computational Tools and Resources.......................................................................

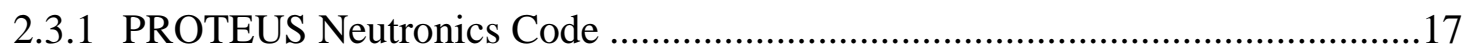

2.3.2 Nek5000 Thermal Hydraulics Code ................................................................17

2.3.3 Multiphysics Coupling Approach and Computational Resources ......................19

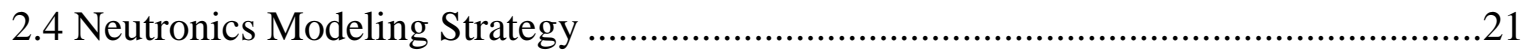

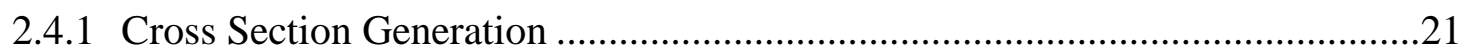

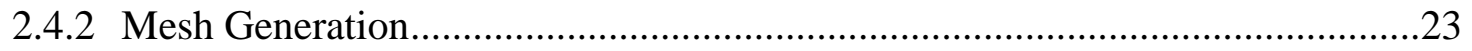

2.4.3 Neutronics Discussion of Nominal Condition..................................................23

2.5 Thermal Fluid Modeling Strategy ………………….............................................28

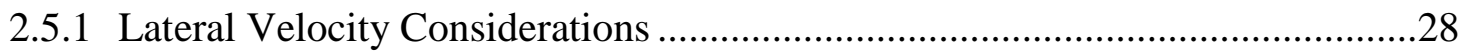

2.5.2 Turbulent Prandtl Number Consideration ...................................................30

2.6 HCF Associated with Uncertainties in Cladding Thickness .........................................36

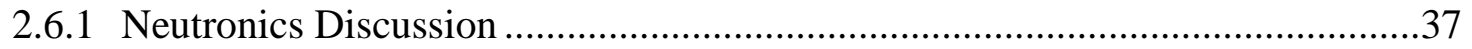

2.6.2 Thermal Hydraulics Discussion....................................................................

2.7 HCF Associated with Uncertainties in Fuel Pellet Fissile Content...............................41

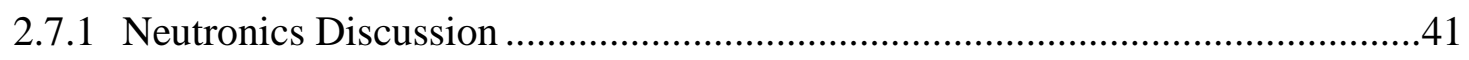

2.7.2 Thermal Hydraulics Discussion.................................................................46

2.8 HCF Associated with Uncertainties in Coolant Specific Heat......................................50

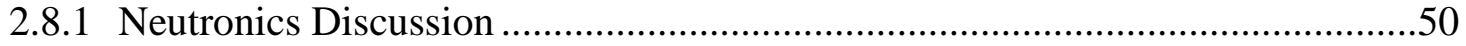

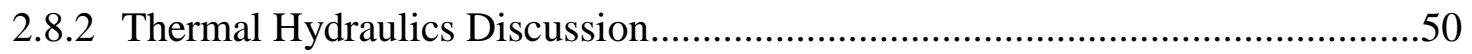

2.9 HCF Associated with Uncertainties in Coolant Density...............................................51 


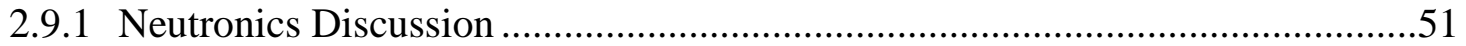

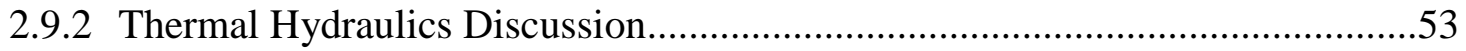

$2.10 \mathrm{HCF}$ Associated with Uncertainties in Coolant Isotopics ....................................53

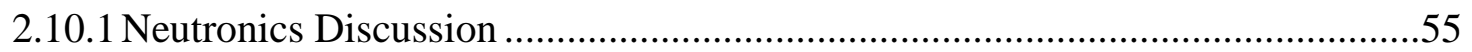

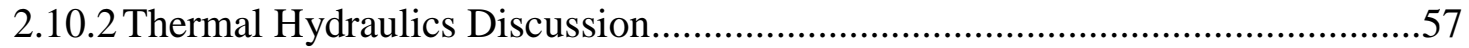

2.11 HCF Associated with Uncertainties in Cladding Thermal Conductivity..................57

2.12 HCF Associated with Uncertainties in Fuel Thermal Conductivity .......................58

$2.13 \mathrm{HCF}$ Associated with Uncertainties in Gap Conductance ....................................58

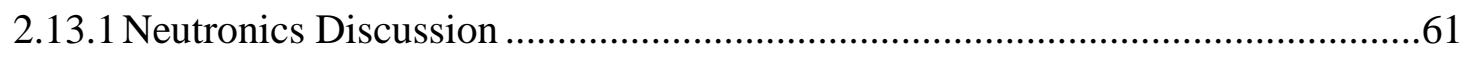

2.13.2 Thermal Hydraulics Discussion.....................................................................62

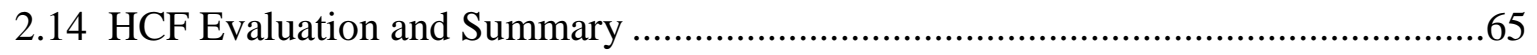

3. Zooming Capability for the Versatile Test Reactor ...................................................70

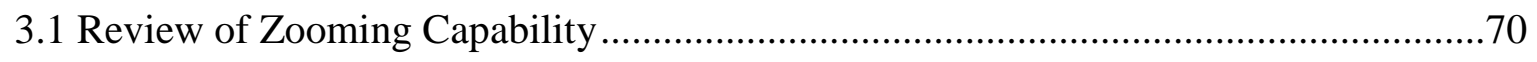

3.2 Description of VTR Zooming Objectives ........................................................71

3.3 VTR Model Description (see disclaimer at beginning of chapter) ............................71

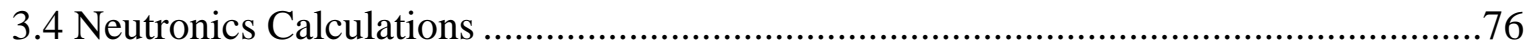

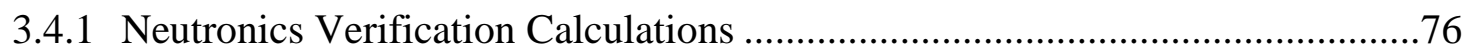

3.4.2 Progress on Heterogeneous Assembly Calculations ....................................82

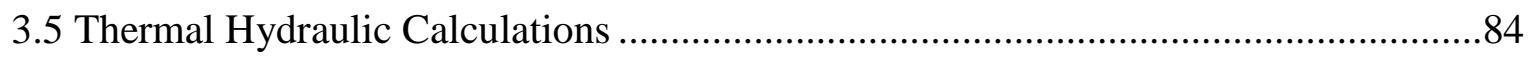

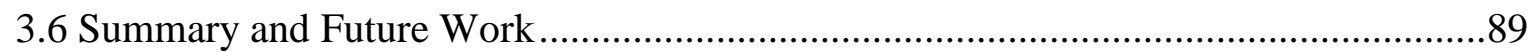

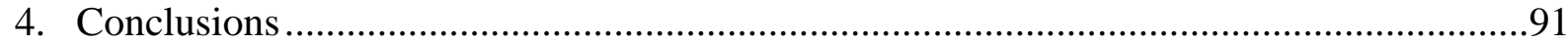

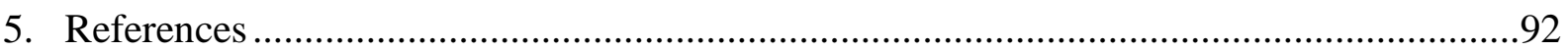




\section{LIST OF FIGURES}

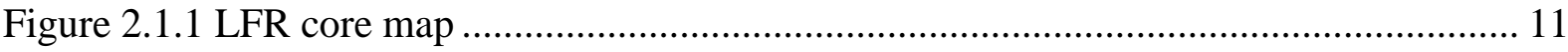

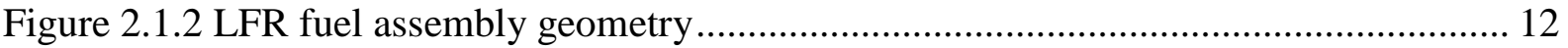

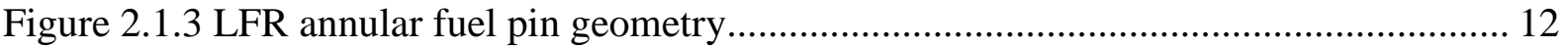

Figure 2.2.1 Schematic of assessment of margin to fuel-cladding eutectic formation ............ 14

Figure 2.2.2 SFRs operated or designed in the United States ............................................ 15

Figure 2.3.1 Comparison of convergence behavior between Nek5000 and OpenFOAM

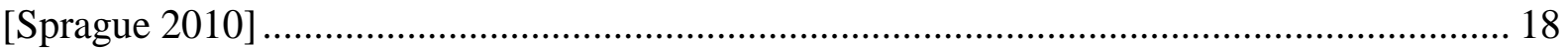

Figure 2.3.2 Hierarchical structure of NEAMS TH tools ................................................ 19

Figure 2.3.3 Schematic of offline coupling using components of the SHARP toolkit........... 20

Figure 2.4.1 Cross section generation procedure for heterogeneous 3D pin configurations ... 22

Figure 2.4.2 Conversion of assembly into 1-D cylinder geometry (not to scale) .................. 22

Figure 2.4.3 Top view of LFR neutronics mesh (left) and zoomed in (right) ...................... 24

Figure 2.4.4 Code-to-code verification of center pin power shape ..................................... 26

Figure 2.4.5 Left to right: Power distributions shown at bottom $(\mathrm{z}=135 \mathrm{~cm})$, middle $(\mathrm{z}=187$

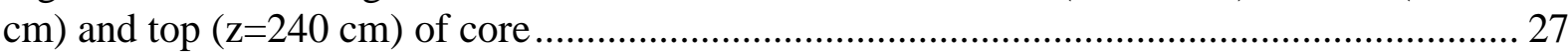

Figure 2.4.6 Axial power shapes in fuel zone of pin 1 (center) and pin 122 (outside corner). 27

Figure 2.5.1 Lateral velocity on different elevations of the LFR assembly......................... 28

Figure 2.5.2 Transient lateral velocity near the corner of the LFR assembly ....................... 29

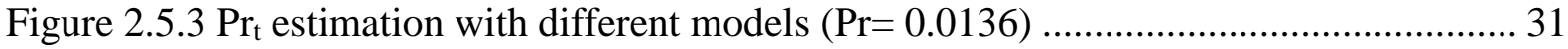

Figure 2.5.4 Computational domain, Boundary conditions and Mesh for LES ..................... 32

Figure 2.5.5 Nusselt number prediction with different models ....................................... 33

Figure 2.5.6 Temperature distribution between rods with varying turbulent Prandtl number. 35

Figure 2.5.7 Flow oscillation in the gap with different Reynolds number ........................... 36

Figure 2.6.1 Nominal and perturbed axial power shapes in pin 122 (outside corner) ............ 38

Figure 2.6.2 Nominal and perturbed integrated fuel powers (all pins) ............................... 39

Figure 2.6.3 Nominal and perturbed integrated cladding powers (all pins).......................... 39

Figure 2.6.4 Numbering scheme of fuel assembly .................................................... 40

Figure 2.6.5 Maximum coolant temperature responding to the uncertainty of cladding

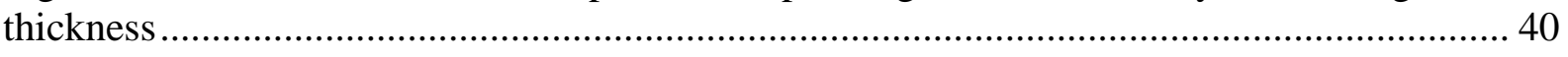

Figure 2.7.1 Distribution of eigenvalues across 30 random samples................................. 42

Figure 2.7.2 Power distribution at core mid-plane in nominal condition (left) and randomized fissile content bounding case in Simulation 2 (right). 43 
Figure 2.7.3 Distribution of enrichment perturbations for pins in Simulation 2 (simulation with maximum pin power)

Figure 2.7.4 Distribution of pin powers in Simulation 2 (simulation with maximum pin

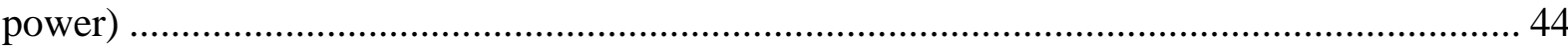

Figure 2.7.5. Normalized axial power distribution for selected pins within Simulation 2 ...... 45

Figure 2.7.6 Hypothetical peak pin power distribution based on random sampling with a very large number of samples. 46

Figure 2.7.7 Distribution of peak power and maximum cladding temperature ....................... 47

Figure 2.7.8 Maximum coolant temperature and peak power for all 30 cases ........................ 47

Figure 2.7.9 Maximum cladding temperature and peak power for all 30 cases ...................... 47

Figure 2.7.10 Maximum fuel temperature rise and peak power for all 30 cases ..................... 48

Figure 2.7.11 Location of maximum coolant temperature for Case 2 .................................... 49

Figure 2.7.12 Location of maximum cladding temperature for Case 2 ................................. 49

Figure 2.7.13 Location of maximum fuel temperature for Case 2 ………………............... 50

Figure 2.9.1 Relative difference in integrated pin power (reduced lead density case vs.

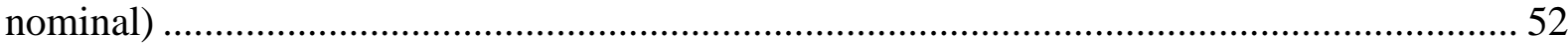

Figure 2.9.2 Relative difference in normalized axial power, center pin (reduced lead density case vs. nominal)

Figure 2.10.1 Relative difference in integrated pin power (high/low quality lead cases vs.

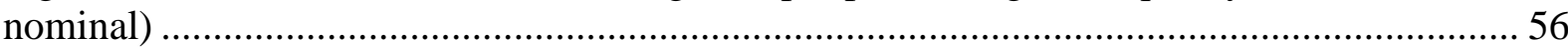

Figure 2.10.2 Relative difference in normalized axial power, center pin (high/low quality lead

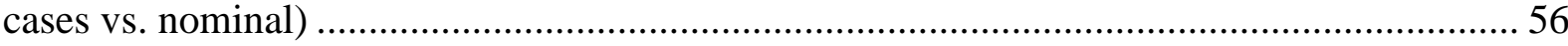

Figure 2.11.1 Maximum cladding temperature responding to the uncertainty of cladding

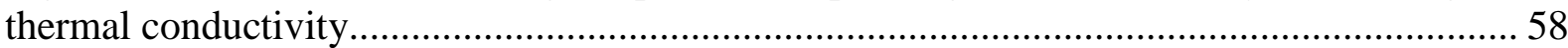

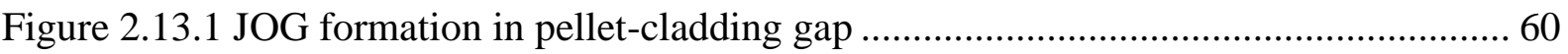

Figure 2.13.2 Gap conductance change along with the increase of burnup.............................. 60

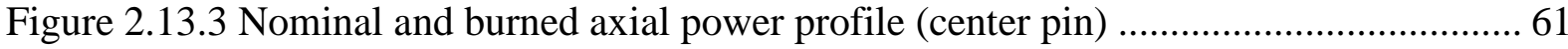

Figure 2.13.4 The effect of irradiation on the fuel-clad gap (top) and CFD model (bottom).. 63

Figure 2.13.5 Outline of thermal computation in the pellet-cladding gap with JOG................ 63

Figure 2.13.6 Gap conductance improvement due to the JOG formation ................................ 64

Figure 3.1.1 AFR-100 neutronics modeling for SHARP zoom calculation ............................ 71

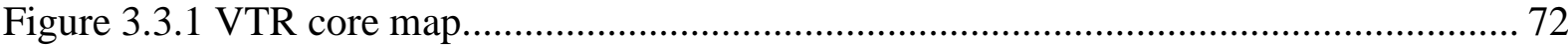

Figure 3.3.2 VTR core map showing focal assembly locations and meshes ........................... 73

Figure 3.3.3 VTR control assembly neutronics mesh (slice) ................................................... 74 
Figure 3.3.4 VTR fuel assembly neutronics mesh (slice)

Figure 3.4.1 Verification of axial power profile in fuel assembly (homogeneous full core, control inserted) 78

Figure 3.4.2 Verification of axial power profile in control assembly (homogeneous full core, control inserted) 78

Figure 3.4.3 PROTEUS-SN and MCNP radial power profiles (homogeneous full core, control inserted) 79

Figure 3.4.4 PROTEUS-SN and MCNP radial power profiles (homogeneous full core, control withdrawn) 80

Figure 3.4.5 Relative error in radial power profile in core (homogeneous full core, control inserted).

Figure 3.4.6 Relative error in radial power profile in core (homogeneous full core, control withdrawn) 81

Figure 3.4.7 Full core mesh with detailed fuel assembly. 82

Figure 3.4.8 Full core mesh with detailed fuel assembly (zoomed on fuel assembly) 83

Figure 3.4.9 Full core mesh with detailed control assemblies . 83

Figure 3.4.10 Full core mesh with detailed control assembly (zoomed on control assembly) 84

Figure 3.5.1 Thermal hydraulics mesh for control assembly (top view) 85

Figure 3.5.2 Temperature distribution of top-most control assembly based on MCNP power

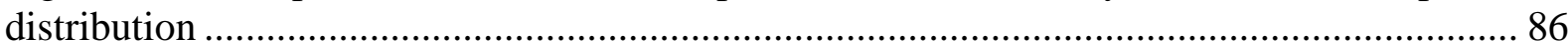

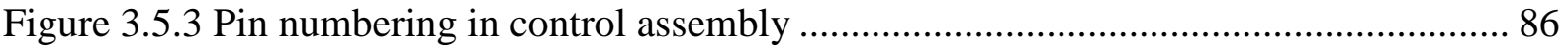

Figure 3.5.4 Maximum cladding temperatures in control assembly (MCNP power) ............. 87

Figure 3.5.5 Maximum absorber temperatures in control assembly (MCNP power) ............. 87

Figure 3.5.6 Velocity and temperature distribution in fuel assembly (MCNP power) ........... 88

Figure 3.5.7 Maximum cladding temperatures in fuel assembly (MCNP power) ................. 89

Figure 3.5.8 Maximum fuel temperatures rise in fuel assembly (MCNP power) ................... 89 


\section{LIST OF TABLES}

Table 2.1.1 Key parameters of Lead-Cooled Fast Reactor (LFR) model ................................ 10

Table 2.1.2 Axial LFR zones and neutronics modeling information...................................... 13

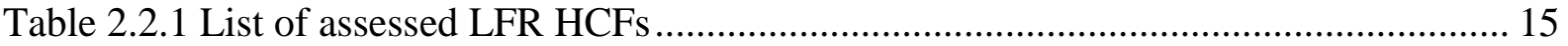

Table 2.3.1. Required high-fidelity calculations for HCF evaluations .................................... 21

Table 2.4.2. Code-to-code eigenvalue verification of LFR-based problems ........................... 25

Table 2.5.1. Correlations for turbulent Prandtl Number ....................................................... 30

Table 2.5.2. Correlations for Nusselt Number ........................................................................ 33

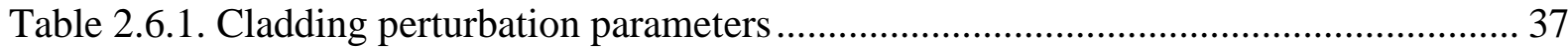

Table 2.7.1. Summary of fissile maldistribution impact on peak pin power .......................... 45

Table 2.7.2 Rank of peak power, coolant, cladding and fuel temperature rise for all 30 cases 48

Table 2.9.1 Comparison of neutronics for nominal and reduced lead density cases ............... 51

Table 2.10.1: Isotopic concentrations of various lead mines by percent [Watts 2013] ........... 54

Table 2.10.2. Impact of lead coolant properties on k-effective ................................................ 55

Table 2.13.1 Temperature rise with different gap conductance .................................................. 65

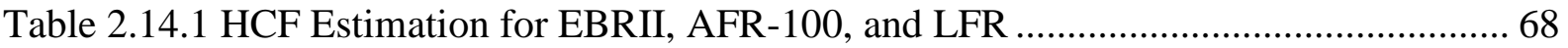

Table 2.14.2 Evaluation of LFR temperature rises (degrees K) in hot channels ...................... 68

Table 2.14.3 Equations to compute coolant, cladding, and fuel hot channel factors ............... 69

Table 3.3.1 VTR control assembly parameters ................................................................... 73

Table 3.3.2 VTR fuel assembly parameters .......................................................................... 74

Table 3.4.1 Eigenvalue verification for VTR cases .............................................................. 77 


\section{ACKNOWLEDGMENTS}

This research used resources of the Argonne Leadership Computing Facility, which is a DOE Office of Science User Facility supported under Contract DE-AC02-06CH11357. The authors thank the DOE ASCR Leadership Computing Challenge (ALCC) program for the 2019-2020 computing award on ALCF's Theta machine.

The authors gratefully acknowledge the computing resources provided on Bebop, a highperformance computing cluster operated by the Laboratory Computing Resource Center at Argonne National Laboratory.

The authors thank collaborators from Westinghouse Electric Company for valuable guidance and comments on the Lead-cooled Fast Reactor Hot Channel Factor calculations. The authors also thank Patrick Behne of Texas A\&M University for technical contributions to the neutronics verification simulations in this work. 


\section{Introduction}

The SHARP toolkit [Mahadevan 2014], [Merzari 2015] is a high-fidelity reactor simulation tool developed under the U.S. Department of Energy, Office of Nuclear Energy Advanced Modeling and Simulation (NEAMS) Campaign. SHARP toolkit is comprised of the neutronics module PROTEUS [Shemon 2014], thermal hydraulics module Nek5000 [Fischer 2008], and structural mechanics module Diablo.

During FY17 and FY18, SHARP was applied to solve challenging sodium-cooled fast reactor (SFR) problems [Shemon 2018]. In particular, selected hot channel factors (HCF) for a prototype metal-fueled SFR design (the AFR-100) [Grandy 2013][Kim 2012] were analyzed using the high fidelity codes PROTEUS and Nek5000. The SHARP-calculated hot channel factors were compared with legacy hot channel factors for the metal-fueled EBR-II reactor [Ku 1994] which were generated by a combination of low fidelity codes, empirical correlations, and mockup experiments. The findings of this study demonstrated that high fidelity modeling and simulation can offer a benefit in assessing hot channel factors for SFRs by (1) reducing modeling uncertainty due to the increased geometric and physics detail taken into account, and (2) eliminating the need for mockup experiments. Additionally, the "SHARP zooming capability" for SFRs was developed and demonstrated to reduce computational expense for full core problems in cases where detailed data is needed in only selected fuel assemblies. The zooming capability is enabled by a multi-resolution mesh in neutronics where high fidelity meshes are used only in the fuel assembly of interest. The power distribution is passed to the thermal fluids solver (Nek5000) which models only the assembly of interest due to sufficient isolation from neighboring assemblies by the duct walls.

After the success applying SHARP to challenging SFR problems in FY17 and FY18, the focus in FY19 and FY20 expanded to demonstrating SHARP's capability to target additional reactor types. In particular, the tasks performed in FY19 were: (1) Assessment of hot channel factors for a lead-cooled fast reactor (LFR) design, and (2) Demonstration of zooming capability in a fuel and control assembly of the Versatile Test Reactor (VTR). This report is a collective summary of work performed on these topics over FY19-FY20. 


\section{Assessment of Hot Channel Factors for a Lead-Cooled Fast Reactor Design}

This chapter discusses the assessment of hot channel factors (HCF) for a Westinghouse Electric Company (WEC) lead-cooled fast reactor design using the NEAMS-developed SHARP tools PROTEUS and Nek5000. The ducted assemblies of this particular design make the simulation requirements of the LFR similar to conventional SFR designs. Additionally, there are no known published HCF datasets for LFRs, making the Westinghouse LFR an ideal candidate for this analysis. Application of SFR-based HCF dataset to an LFR design is questionable, and so HCF analysis of LFR is needed. This task demonstrates the ability of the SHARP tools to perform such an analysis by assessing a limited list of LFR HCFs which were prioritized by WEC.

\subsection{Westinghouse Lead-Cooled Fast Reactor Design}

Westinghouse Electric Company (WEC), together with an international team, is developing its next generation high capacity nuclear power plant (NPP) based on lead-cooled fast reactor (LFR) technology. WEC provided technical details of their LFR design (current as of late 2018) and helped prioritize the hot channel factors to assess during FY19. All design details contained within this report are openly available [Grasso 2019].

Key parameters of the WEC LFR design are listed in Table 2.1.1. This medium-size, simple, scalable and passively safe plant harnesses a liquid lead-cooled, fast neutron spectrum core operating at high temperatures in a pool configuration reactor. The power output is $950 \mathrm{MWth}$ $(\sim 450 \mathrm{MWe})$. The core map is provided in Figure 2.1.1. The core consists of three concentric fuel assembly zones, two banks of control assemblies, safety, reflector and shield assemblies. In this work, hot channel factors are analyzed for the inner zone fuel assembly type only, a cross section of which is shown in Figure 2.1.2. As performed in conventional HCF calculations, the impact of neighboring assemblies is not accounted for in this analysis. The generated HCF are generic enough to be applied to specific pins or assemblies in a full core analysis to yield actual temperatures.

Table 2.1.1 Key parameters of Lead-Cooled Fast Reactor (LFR) model

\begin{tabular}{ccc}
\hline Parameter & Value & unit \\
\hline Thermal power & 950 & $\mathrm{MW}$ \\
Active core height & 105 & $\mathrm{~cm}$ \\
Number of fuel pins per assembly & 127 & - \\
Gap thickness & 0.175 & $\mathrm{~mm}$ \\
Duct thickness & 3.5 & $\mathrm{~mm}$ \\
Fuel pin diameter & 10.7 & $\mathrm{~mm}$ \\
Fuel pin pitch & 13.3 & $\mathrm{~mm}$ \\
P/D & 1.24 & -
\end{tabular}


Cladding thickness

Fuel pellet outer diameter

Fuel pellet inner diameter

Reynolds number

Prandtl number

Peclet number

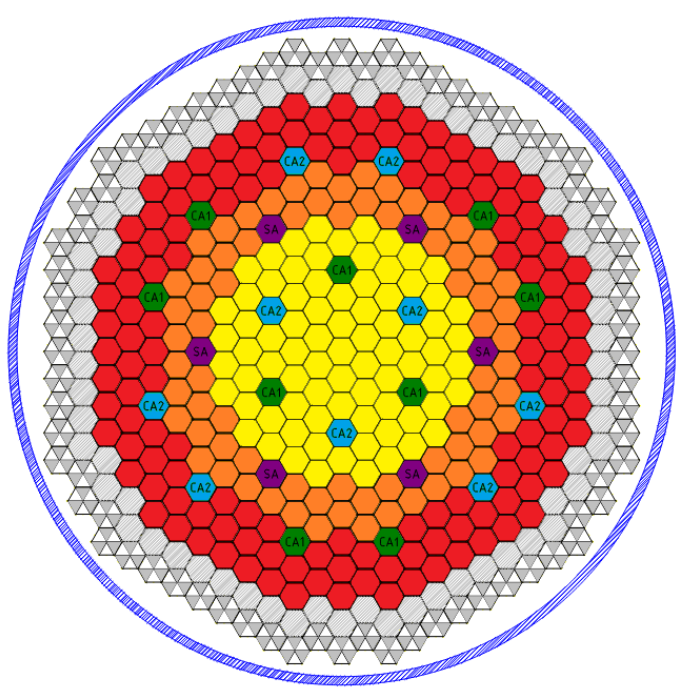

0.9

8.55

4.0

$5.1 \times 10^{4}$

0.0136

705.21 $\mathrm{mm}$

$\mathrm{mm}$

$\mathrm{mm}$

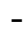

-

$-$

Figure 2.1.1 LFR core map

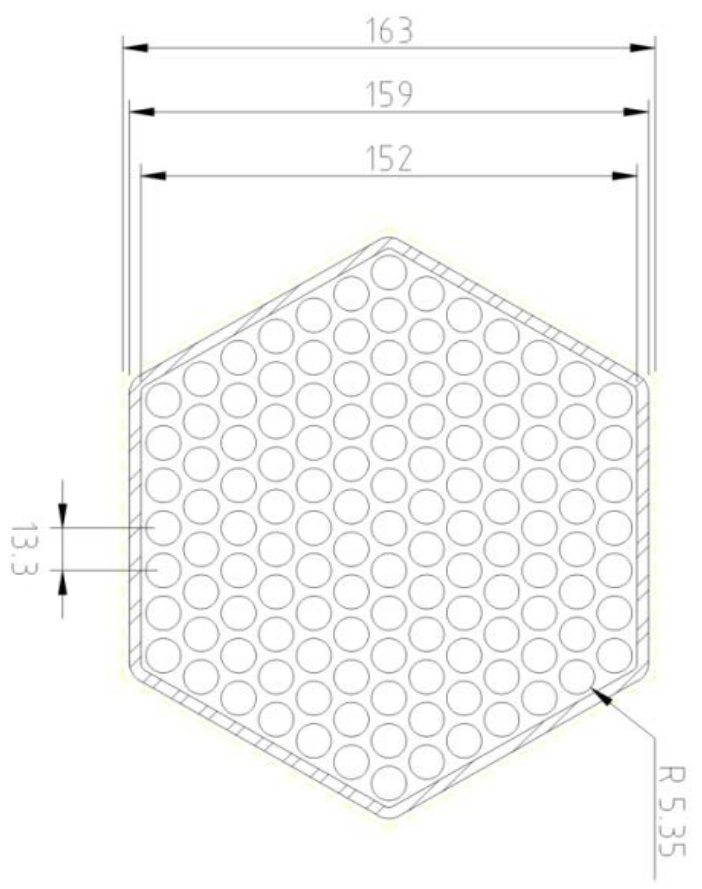




\section{Figure 2.1.2 LFR fuel assembly geometry}

The fuel assembly pitch is $16.3 \mathrm{~cm}$ with a $4 \mathrm{~mm}$ lead-filled gap between assemblies and 3.5 $\mathrm{cm}$ thick duct wall. Each assembly contains 127 cladded fuel pins arranged in a triangular lattice with pitch $1.33 \mathrm{~cm}$ within a hexagonal wrapper (duct). Each fuel pin has a cold fuel inner/outer diameter of 4.00/8.55 mm respectively, a fuel-cladding gap of $0.175 \mathrm{~mm}$ and a cladding outer diameter of $10.7 \mathrm{~mm}$ with a cladding thickness of $0.90 \mathrm{~mm}$, depicted in Figure 2.1.3. Helium gas is present in the empty regions. In order to minimize the flow speed and consequently mitigate corrosion issues, a relative wide lattice $(\mathrm{P} / \mathrm{D}=1.24)$ design is adopted. Grid spacers are planned to maintain pin spacing, rather than the wire wrap used in conventional SFR designs. Annular MOX fuel (UPuO) is envisaged for countries with spent fuel management policies supporting reprocessing and reuse of plutonium. Annular fuel is very beneficial for fast reactors because of its availability for both high power and high burnup. Most of the annular pellets irradiated up to high burnup showed central-hole shrinkage due to deformation and restructuring during irradiation. This shrinkage has a great influence on power-to-melt, which is a main factor in deciding the maximum power in the fuel design [Ikusawa 2017].

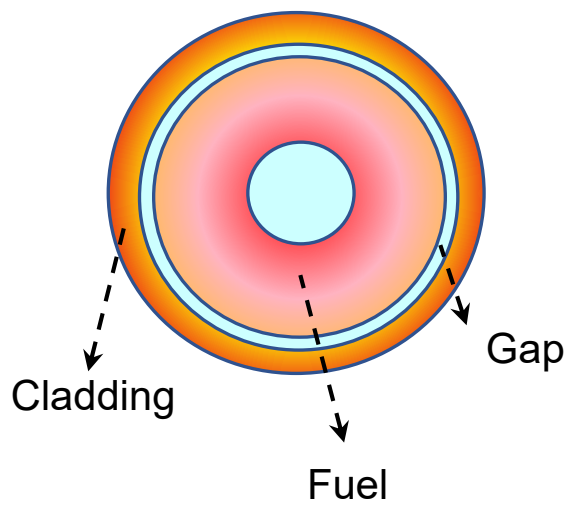

Figure 2.1.3 LFR annular fuel pin geometry

The MOX $(\mathrm{UPuO})$ fuel composition in the inner fuel zone is specified as a mixture of depleted uranium (0.25 wt \% U-235) and enriched plutonium (68.769 wt\% Pu-239, $1.759 \mathrm{wt} \% \mathrm{Pu}-241)$, with $23 \% \mathrm{PuO}_{\mathrm{x}} /\left(\mathrm{PuO}_{\mathrm{x}}+\mathrm{UO}_{\mathrm{x}}\right)$. The cold fuel density is $10.423 \mathrm{~g} / \mathrm{cc}$. The wrapper (duct) and pin cladding are made of DS4 with density $7.970 \mathrm{~g} / \mathrm{cc}$. The coolant is lead with density 10.402 $\mathrm{g} / \mathrm{cc}$. The axial regions of the fuel assembly are described in Table 2.1.2. The lower gas plenum through upper gas plenum are represented explicitly in neutronics (except for homogenizing the spring and tube), and regions above and below these are modeled homogeneously. Thermal expansion factors are applied to generate hot condition dimensions (1.021\% fuel, $0.937 \%$ DS4 wrapper, $1.005 \%$ DS4 cladding, and $0.715 \%$ AISI316). 
Table 2.1.2 Axial LFR zones and neutronics modeling information

\begin{tabular}{|c|c|c|c|}
\hline Axial Zone & Neutronics Model Description & $\begin{array}{l}\text { Upper Z } \\
\text { (cm) }\end{array}$ & $\begin{array}{l}\text { Neutronics XS } \\
\text { Temp. (K) }\end{array}$ \\
\hline Lower core plate & $\begin{array}{l}\text { Homogenized mixture AISI316, } \\
\text { DS4, } \mathrm{Pb}\end{array}$ & 10.0 & 693 \\
\hline Inlet wrapper & Homogenized mixture DS4, Pb & 40.5 & 693 \\
\hline Lower bundle grid/pins plug & Homogenized mixture DS4, $\mathrm{Pb}$ & 47.0 & 693 \\
\hline Lower gas plenum tube & $\begin{array}{l}\text { Heterogeneous DS4 wrapper \& } \\
\text { cladding, } \mathrm{Pb} \\
\text { Homogenized DS4/He tube }\end{array}$ & 132.0 & 693 \\
\hline Lower thermal insulator & Heterogeneous DS4, Pb, He, YSZ & 133.5 & 693 \\
\hline Active core & $\begin{array}{l}\text { Heterogeneous DS4, } \mathrm{Pb}, \mathrm{UPuO}, \mathrm{He} \text {, } \\
\mathrm{Pb}\end{array}$ & 238.5 & $\begin{array}{c}808 \text { (Wrapper, } \mathrm{Pb}) \\
843 \text { (Cladding) } \\
1300 \text { (He, UPuO) }\end{array}$ \\
\hline Upper thermal insulator & Heterogeneous DS4, Pb, He, YSZ & 240.0 & 923 \\
\hline Upper gas plenum spring & $\begin{array}{l}\text { Heterogeneous DS4 wrapper \& } \\
\text { cladding, Pb } \\
\text { Homogenized DS4/He spring }\end{array}$ & 252.0 & 923 \\
\hline Upper bundle grid/pins plug & Homogenized mixture DS4, $\mathrm{Pb}$ & 257.0 & 923 \\
\hline Outlet wrapper & Homogenized mixture DS4, Pb & 350.0 & 923 \\
\hline
\end{tabular}

\subsection{Selection of LFR Hot Channel Factors}

A detailed background of hot channel factors in the nuclear design community can be found in the SFR applications report from FY18 [Shemon 2018]. A general overview is given here for brevity.

Various uncertainties are involved in the predictions of reactor design parameters, such as theoretical and experimental analysis uncertainties, instrumentation uncertainties, manufacturing tolerances, correlation uncertainties, and method and simulation uncertainties. These uncertainties impact the peak cladding, fuel and coolant temperatures in the system above those expected in the nominal condition (no uncertainties). The peak temperatures in the as-built system must be nevertheless be maintained at safe margins away from maximum temperatures 
that could compromise the integrity and performance of the materials. Therefore, the impact of uncertainties on the temperature predictions is typically accounted for through the assessment of "hot channel factors", which take into account the increase in temperature due to specific uncertainties. Figure 2.2.1 illustrates the nominal cladding temperature (lowest value), the impact of modeling and simulation uncertainties (raises to the peak value), and the design limit (a safe margin away from the physical eutectic formation temperature). The goal of this work is to reduce or/and eliminate HCFs using high-fidelity multi-physics toolkit by better accounting for the uncertainties associated with HCFs in the early fast reactor designs in order that the nominal peak cladding temperature can be raised, resulting in higher power and greater economic gains.

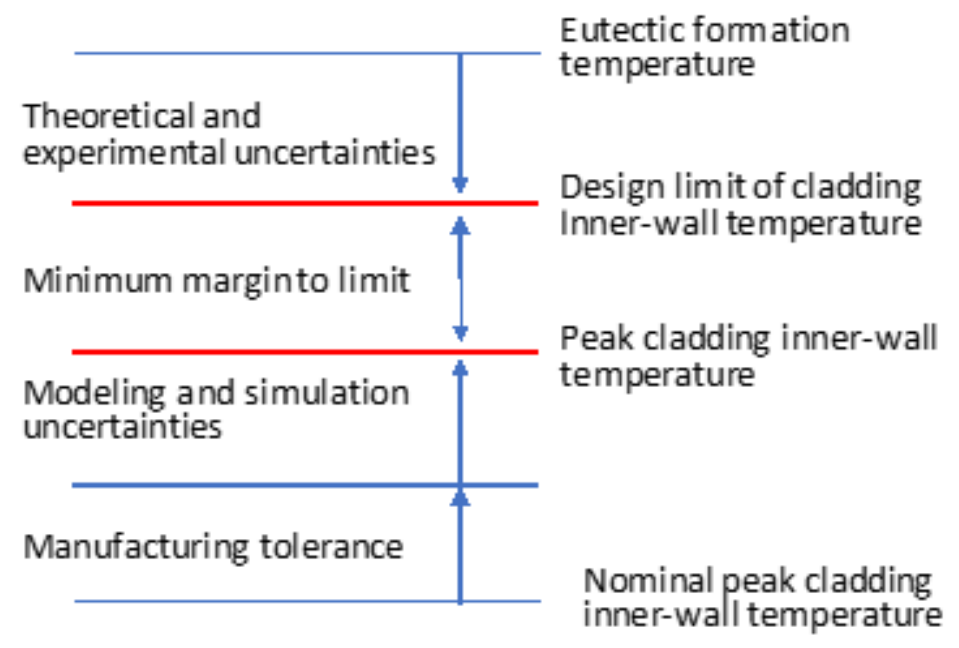

Figure 2.2.1 Schematic of assessment of margin to fuel-cladding eutectic formation

In the United States, four sets of HCFs have been developed under various SFR development programs, and the magnitudes of HCFs were strongly related to the status of modeling and simulation and computing power when the HCFs were developed. Figure 2.2.2 shows the SFR development, deployment, and operation history, including the activities on HCF development aimed at specific SFRs. A set of HCFs was developed for FFTF initially in 1976 and later updated in 1990. A significant effort to develop HCFs was conducted to support the licensing of Clinch River Breeder Reactor (CRBR) in 1980. The last set of HCFs was developed in 1995 under the Integral Fast Reactor (IFR) project to develop Mark-V metallic fuels in the EBR-II. However, the EBR-II and FFRF were permanently shut down, and SFR programs were canceled in the mid-1990s, and therefore no additional efforts were regarding HCFs since then, except for reviews of existing HCF sets for new SFRs. Therefore, the absence of any HCFs for LFR-based designs and questionable applicability of legacy SFR HCFs makes the LFR a good candidate for modern assessment of HCFs with the SHARP toolkit. 


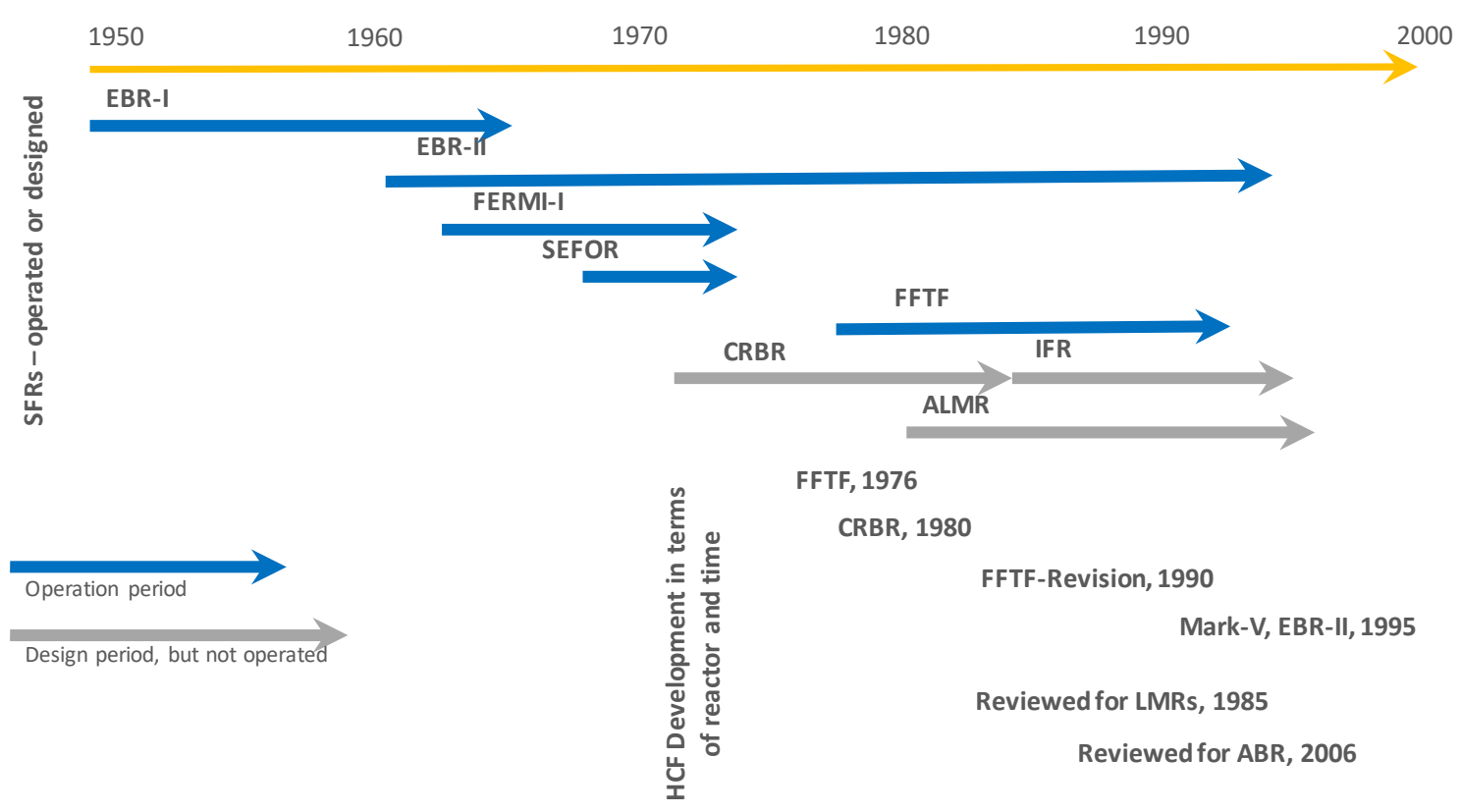

Figure 2.2.2 SFRs operated or designed in the United States

The following hot channel factors in Table 2.2.1 were considered during FY19, as prioritized by WEC given available computing time and code capabilities. WEC provided the associated tolerances and uncertainties. The modeling strategies and results for assessing each of the above uncertainties is discussed in individual sections.

\section{Table 2.2.1 List of assessed LFR HCFs}

\begin{tabular}{|l|l|}
\hline LFR Hot Channel Factor & Description \\
\hline $\begin{array}{l}\text { Cladding thickness } \\
\text { (subchannel flow area) }\end{array}$ & $\begin{array}{l}\text { Assess impact of variances in cladding thickness due to } \\
\text { manufacturing tolerance } \\
\text { Assumption: +/- } 0.05 \mathrm{~mm} \text { tolerance; change uniformly by } \\
\text { maximum value in all pins due to meshing complexity }\end{array}$ \\
\hline Fissile fuel maldistribution & $\begin{array}{l}\text { Assess impact of uncertainties in fissile content due to } \\
\text { manufacturing tolerance } \\
\text { Assumption: }+/-5 \% \text { tolerance on Pu-239 enrichment; sample } \\
\text { stochastically in all pins }\end{array}$ \\
\hline
\end{tabular}




\begin{tabular}{|c|c|}
\hline Coolant specific heat & $\begin{array}{l}\text { Assess impact of uncertainties in lead coolant specific heat } \\
\text { Assumption: }+/-5 \% \text { uncertainty in lead specific heat }\end{array}$ \\
\hline $\begin{array}{l}\text { Fuel thermal conductance } \\
\text { across pellet-cladding gap }\end{array}$ & $\begin{array}{l}\text { In low conductivity fuels with no bond, uncertainty exists for } \\
\text { the thermal conductance across the pellet cladding gap. } \\
\text { Estimate the sensitivity of the fuel temperature to the gap by } \\
\text { performing analysis at two bounding cases: assuming perfect } \\
\text { eccentricity of the fuel at (1) fresh fuel with open gap } \\
\text { (nominal condition), and (2) burned fuel with closed gap. } \\
\text { Assumption: Detailed data on uncertainties in joint-oxide gain } \\
\text { formation was not available, so the two bounding cases were } \\
\text { analyzed. To assess the HCF, detailed fuel models and } \\
\text { uncertainties are required which is outside the scope of the } \\
\text { current SHARP toolkit and work scope. }\end{array}$ \\
\hline Coolant density & $\begin{array}{l}\text { Assess impact of uncertainties in lead coolant density } \\
\text { Assumption: +/- } 0.8 \% \text { uncertainty in lead coolant density }\end{array}$ \\
\hline Coolant isotopics & $\begin{array}{l}\text { Assess impact of uncertainties in lead coolant isotopics. } \\
\text { Assumption: Use "low" quality and "high" quality lead with } \\
\text { varying Pb-208 contents to simulate lead sourced from } \\
\text { different mines. }\end{array}$ \\
\hline Cladding conductivity & $\begin{array}{l}\text { Assess impact of uncertainties in cladding conductivity. } \\
\text { Assumption: +/- } 10 \% \text { uncertainty in cladding conductivity }\end{array}$ \\
\hline Fuel conductivity & $\begin{array}{l}\text { Assess impact of uncertainties in fuel conductivity. } \\
\text { Assumption:+/- } 21.3 \% \text { uncertainty in fuel conductivity }\end{array}$ \\
\hline
\end{tabular}

\subsection{Computational Tools and Resources}

The SHARP Toolkit is a DOE NEAMS software product that performs highly detailed multiphysics calculations with online data transfer. In this section, two sub-components of the SHARP toolkit, PROTEUS and Nek5000, used to assess the LFR HCFs are briefly described, including the overall computation approach by coupling two component codes and computation resources. 


\subsubsection{PROTEUS Neutronics Code}

PROTEUS has two high fidelity neutronics solvers, PROTEUS-SN and PROTEUS-MOCEX. The PROTEUS-MOCEX solver was used for the HCF calculations. The PROTEUS-SN code was used for the VTR zooming calculations so information is included here as well.

The PROTEUS-MOCEX code solves the method of characteristics equations in the 2D plane for an unstructured mesh and solves the exact equations in the axial plane using the discontinuous Galerkin finite element method. The method is also known as "extruded MOC" as it relies on an extruded 2D mesh in the axial plane for geometry description. The method is more accurate than other extruded MOC methods such as DeCart and MPACT which rely on the 2D/1D synthesis method and can suffer from convergence and accuracy issues in certain core geometries. It is generally more memory intensive than the PROTEUS-SN code but uses less cores and less compute time, typically, to solve the same problem.

The PROTEUS-SN solver was a previous Gordon Bell Finalist for computational performance on the BlueGene/P (BG/P) architecture. It solves the second order, even parity form of the neutron transport equation using discrete ordinates approximation in angle, unstructured continuous finite element method in space, and multigroup approximation in energy. The code relies on the PETSc linear solver package for performing large scale matrix solutions of the underlying eigenvalue problem, the METIS package for online mesh partitioning, and the HDF5 package for detailed output. Parallelism is performed with MPI, and depending on the memory requirements for the cross section data, typically $1 \mathrm{MPI}$ rank/core is optimal and reasonable. The code does not use multithreading at this point as threading is not particularly useful for the matrix operations.

Both PROTEUS-SN and PROTEUS-MOCEX have been studied for benchmark problems and compared well to Monte Carlo (MCNP or SERPENT) models as well as some limited validation data (ZPR, TREAT). However, the accuracy of high fidelity deterministic codes such as these is highly dependent on the accuracy of the multigroup constants fed into the codes as inputs. Significant research has been performed to assess the procedures for generating multigroup cross section data (an input parameter to deterministic neutronics codes like PROTEUS-SN and PROTEUS-MOCEX) for explicit pin-by-pin geometries [Hader 2014], [Jarrett 2016]. The techniques learned in the past few years have been implemented in this work.

\subsubsection{Nek5000 Thermal Hydraulics Code}

Nek5000 is an open source code designed to simulate unsteady incompressible Navier-Stokes flow, low Mach-number flows flow, heat transfer and species transport and incompressible magneto hydrodynamics (MHD). Nek5000 is a previous winner of the Gordon Bell Prize in high performance computing and continues to adapt to new architectures with its highly scalable algorithms. Its fast scalable multigrid solver can scale to more than 290,000 processors on the 
$\mathrm{BG} / \mathrm{P}$ architecture using pure MPI with multiple ranks per core, and beyond 1M MPI ranks on $\mathrm{BG} / \mathrm{Q}$ architecture at $60 \%$ efficiency with only $2000 \mathrm{DOFs} / \mathrm{rank}$.

Nek5000 is based on a high-order spectral element method and achieves extremely rapid (exponential) convergence at low cost. Figure 2.3.1 shows the comparison of convergence behavior between Nek5000 and OpenFOAM (another well-known open source CFD code), where Nek5000 has a remarkable advantage on convergence behavior (fewer DOF are required for a desired accuracy). In addition to its high-order foundation, Nek5000 has several other features that make it ideally suited for large-scale parallel simulations. Temporal discretization is based on a high-order splitting that is third order accurate in time and reduces the coupled velocity-pressure Stokes problem to independent three Helmholtz and one elliptic solver per time step, i.e. for each velocity component and pressure, respectively. The velocity problems are diagonally dominant and thus easily solved by using Jacobi preconditioned conjugate gradient iteration. The pressure sub-step requires a Poisson solve at each step, which is performed through multigrid-preconditioned GMRES iteration coupled with temporal projection to find an optimal initial guess. Particularly important components of Nek5000 are its scalable coarse-grid solvers that are central to parallel multigrid. Nek5000 has been extensively tested for over 25 years.

More than 150 journal articles have been published by worldwide users of Nek5000. More than 400 tests have been performed to validate the models in the code in different research fields. The user community includes over 400 scientists and engineers in academia, laboratories and industry. The Nek5000 has been used for numerous INCITE and ALCC awards at the ALCF in recent years. All required physics modules for this work are already included in Nek5000. Figure 2.3.2 shows a schematic representation of the full hierarchy of thermal hydraulic codes in NEAMS toolkit. Given the computational cost, we focus on RANS-based CFD simulations for this work.

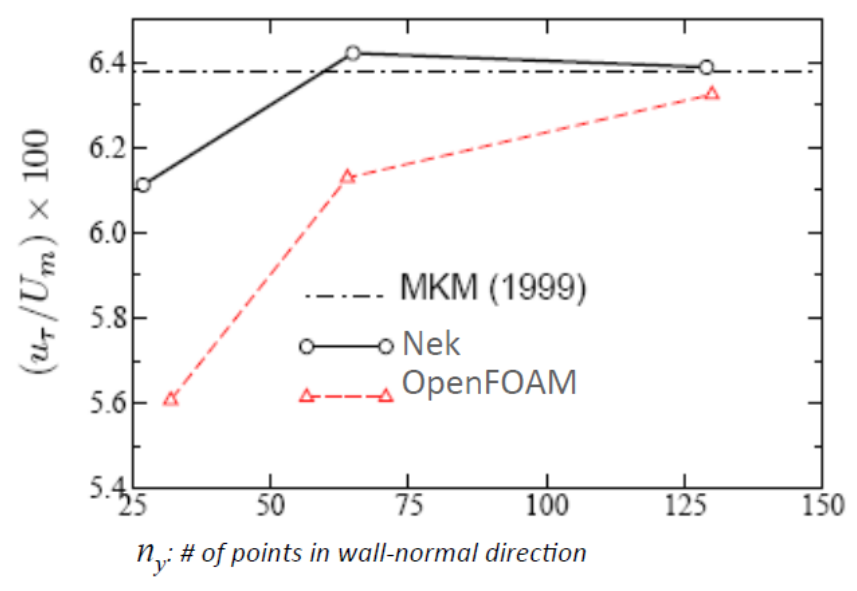

Figure 2.3.1 Comparison of convergence behavior between Nek5000 and OpenFOAM [Sprague 2010] 


\section{Deviation}

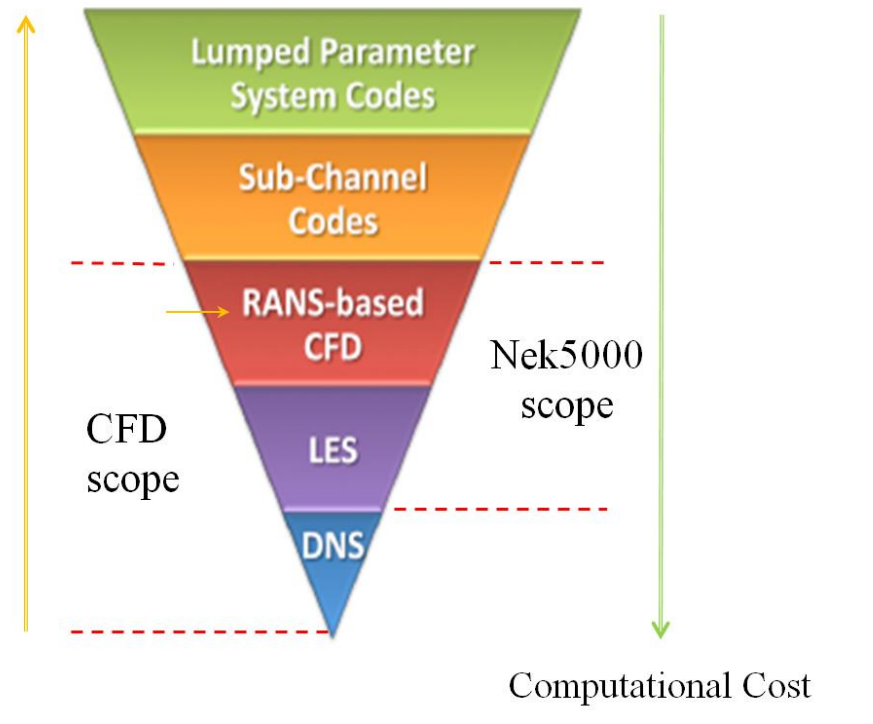

Figure 2.3.2 Hierarchical structure of NEAMS TH tools

\subsubsection{Multiphysics Coupling Approach and Computational Resources}

PROTEUS calculates the detailed power density and flux distributions in a nuclear reactor core. Nek5000 calculates the detailed velocity and temperature distributions in a nuclear reactor core. In FY16, a tightly coupled version of SHARP was released in which PROTEUS and Nek5000 transmit data (temperature, power) in memory via two-way iteration between the two physics on a fine grained (element-wise) basis. However, some technical limitation make it not suitable for performing large simulations.

The offline coupling scheme developed in FY17-FY18 for the SFR HCF assessment, shown in Figure 2.3.3, was again utilized for this work. The offline coupling scheme allows the PROTEUS-MOCEX solver (more efficient for the proposed single assembly calculations) to be used along with Nek5000 and also allows each code to choose its own optimal parallelization scheme, providing much greater computational flexibility and efficiency. The drawback of offline coupling is the coarser-grained data transfer as well as the manual interventions that are required between code iterations that preclude efficient 2-way coupling. 


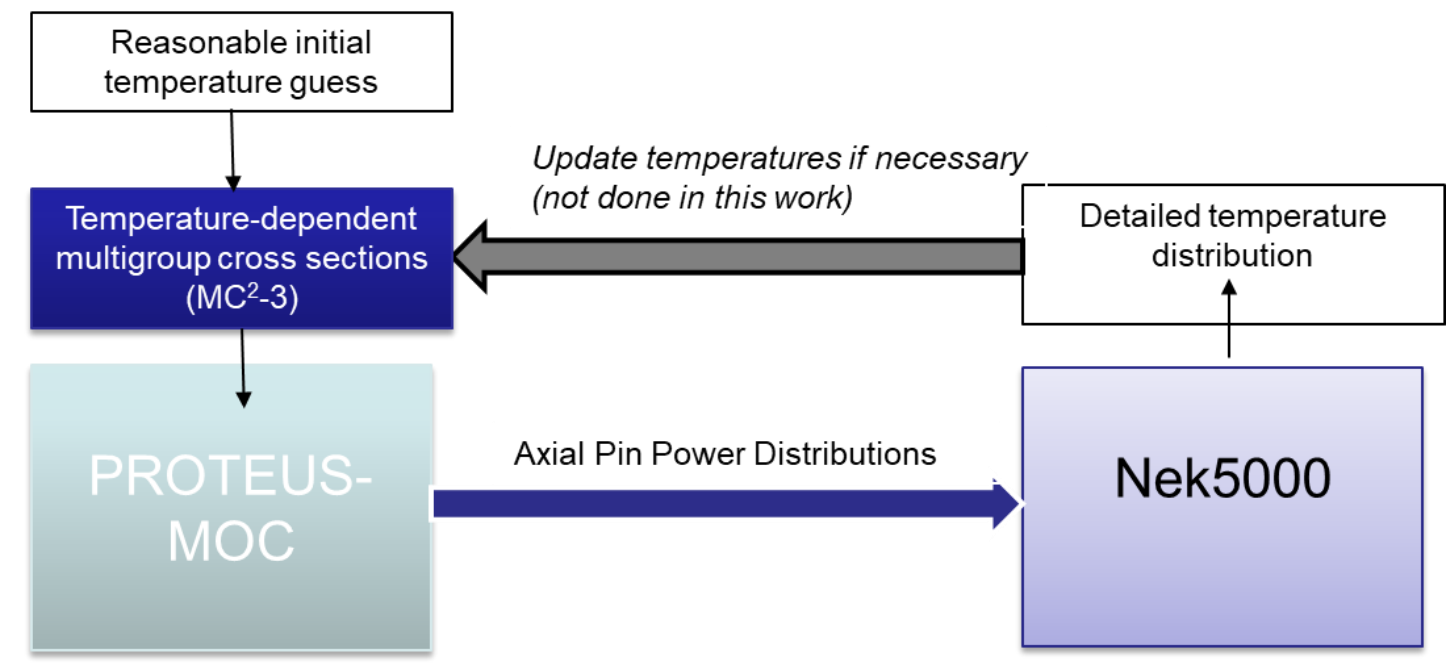

Figure 2.3.3 Schematic of offline coupling using components of the SHARP toolkit.

The offline coupling was performed one-way: initial calculation with PROTEUS-MOCEX, spatial distribution of power density given to Nek5000, and finally Nek5000 calculates temperature distribution. Ideally an iterative procedure would be implemented where neutronics would update the material cross sections based on temperature, and produce a new power density for Nek5000, but this would double the computational requirements and usually is not necessary due to only minor dependence of power distribution on temperature feedback for fast reactors.

Local machines and small clusters are used to perform pre-processing steps such as finite element mesh generation, multigroup cross section preparation, and testing of input files. To perform more detailed testing, debugging, convergence and sensitivity studies, as well as small production runs, an allocation at Argonne's Laboratory Computing Resource Center (LCRC) Bebop machine was utilized. The Bebop cluster has 664 Broadwall nodes with 36 cores/node and $128 \mathrm{~GB} /$ node, plus 352 Intel Xeon Phi (Knights Landing) nodes with 64 cores/node and 96 GB. Roughly $150 \mathrm{~K}-300 \mathrm{~K}$ core hours are available per quarter, depending on user needs.

While not needed in general for the HCF calculations (due to computational advancements in the Nek5000 single assembly model and lack of wire wrap), large production simulations are needed for the zooming calculations discussed later. An Advanced Scientific Computing Research (ASCR) Leadership Computing Challenge (ALCC) proposal was written and awarded 880,000 node-hours of compute time at the Argonne Leadership Computing Facility (ALCF) Theta machine. ALCF's Theta has 4,392 Intel Xeon Phi (Knights Landing) nodes with 64 cores and 16 GB of MCDRAM, and 192 GB DDR4 memory. The ALCC award runs from July 1, 2019 through June 30, 2020.

The required high-fidelity calculations for the HCF evaluations are summarized in Table 2.3.1. The PROTEUS and Nek5000 columns in this table indicate the required number of highfidelity assembly calculations by PROTEUS and Nek5000 codes. A nominal condition 
calculation is required to understand the power and temperature distribution without accounting for any uncertainties. The peak temperatures obtained from each perturbed calculation is then compared with nominal condition temperatures to evaluate the hot channel factors.

Table 2.3.1. Required high-fidelity calculations for HCF evaluations

\begin{tabular}{|l|c|c|}
\hline \multicolumn{1}{|c|}{ HCF } & PROTEUS & Nek5000 \\
\hline Nominal Condition & 1 & 1 \\
\hline Cladding Thickness & 1 & 2 \\
\hline Fissile Maldistribution & 30 & 30 \\
\hline Coolant Specific Heat & - & 1 \\
\hline Gap Conductance (Bounding Case) & 1 & 2 \\
\hline
\end{tabular}

Only the fissile maldistribution case is analyzed using a stochastic sampling technique. Ideally the cladding thickness case would also have been treated this way, however, meshing stochastically variable pins is extremely time consuming, so instead the pins were changed uniformly by the maximum cladding tolerance value to decrease the cladding thickness to its thinnest size. In Nek5000, both increased and decreased cladding thicknesses were analyzed to understand the dual impacts of changing subchannel flow area as well as changing cladding thickness. The coolant specific heat calculation is necessary only in Nek5000. The gap conductance bounding case is performed for burned fuel composition with a fully closed gap. Additional information on the formation of joint-oxide gain (JOG) is necessary to fully evaluate HCF for this case.

\subsection{Neutronics Modeling Strategy}

The PROTEUS-MOCEX transport solver was employed to solve each of the simulations shown in Table 2.3.1. Each simulation represents a single 3D LFR fuel assembly in fully heterogeneous detail, with reflective boundary conditions placed on the exterior radial boundaries and void boundary conditions placed on the top and bottom boundaries. PROTEUSMOCEX was chosen due to its efficiency on relatively small but heterogeneous geometries, especially those including streaming effects in near-void regions (i.e. the annular fuel pellet). PROTEUS-MOCEX is based on a method of characteristics formulation in the radial (X-Y) plane and discontinuous Galerkin finite element formulation in the axial (Z) dimension.

\subsubsection{Cross Section Generation}

Multigroup cross sections were generated using the $\mathrm{MC}^{2}-3$ code [Lee 2012] which specializes in fast spectrum reactors. Heterogeneity effects were accounted for through a two-step procedure using $\mathrm{MC}^{2}-3$ in conjunction with TWODANT [Alcouffe 1984]. The workflow used for MC ${ }^{2}-$ 
3/TWODANT is shown in Figure 2.4.1 and is based on previous experience generating multigroup cross sections for highly heterogeneous geometries [Hader 2014], [Jarrett 2016]. The assembly is converted into a 1-D geometry by homogenizing materials within each axial zone (active fuel, thermal insulator, etc). Each assembly axial zone is simulated as an infinite medium homogeneous mixture in $\mathrm{MC}^{2}-3$ to yield 1041-group cross sections. A 1041-group 1-D transport simulation is then performed in TWODANT to calculate the axial leakage in each axial zone. The resulting 1041-group region dependent fluxes are used to collapse the 1041-group cross sections for the non-fuel axial zones into broad group cross sections. For axial zones containing fuel, new 1041-group cross sections including pin radial heterogeneity effects were generated by converting the 127-pin geometry into 1-D cylindrical geometry in $\mathrm{MC}^{2}-3$ while preserving volume fractions of each material to model the explicit fuel, cladding and coolant materials. Such a conversion is depicted in Figure 2.4.2 (rings are not to scale or correct in number). The rings represent materials like fuel, cladding, coolant, etc. The resulting fuel zone 1041-group cross sections were then collapsed with the TWODANT 1041-group fluxes to yield broad group (33group) cross sections with both local and global heterogeneity effects.

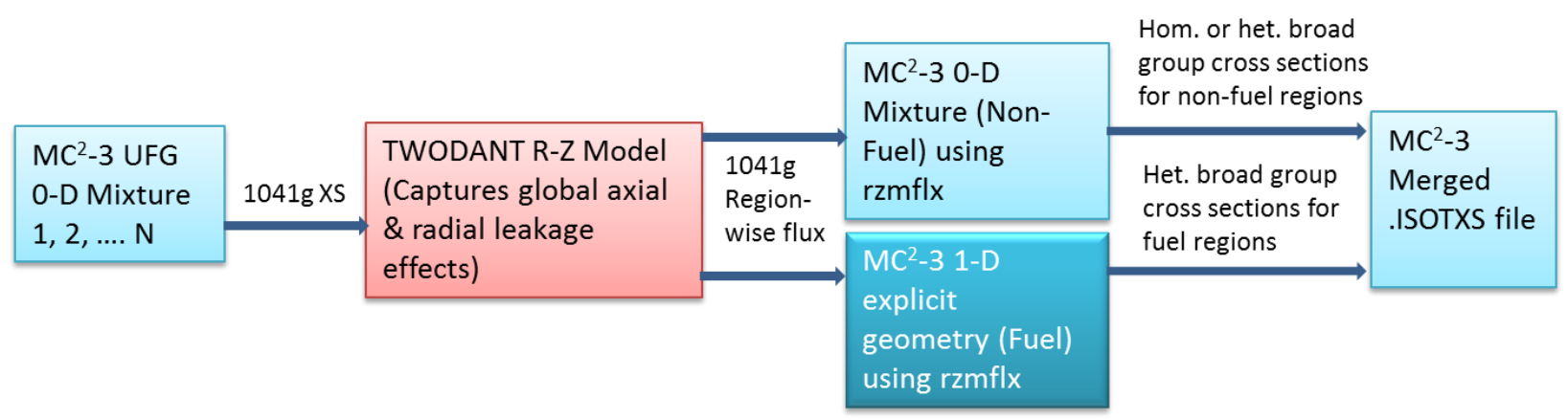

Figure 2.4.1 Cross section generation procedure for heterogeneous 3D pin configurations

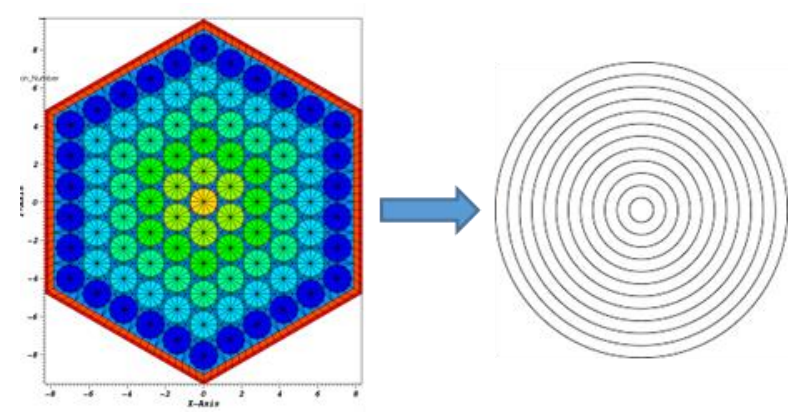

Figure 2.4.2 Conversion of assembly into 1-D cylinder geometry (not to scale) 


\subsubsection{Mesh Generation}

The PROTEUS MeshTools code was chosen to generate the PROTEUS meshes as it easily incorporates repeated pin cell features in a hexagonal geometry, as well as assembly ducts. Meshes of varying degrees of fidelity were generated in order to perform mesh convergence studies. The azimuthal intervals (A) and radial intervals $(\mathrm{R})$ for the fuel pins can be varied independently of each other, and the number of axial planes can also be varied. For PROTEUSMOCEX, a 2D finite element mesh is required, along with the axial plane discretization provided separately.

The nominal mesh is used for all simulations except the cladding thickness perturbation. The nominal cross section file is used for all simulations except the burned fuel simulation needed for the gap conductance study. While the material assignments change for the fissile maldistribution cases, they are based on the nominal cross section data.

\subsubsection{Neutronics Discussion of Nominal Condition}

Convergence studies in mesh and angular cubature were performed to ensure appropriate selection of discretization parameters in the neutronics model. The energy group structure was held constant at 33 energy groups. The full assembly problem showed little to no dependence on mesh fidelity. A mesh with 10,980 elements in the X-Y plane and 48 axial planes was used in the final calculations (see Figure 2.4.3). The eigenvalue was strongly dependent on the angular cubature due to streaming regions in the annular fuel pellet. The cubature selected was LegendreTchebychev L7T17 (288 angles on the unit sphere) which exhibits a good balance of accuracy and computational cost. The 3D nominal condition fuel assembly eigenvalue was computed as 1.17701 . 

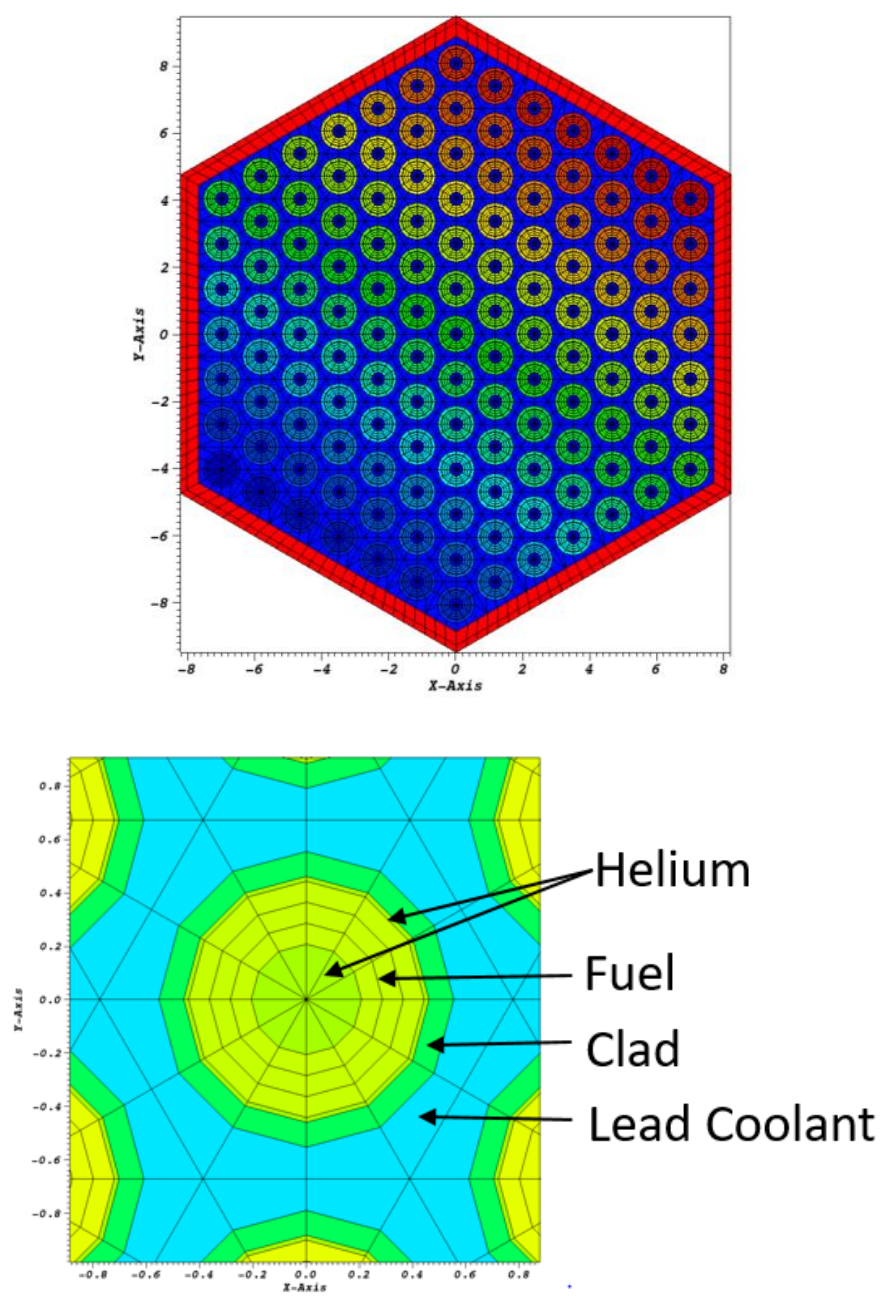

Figure 2.4.3 Top view of LFR neutronics mesh (left) and zoomed in (right)

Code-to-code verification of eigenvalues was performed for various LFR models to ensure the cross sections and geometry were generated correctly (Table 2.4.2). The MCNP6 continuous energy code was used for comparison to $\mathrm{MC}^{2}-3$ (when applicable) and PROTEUS-MOC. Three infinite medium cases were run (LFR MOX fuel, LFR pin cell, and LFR active fuel zone of assembly) and showed good agreement with both $\mathrm{MC}^{2}-3$ and PROTEUS-MOC eigenvalues. The 2D LFR pin cell also showed good agreement with both codes. Due to geometry limitations, geometrically complex cases are not performed in $\mathrm{MC}^{2}-3$. The 2D LFR assembly compared well to PROTEUS-MOC, and while the 3D LFR assembly (the case of relevance to the hot channel factors) showed larger errors, the agreement is still reasonable and could potentially be reduced with additional energy groups in PROTEUS-MOC. Notably, the pin power distribution in the LFR assembly center pin (pin 1) are compared in MCNP and PROTEUS-MOC and match within $1.5 \%$ error (see Figure 2.4.4). The pin power distribution is the only data transferred to Nek5000. 
The LFR assembly model in MC M $^{2}$ /PROTEUS therefore matches well against the completely independent model in continuous energy MCNP.

Table 2.4.2. Code-to-code eigenvalue verification of LFR-based problems

\begin{tabular}{|l|c|c|c|}
\hline \multicolumn{1}{|c|}{ LFR Case } & $\begin{array}{c}\text { MCNP6 CE } \\
\text { (pcm uncert.) }\end{array}$ & $\begin{array}{c}\text { MC'-3 1041g } \\
- \text { MCNP (pcm) }\end{array}$ & $\begin{array}{c}\text { PROTEUS-MOC 33g } \\
\text { - MCNP (pcm) }\end{array}$ \\
\hline $\begin{array}{l}\text { Infinite medium } \\
\text { (homogenized LFR MOX fuel) }\end{array}$ & 1.65235 (15) & 177 & 180 \\
\hline $\begin{array}{l}\text { Infinite medium } \\
\text { (homogenized LFR pin cell) }\end{array}$ & 1.36242 (11) & 77 & 184 \\
\hline $\begin{array}{l}\text { Infinite medium } \\
\text { (homogenized LFR active fuel zone } \\
\text { of assembly) }\end{array}$ & $1.28613(11)$ & 13 & 188 \\
\hline 2D LFR pin cell & $1.36625(12)$ & 65 & 214 \\
\hline $\begin{array}{l}\text { 2D LFR assembly } \\
\text { (active core slice) }\end{array}$ & $1.29446(3)$ & - & 413 \\
\hline $\begin{array}{l}\text { 3D LFR assembly } \\
\text { (“nominal condition”) }\end{array}$ & $1.17288(19)$ & - & \\
\hline
\end{tabular}




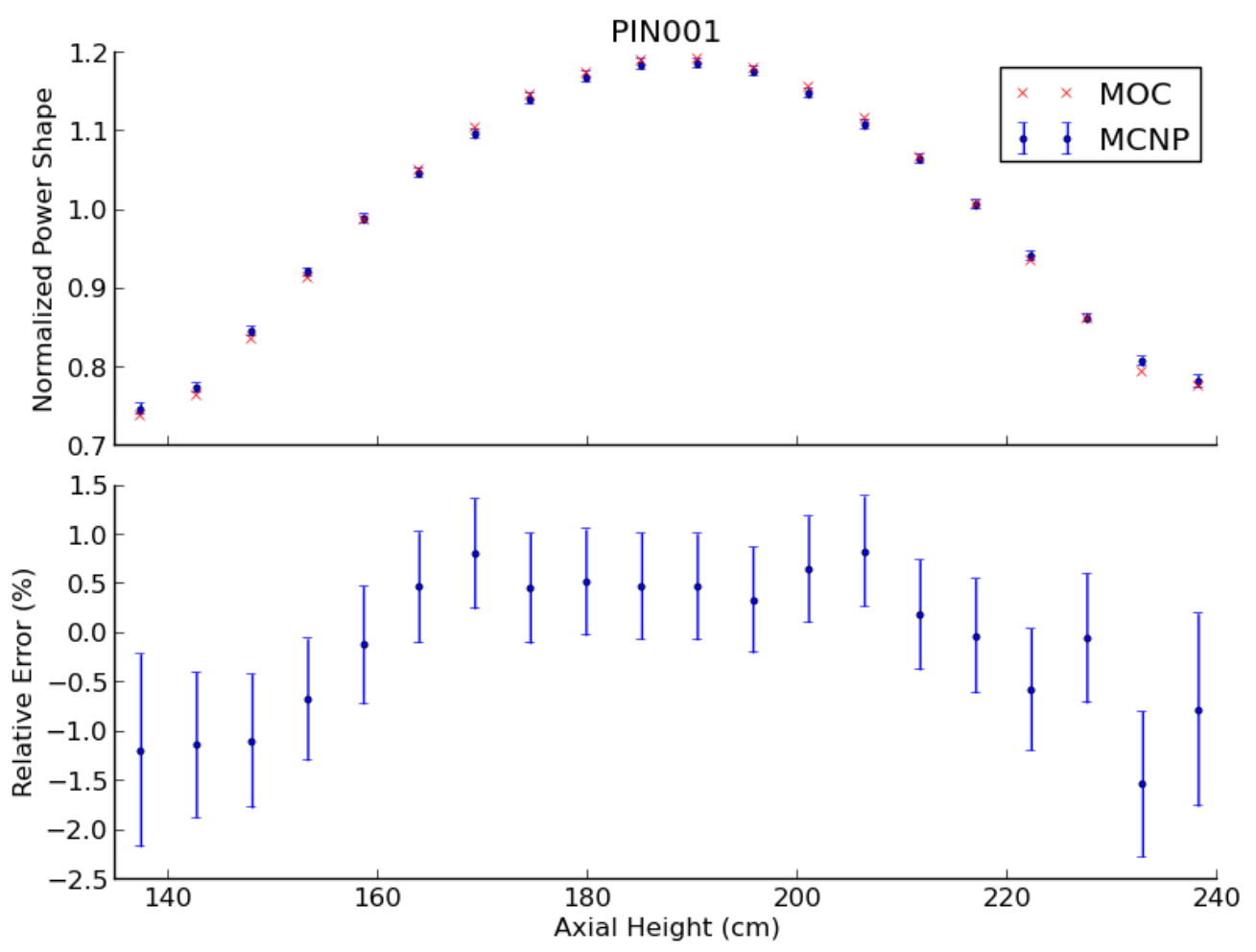

Figure 2.4.4 Code-to-code verification of center pin power shape

The nominal condition calculation is simply the 3D LFR assembly case from Table 2.4.1. The power in the assembly was normalized to $3.7 \mathrm{MW}$. The pin power distribution for the center pin is shown in Figure 2.4.4. Three axial slices taken at the core bottom, middle, and top depict the radial power distribution in the assembly (Figure 2.4.5). The same color scale is used in all plots and shows that the power level is highest at mid-core, and slightly higher at the top of the core than at the bottom of the core (shifted cosine shape). This matches the pin power distribution in Figure 2.4.6. The pin powers exhibit a nearly flat radial power distribution at all axial levels. This is expected because of reflected boundary conditions placed on the exterior of the assembly. There is also a nearly constant axial shape and integrated pin power for all 127 pins. The maximum and minimum integrated fuel pin powers are $28.76 \mathrm{~kW}$ (outside corner pins) and 28.68 $\mathrm{kW}$ (inner rings), i.e. $<0.2 \%$ difference. The maximum and minimum cladding powers are 100.6 $\mathrm{W}$ (outside corner pins) and $98.9 \mathrm{~W}$ (inner rings). The cladding power is negligible in the fuel assembly compared to the fission power generated in the fuel zones. However it is tallied separately for Nek5000 due to the fuel-cladding gap. No gamma transport was applied in these cases, so gamma particles are assumed to deposit heat at their source site. It should be noted that the total heat generation of the 127 fuel pins and cladding is $3.6577 \mathrm{MW}$, compared to $3.7 \mathrm{MW}$ assembly power. The missing $1.1 \%$ of total assembly power is contained within duct walls or in regions outside the active core and is ignored in Nek5000. Power outside the active core is 
subsequently ignored in Nek5000 as it does not significantly contribute to the coolant, cladding, or fuel temperatures in the active core.
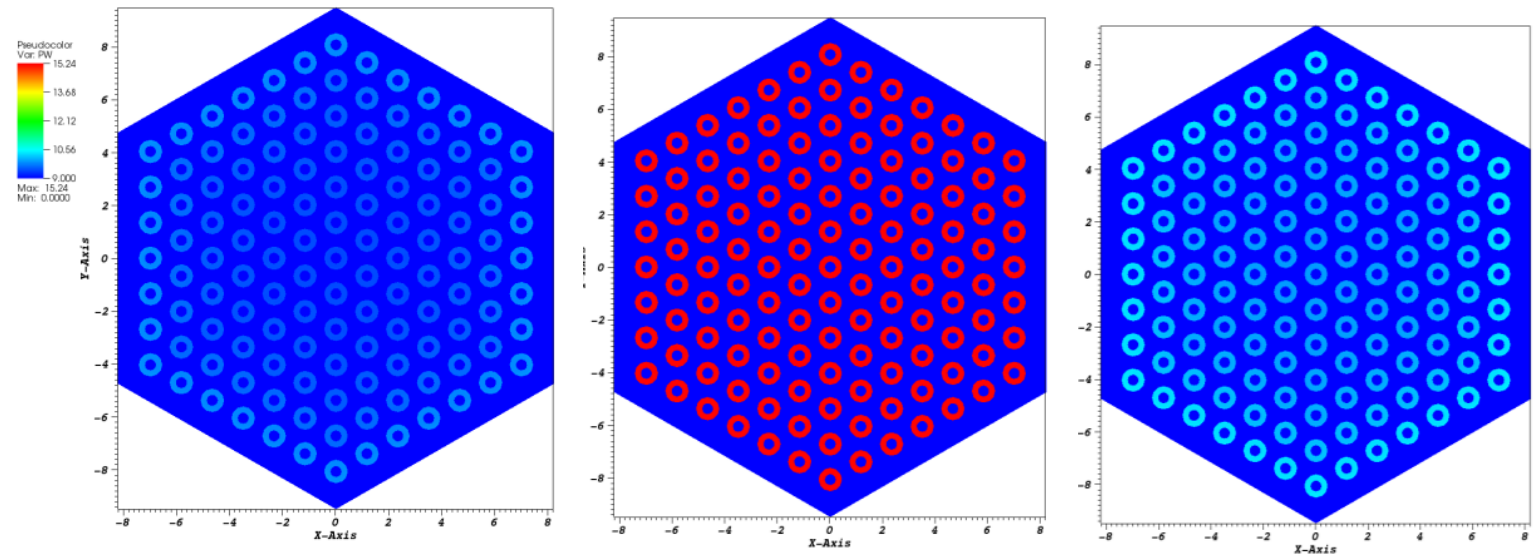

Figure 2.4.5 Left to right: Power distributions shown at bottom $(\mathrm{z}=135 \mathrm{~cm})$, middle $(\mathrm{z}=\mathbf{1 8 7} \mathrm{cm})$ and top $(\mathrm{z}=\mathbf{2 4 0} \mathrm{cm})$ of core

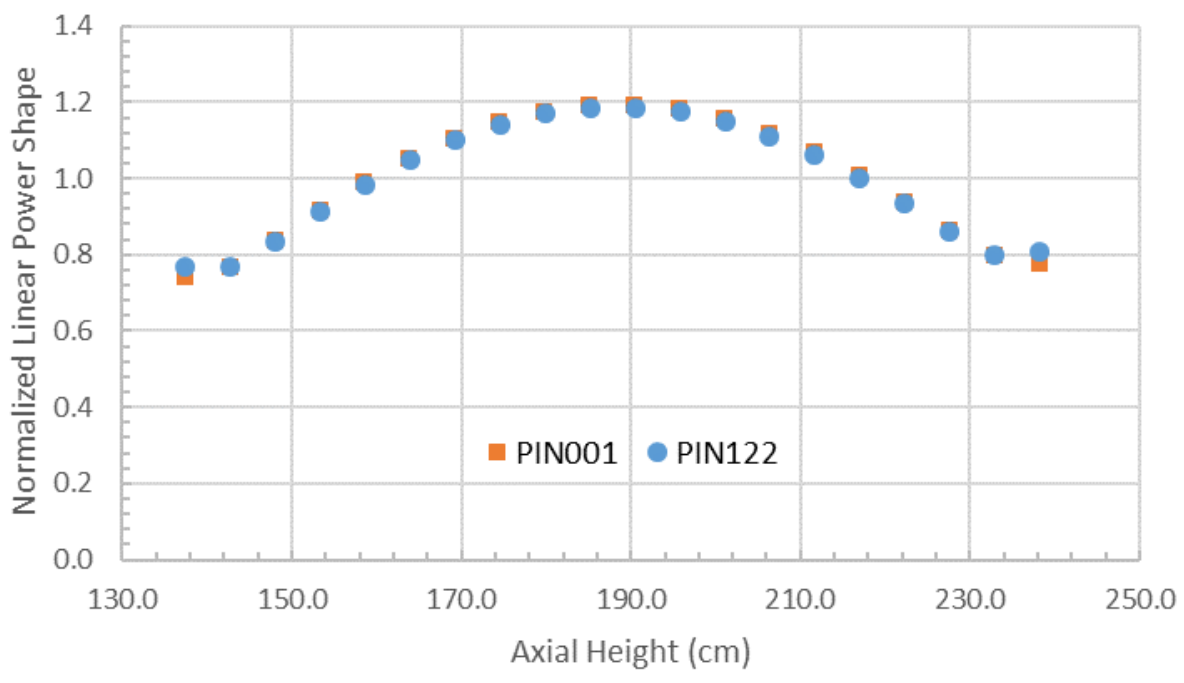

Figure 2.4.6 Axial power shapes in fuel zone of pin 1 (center) and pin 122 (outside corner)

To summarize the nominal condition neutronics analysis, the pin and cladding power shapes are nearly identical. The same axial power shape can be used for both all fuel and cladding zones. A uniform power distribution across pins is also valid since the maximum and minimum integrated pin powers differs by only $0.2 \%$. The nominal condition calculation requires 12 
minutes of wall clock time on 96 Broadwell nodes (36 cores/node) or 691 core-hours. The MCNP verification calculation required 20 hours of wall clock time on 32 processors of Intel Xeon X5560 or 640 core-hours.

\subsection{Thermal Fluid Modeling Strategy}

In the Nek5000 CFD simulation, the cladding, fuel, helium gap and hollow are modeled explicitly. A new mesh script was developed to mesh these domains separately. Models for calculating the impact of the cladding thickness perturbation or other geometric perturbation can thus be easily built by taking advantage of the meshing script.

\subsubsection{Lateral Velocity Considerations}

URANS simulations are performed with k- $\omega$ turbulence model for the LFR fuel assembly. Figure 2.5.1 shows the lateral velocity on different elevations. The simulation indicates there exists strong lateral velocity in the vicinity of the duct. The assembly has a relatively small gap between the outer ring of pins and the duct wall, which provides relatively strong shear stress. The flow near the duct swirls around the outmost layer pin when moving in the stream-wise direction. The magnitude of the strongest lateral velocity can reach $30 \%$ of the mean streamwise velocity. It can also be observed that the lateral flow needs a certain distance to fully develop. On the first two elevations near inlet, the lateral velocity is not very obvious. The lateral flow becomes fully developed after it flows past half of the domain. The strong lateral velocity only appears near the duct while it stays small in the center of the assembly.

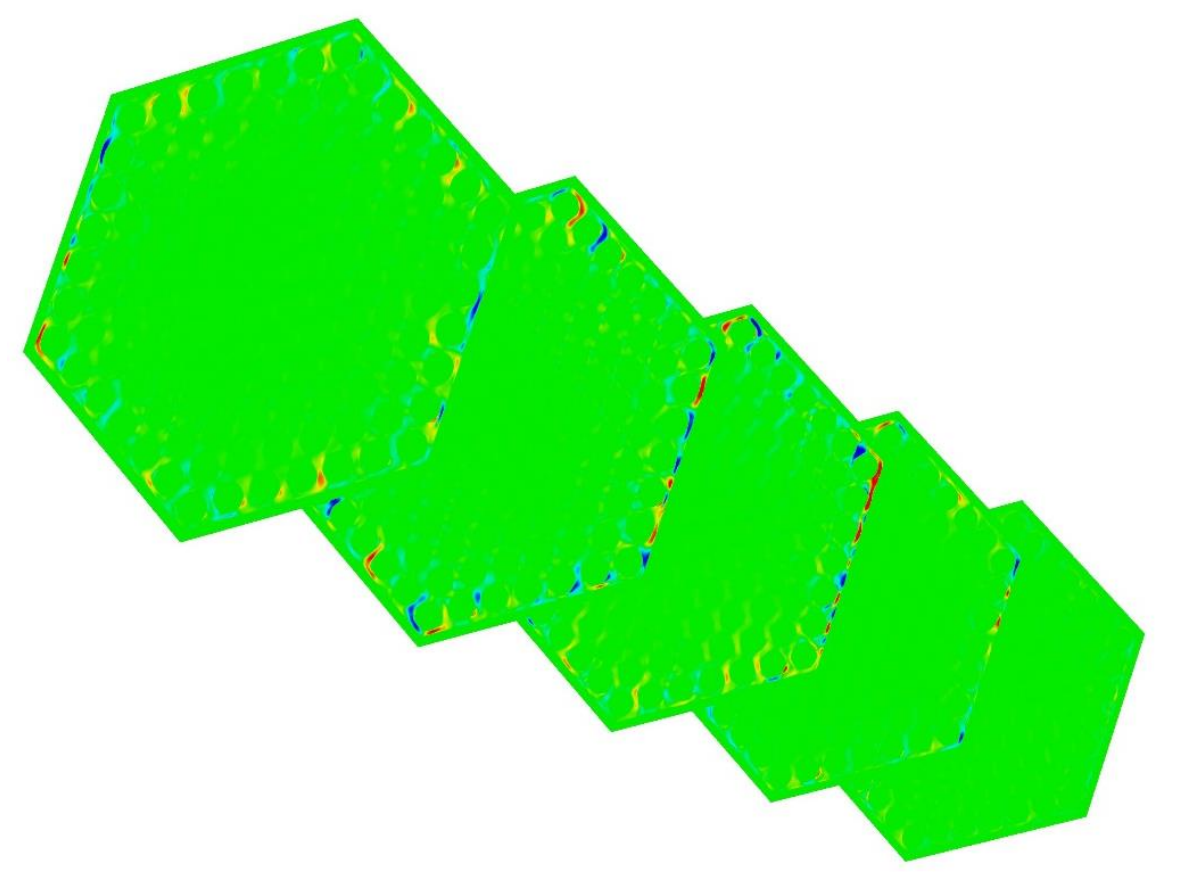

Figure 2.5.1 Lateral velocity on different elevations of the LFR assembly 
Figure 2.5.2 shows the transient lateral velocity near the corner of the LFR fuel assembly, i.e. how the lateral velocity changes over time. The fluid in the corner of the assembly oscillates in direction within the plane, which leads to similar time-averaged lateral velocity predicted from a RANS simulation. However, it is always preferable to capture these transient flow behaviors with a URANS model.

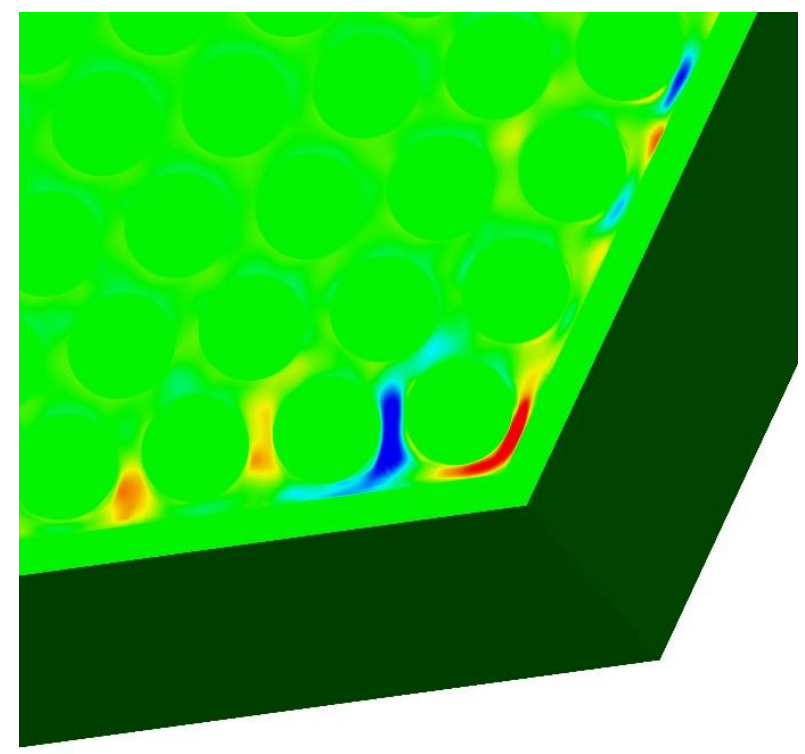

$0.5 \mathrm{~s}$

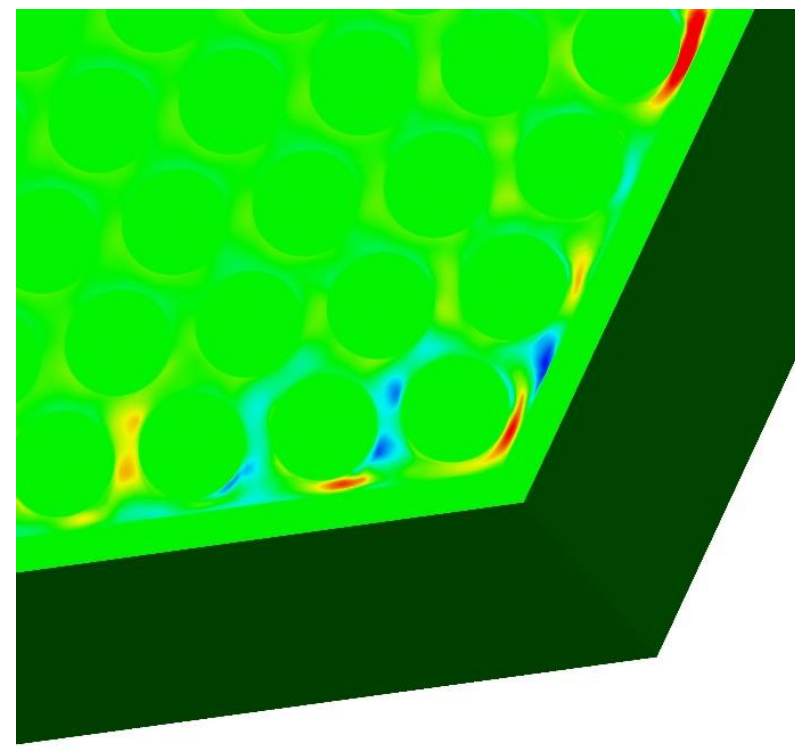

$1.5 \mathrm{~s}$

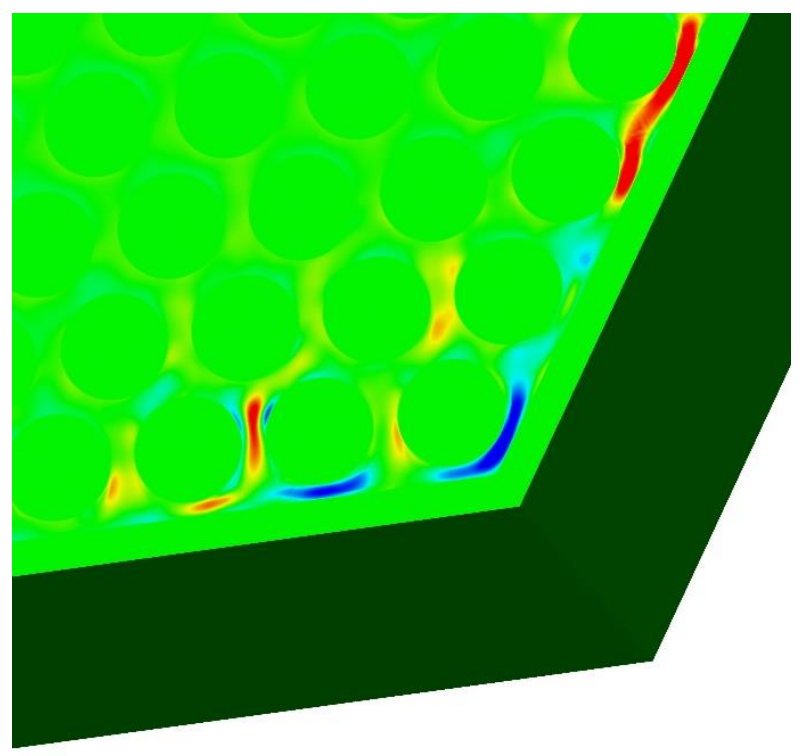

$1 \mathrm{~s}$

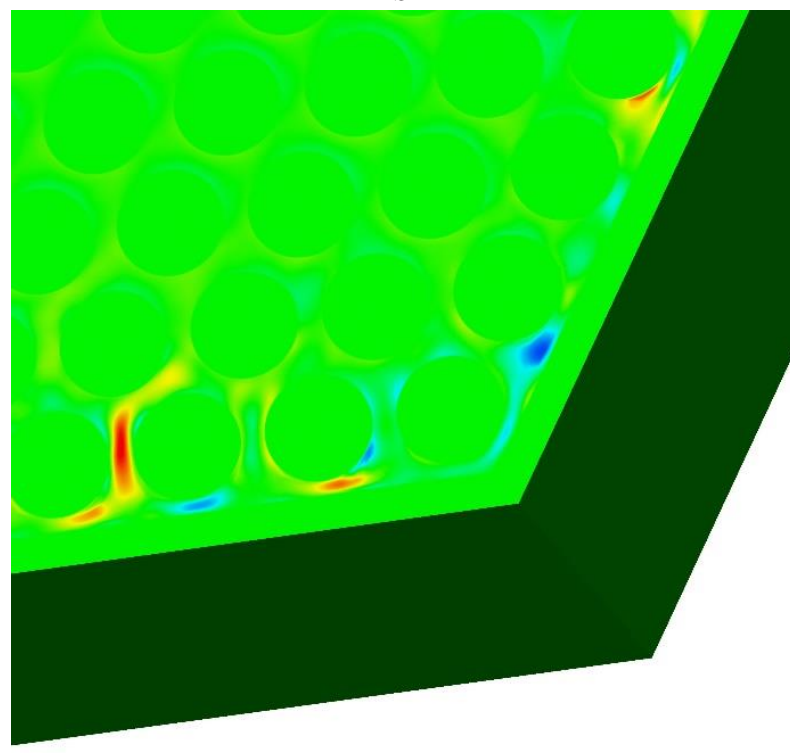

$2 \mathrm{~s}$

Figure 2.5.2 Transient lateral velocity near the corner of the LFR assembly 


\subsubsection{Turbulent Prandtl Number Consideration}

Heavy metal fluid (lead) is different from sodium fluid. In general, the Prandtl number of heavy metal fluid is two orders of magnitude lower than that of sodium fluid. Due to the low Prandtl number of lead, the thermal boundary layer in the temperature field is much thicker than the viscous boundary layer in the velocity field. Existing engineering turbulence models all use the Reynolds analogy for coupling temperature and velocity fields, which is not valid for Heavy Lead Metal (HLM) flow. Therefore, unlike for sodium flow, the selection of an appropriate turbulent Prandtl number, $\operatorname{Pr}_{t}$, is crucial for lead fluid simulation. The conventional choice of $\operatorname{Pr}_{t}$ as a constant value of $0: 85$ to $0: 9$ is not valid for low-Prandtl number fluid flow. Both experimental and theoretical studies [Reynolds 1975][Cheng 2004] available in the open literature suggest a turbulent Prandtl number larger than 1.0 for liquid metal flows.

In the past, extensive studies were carried out to derive appropriate expressions for turbulent Prandtl number $\mathrm{Pr}_{t}$ for low-Prandtl fluid. In general, two types of turbulent Prandtl number model are developed. The first type specifies $\operatorname{Pr}_{t}$ as a global value depending on the bulk flow parameters. The second type takes $\operatorname{Pr}_{t}$ as a local varying value depending on the local flow parameters such as the local eddy diffusivity. These correlations listed in Table 2.5.1 shows both type of $\operatorname{Pr}_{t}$ models and indicate the relationships between $\operatorname{Pr}_{t}$ and Reynolds number, Peclet number (Pe) and Prandtl number (Pr) in each model. Different models have been suggested for $\mathrm{Pr}_{\mathrm{t}}$ estimation based on different data sources, as shown in the figure. The final column of the table is the calculated $\operatorname{Pr}_{\mathrm{t}}$ based on the LFR-specific design data in Table 2.1.1.

Table 2.5.1. Correlations for turbulent Prandtl Number

\begin{tabular}{|c|c|c|c|}
\hline Reference & Data Source & Correlation & $\operatorname{Pr}_{t}$ \\
\hline $\begin{array}{c}\text { [MYONG } \\
1989]\end{array}$ & $\begin{array}{l}\text { RANS } \\
\text { Simulations } \\
\text { (pipe) }\end{array}$ & $P r_{t}=0.75+\frac{1.63}{\ln (1+\operatorname{Pr} / 0.0015)}$ & 1.45 \\
\hline $\begin{array}{l}\text { [X. Cheng } \\
\text { 2006][1] }\end{array}$ & $\begin{array}{c}\text { RANS } \\
\text { Simulations } \\
\text { (sub-channel in } \\
\text { wide lattice) }\end{array}$ & $\operatorname{Pr}_{t}=\left\{\begin{array}{cc}1.5 & P e \leq 2000 \\
2.5-0.0005 P e & 2000 \leq P e \leq 3000 \\
1.0 & P e \geq 3000\end{array}\right.$ & 1.5 \\
\hline $\begin{array}{l}\text { [X. Cheng } \\
\text { 2006][2] }\end{array}$ & $\begin{array}{l}\text { RANS } \\
\text { Simulations } \\
\text { (sub-channel in } \\
\text { wide lattice) }\end{array}$ & $\begin{array}{c}P r_{t}\left\{\begin{array}{cc}4.12 & P e \leq 1000 \\
\frac{0.01 P e}{\left[0.018 P e^{0.8}-(7-A)\right]^{1.25}} 1000 \leq P e \leq 6000\end{array}\right. \\
\mathrm{A}=\left\{\begin{array}{cc}4.5 & P e \leq 1000 \\
5.4-0.0009 P e & 1000 \leq P e \leq 2000 \\
3.6 & P e \geq 2000\end{array}\right.\end{array}$ & 4.12 \\
\hline $\begin{array}{l}\text { [A. } \\
\text { Reynolds } \\
1975]\end{array}$ & $\begin{array}{l}\text { Analytical and } \\
\text { empirical } \\
\text { approaches }\end{array}$ & $P r_{t}=\left(1+100 P e^{-0.5}\right)\left(\frac{1}{1+120 R e^{-0.5}}-0.15\right)$ & 2.4 \\
\hline
\end{tabular}




\begin{tabular}{|c|c|c|c|}
\hline $\begin{array}{l}\text { [Aoki } \\
1963]\end{array}$ & $\begin{array}{l}\text { Analytical and } \\
\text { empirical } \\
\text { approaches }\end{array}$ & $\begin{array}{l}\operatorname{Pr}_{t}^{-1}=0.014 R e^{0.45} \operatorname{Pr}^{0.2} \times \\
{\left[1-\exp \left(\frac{-1}{0.014 R e^{0.45} \operatorname{Pr}^{0.2}}\right)\right]}\end{array}$ & 1.77 \\
\hline $\begin{array}{c}\text { [Jischa } \\
1979]\end{array}$ & $\begin{array}{l}\text { Analytical and } \\
\text { empirical } \\
\text { approaches }\end{array}$ & $P r_{t}=0.9+\frac{182.4}{\operatorname{PrRe} e^{0.888}}$ & 1.77 \\
\hline $\begin{array}{l}\text { [Kays } \\
1994]\end{array}$ & $\begin{array}{l}\text { Experiments } \\
\text { (Tube and Duct) }\end{array}$ & $\begin{array}{l}P r_{t}=0.85+\frac{0.7}{P e_{t}} \\
P r_{t}=\frac{v_{t}}{v} \operatorname{Pr}=\frac{v_{t}}{\alpha}\end{array}$ & - \\
\hline
\end{tabular}

Figure 2.5.3 compares the $\mathrm{Pr}_{t}$ obtained with these models for Peclet number up to 4800 at Prandtl number 0.0136 (Westinghouse LFR design). The $\operatorname{Pr}_{t}$ generally decreases with the increase of Pe. As Pe becomes larger than 2000, the predicted $\mathrm{Pr}_{t}$ for each model tends to approach an asymptotic value between 0.5 and 2.5 .

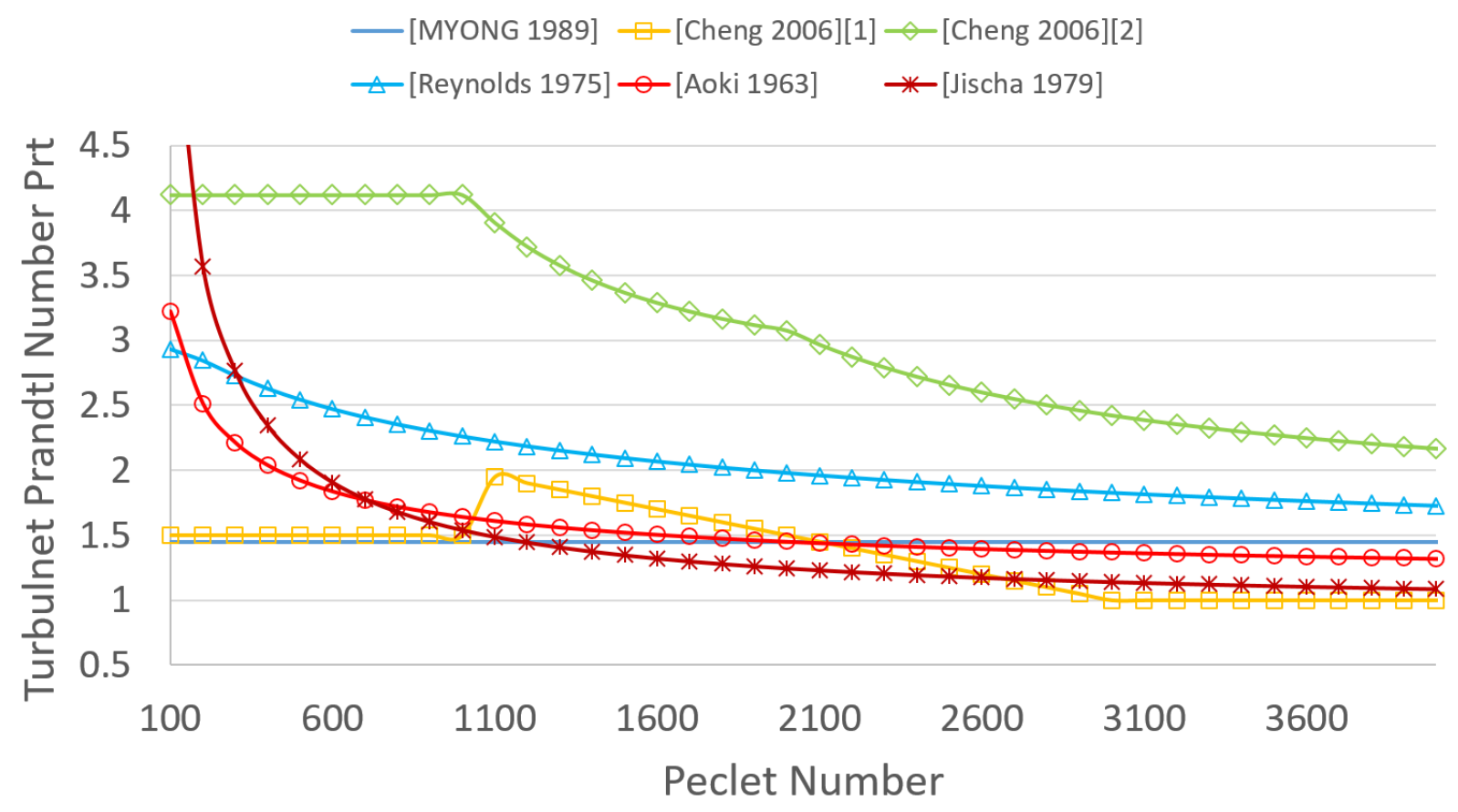

Figure 2.5.3 $\mathrm{Pr}_{\mathrm{t}}$ estimation with different models $(\mathrm{Pr}=0.0136)$

A large scattering exists in the predicted values of $\operatorname{Pr}_{t}$ obtained by different models especially in the range of $\mathrm{Pe}<1000$, which is due to lack of reliable experimental data on turbulent heat transfer in liquid metal flows. Advanced high-fidelity numerical approaches such as Direct Numerical 
Simulation (DNS) and Large Eddy Simulation (LES) are applied to provide detailed insight into the physics of the flow and the associated heat transfer in rod bundle with liquid metal flows [Lai 2019]. Very limited data are reported for liquid metal flows heat transfer in rod bundle with LES simulation. LES simulations were performed here to provide a valuable reference to which RANS models can be compared and calibrated. RANS simulations with fixed values of $\operatorname{Pr}_{t}$ were compared to the reference LES simulation to determine the appropriate choice of the $\operatorname{Pr}_{t}$ when using a RANS approach.

For the study on best choice of $\operatorname{Pr}_{t}$ with RANS approach, the computational domain consists of two sub-channels connected by a narrow gap (Figure 2.5.4), based on the LFR geometry. The boundary conditions include two couples of periodic boundaries and non-slip walls. In the present case, the computational length has been chosen to be the full length of the active core with inlet velocity boundary and pressure outlet boundary to incorporate the power distribution from the neutronics code.
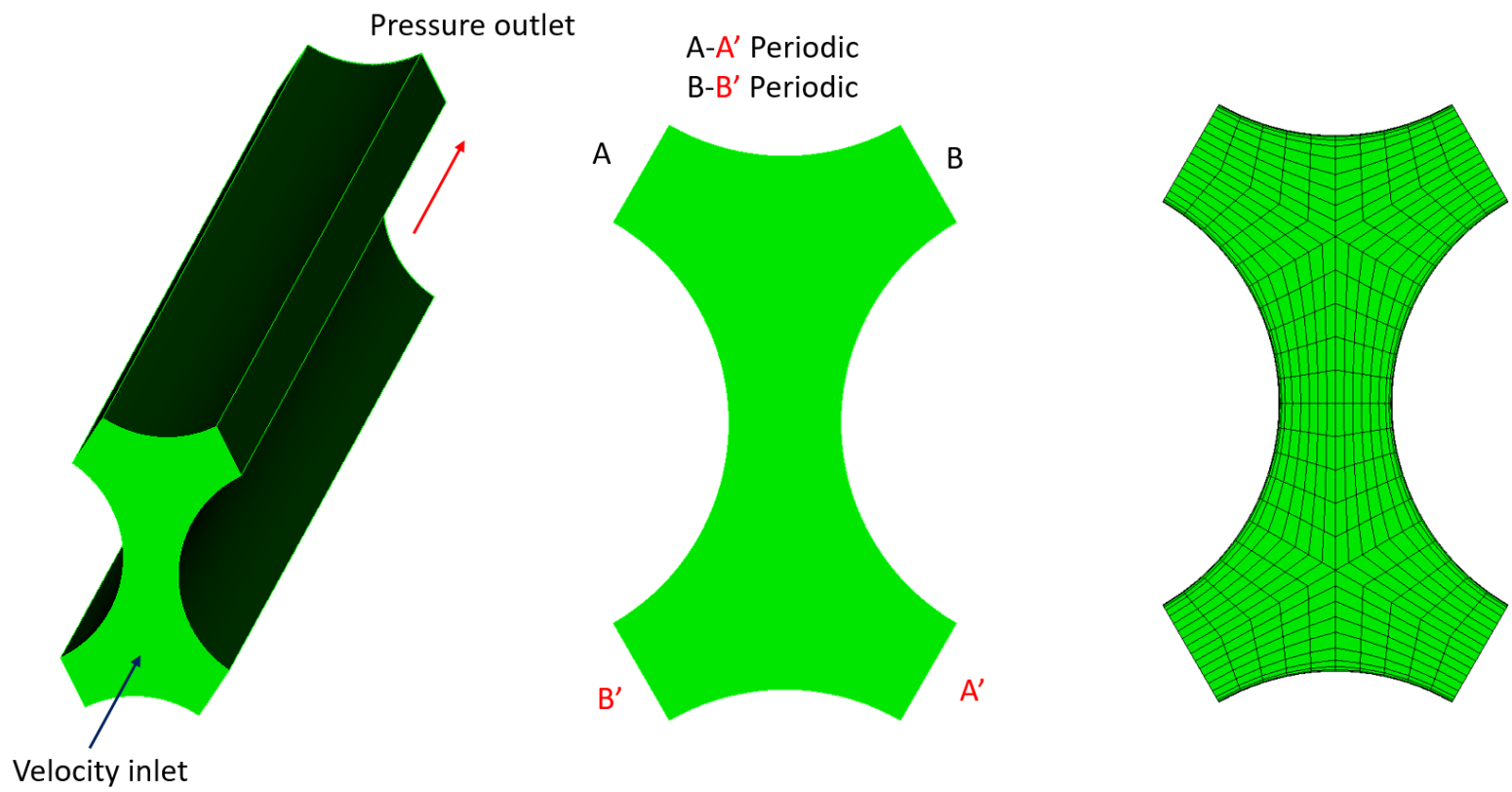

Figure 2.5.4 Computational domain, Boundary conditions and Mesh for LES

Heat transfer performance can be characterized by the Nusselt number $(\mathrm{Nu})$. For liquid metals, several authors proposed heat transfer correlations (Table 2.5.2) which only depend on $\mathrm{Pe}=\mathrm{RePr}$, thus attributing the same importance to Re and Pr. Figure 2.5.5 shows the Nusselt number prediction with different correlations, LES and URANS simulation results. For turbulent flow, the correlations proposed by Cheng and Tak are in good agreement with the simulation results, especially for $\mathrm{Pe}<1000$. LES and URANS model with $\mathrm{Pr}_{t}=1.5$ predicts similar Nusselt number. 
Table 2.5.2. Correlations for Nusselt Number

\begin{tabular}{|c|c|c|}
\hline Reference & Data Source & Correlation \\
\hline $\begin{array}{c}\text { [Lubarsky } \\
1995]\end{array}$ & Experiments & $N u=0.625 P e^{0.4}=0.625 \mathrm{Re}^{0.4} \mathrm{Pr}^{0.4}$ \\
\hline $\begin{array}{c}\text { [X.Cheng } \\
\text { 2006][1] }\end{array}$ & $\begin{array}{c}\text { RANS } \\
\text { Simulations } \\
\text { (sub-channel in } \\
\text { wide lattice) }\end{array}$ & $N u=A+0.0155\left(\frac{P e}{P r_{t}}\right)^{0.86}$ \\
\hline & $\begin{array}{c}\text { RANS } \\
\text { [X.Cheng } \\
\text { 2006][2] }\end{array}$ & $\begin{array}{c}\text { Simulations } \\
\text { (sub-channel in } \\
\text { wide lattice) }\end{array}$ \\
\hline
\end{tabular}

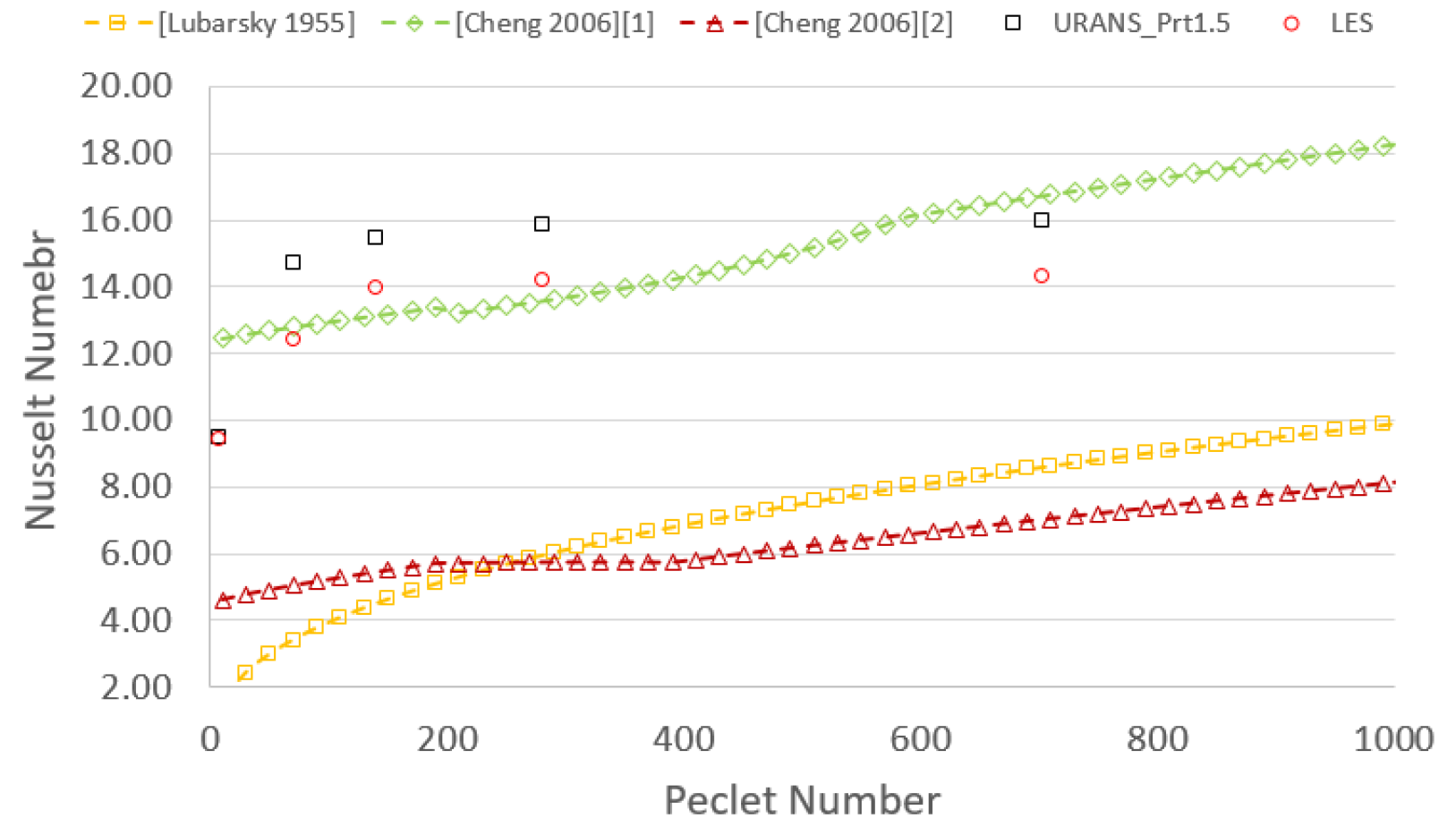

Figure 2.5.5 Nusselt number prediction with different models

The quantitative comparison of temperature distribution between rods are made between URANS models with varying turbulent Prandtl number (Figure 2.5.6). The results indicate that 
the temperature distribution is sensitive to turbulent Prandtl number. The estimation of global Nusselt number with different turbulent Prandtl number (0.9 2.5) shows that a higher turbulent Prandtl number can reduce the predicted Nusselt number by up to $38.4 \%$. LES results predict the lower temperature profile in the center of the gap. That is because that LES can simulate the flow oscillation in the gap while URANS models fails to capture that. As shown in Figure 2.5.7, even in a wide lattice $(\mathrm{P} / \mathrm{D}=1.242)$ configuration, the flow oscillation which enhances the mass and heat transfer between sub-channels still exists. With the increase of Reynolds number, the flow oscillation become more chaotic. The local turbulent Prandtl model seems to be a promising approach on calibrating the turbulence model. However, the local turbulent Prandtl model suggested by [Kays 1994] obtains little improvement on temperature distribution prediction. It is likely due to the data source of the model, which comes from the experimental data in tube and duct rather than rod bundles. 
O Prt $0.9 \square$ Prt $1.5 \Delta$ Prt $2 \diamond$ Prt local $\bullet$ LES

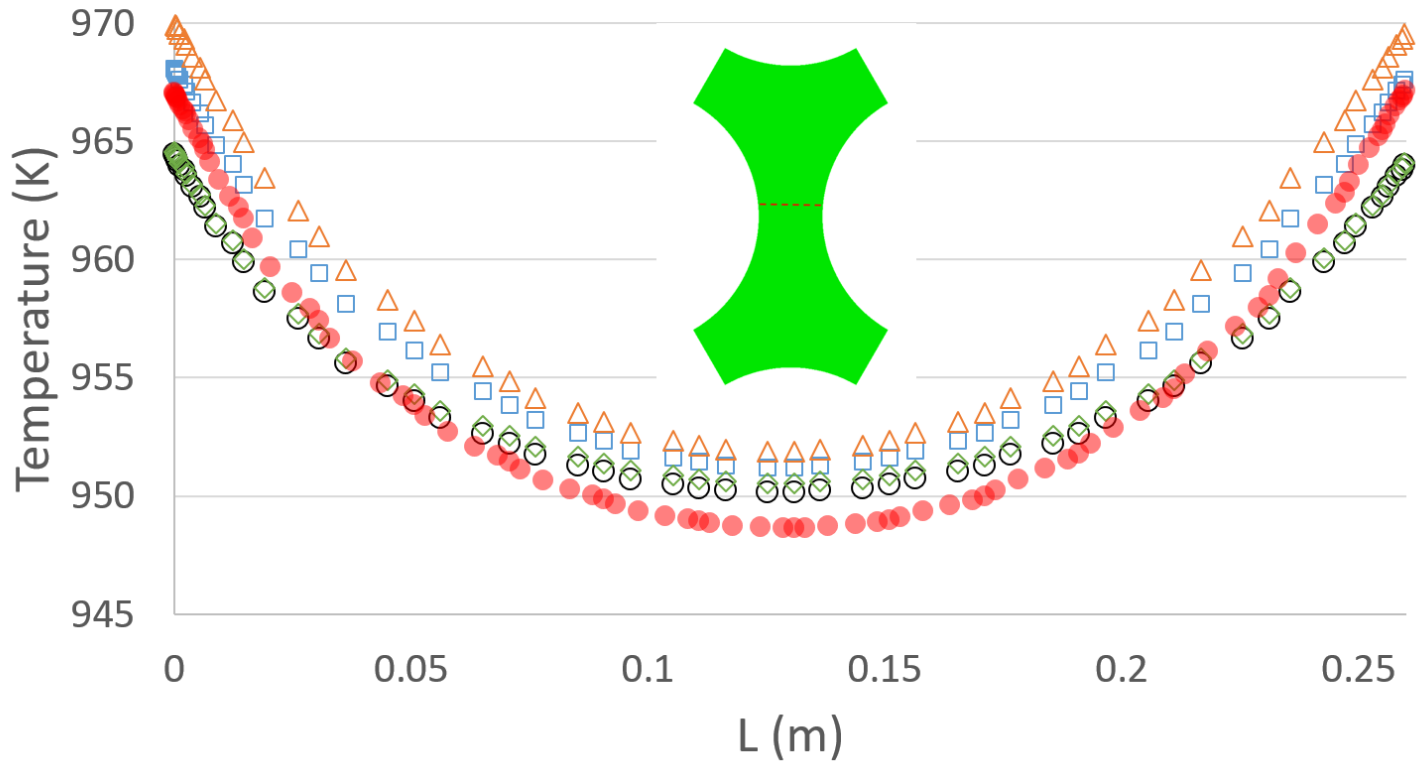

a) Horizontal Line

O Prt $0.9 \square$ Prt $1.5 \triangle$ Prt $2 \diamond$ Prt local $\bullet$ LES

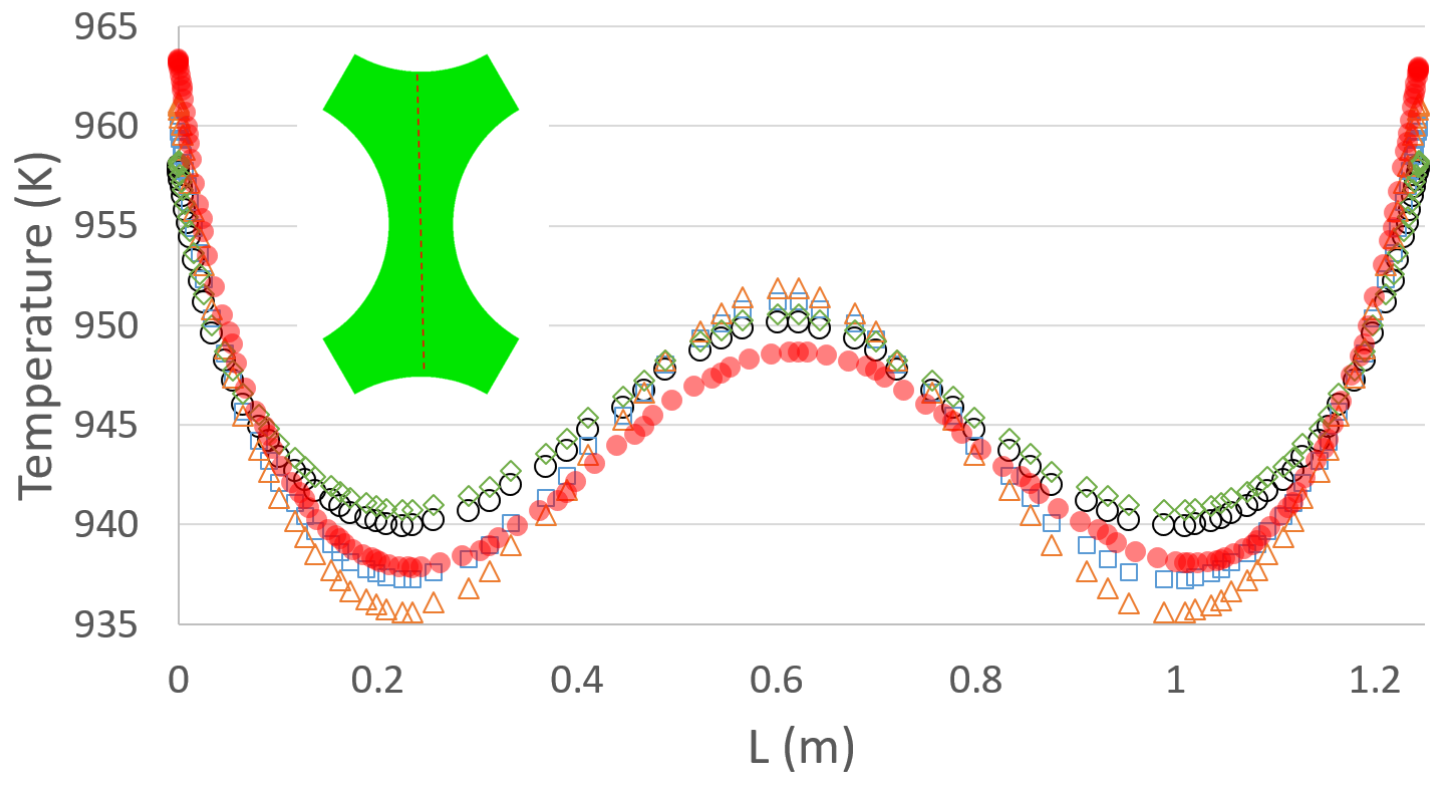

b) Vertical Line

Figure 2.5.6 Temperature distribution between rods with varying turbulent Prandtl number 


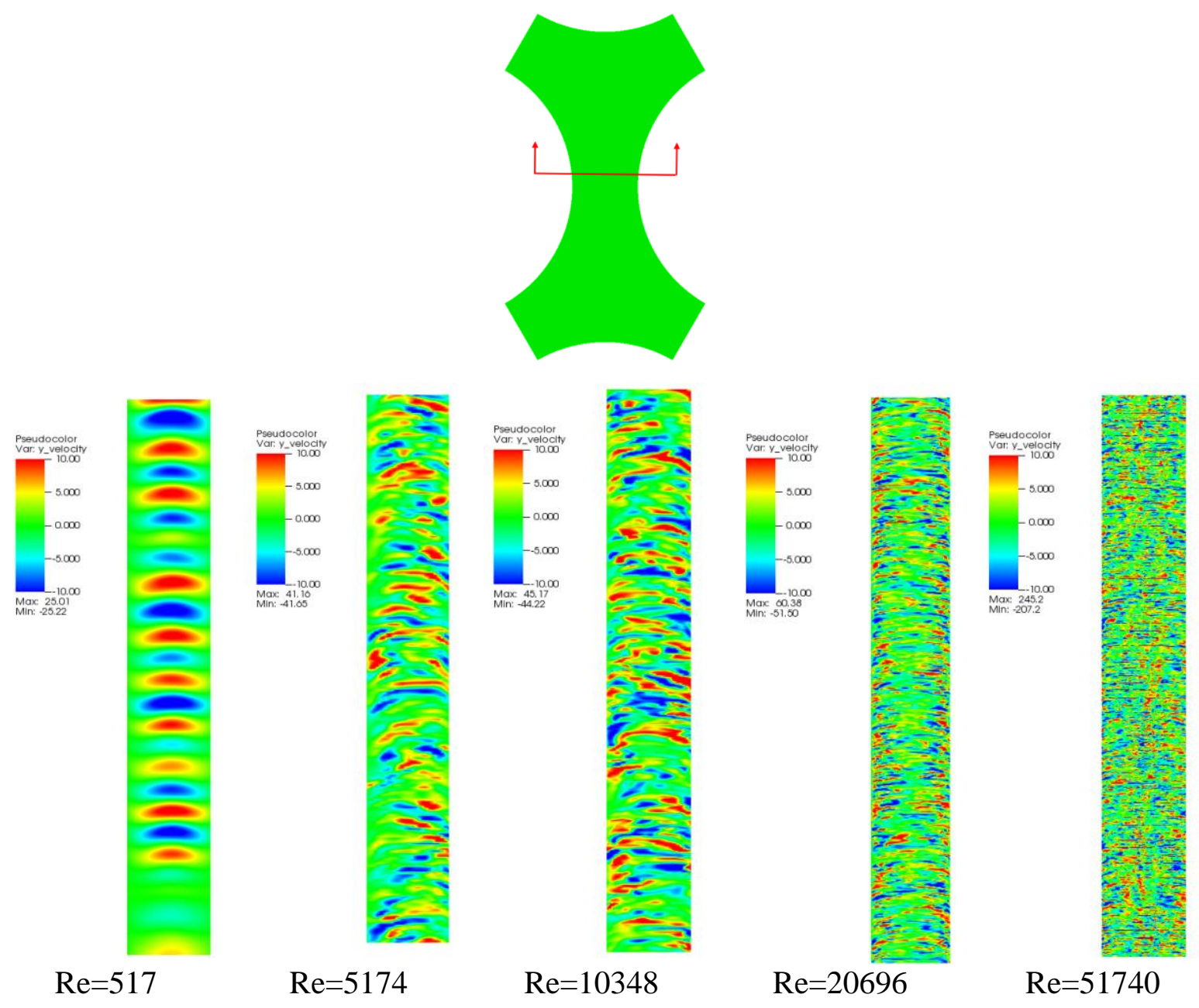

Figure 2.5.7 Flow oscillation in the gap with different Reynolds number

The high fidelity LES simulations are performed to select the best turbulent Prandtl number for this specific LFR design. Based on the analysis mentioned above, URANS model with $\operatorname{Pr}_{t}=1.5$ predicts the most accurate maximum temperature and reasonable global Nusselt Number. Thus, $\operatorname{Pr}_{t}=1.5$ will be used for further HCF calculations.

\subsection{HCF Associated with Uncertainties in Cladding Thickness}

The cladding material is assumed to be double-stabilized austenitic stainless steel (DS4) with a manufacturing tolerance of $\pm 0.05 \mathrm{~mm}$. While the variations in cladding thickness should ideally be modeled stochastically for each pin, this requires an extraordinary amount of mesh preprocessing effort and different number of cases. Therefore, the two bounding cases were modeled ( $-0.05 \mathrm{~mm}$ cladding thickness in all pins and $+0.05 \mathrm{~mm}$ cladding thickness in all pins) in Nek5000. Only the reduction in cladding thickness case was modeled in neutronics as it was 
found to have no impact on the power distribution. The hot cladding dimensions are summarized in Table 2.6.1.

Table 2.6.1. Cladding perturbation parameters

\begin{tabular}{|c|c|c|c|c|}
\hline Case & $\begin{array}{c}\text { Cold Clad } \\
\text { Thickness } \\
(\mathbf{m m})\end{array}$ & $\begin{array}{c}\text { Hot Clad } \\
\text { Thickness } \\
(\mathbf{m m})\end{array}$ & $\begin{array}{c}\text { Hot Inner } \\
\text { Clad Radius } \\
(\mathbf{m m})\end{array}$ & $\begin{array}{c}\text { Hot Outer } \\
\text { Clad Radius } \\
(\mathbf{m m})\end{array}$ \\
\hline Nominal & 0.900 & 0.909 & 4.495 & 5.404 \\
\hline $\begin{array}{c}\text { Perturbed } \\
(-0.05 \mathrm{~mm} \\
\text { thickness })\end{array}$ & 0.850 & 0.859 & 4.495 & 5.354 \\
\hline $\begin{array}{c}\text { Perturbed } \\
(+0.05 \mathrm{~mm} \\
\text { thickness })\end{array}$ & 0.905 & 0.914 & 4.495 & 5.409 \\
\hline
\end{tabular}

\subsubsection{Neutronics Discussion}

The neutronics results determined that the cladding thickness reduction has a negligible impact on both power shape and magnitude. The nominal and perturbed power shapes in a corner pin (pin 122) are compared in Figure 2.6.1 and shown to be identical. The eigenvalue for the perturbed case did increase from 1.17701 (nominal case) to 1.18121 (perturbed case) but the power details were unaffected given that the power was normalized to the same level as the nominal condition case. 


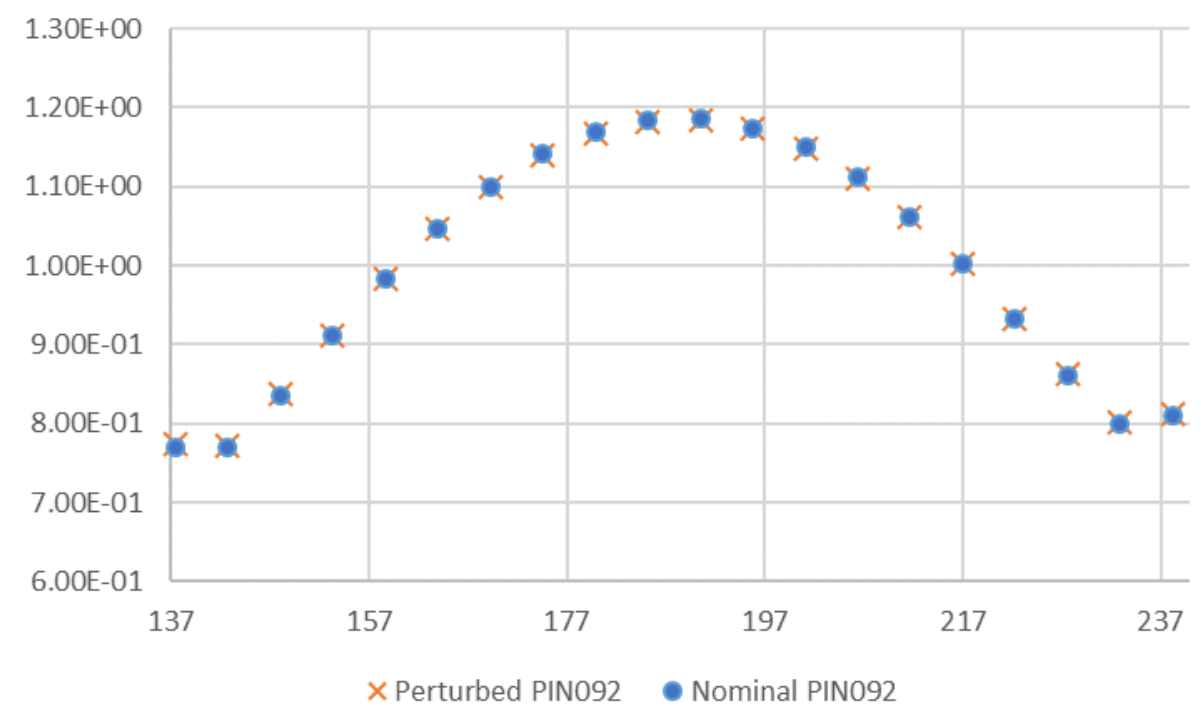

Figure 2.6.1 Nominal and perturbed axial power shapes in pin 122 (outside corner)

A tiny fraction of power was shifted from the outside corner pin fuel and cladding zones to the inner rings, essentially flattening out the power distribution even more than it already was in the nominal condition. The axially integrated (along active fuel zone) fuel and cladding powers are shown in Figure 2.6.2 and 2.6.3. Pin 1 is the center pin, and pins 2-7 are in ring 2, and so forth. Pin 122 is one of the 6 corner pins in the fuel assembly.

The maximum pin power difference occurs in the outer corner pins $(-9 \mathrm{~W}$ reduction in fuel power, $<1 \mathrm{~W}$ reduction in cladding power). This is negligible compared to the total integrated fuel power of the pin itself ( $28.76 \mathrm{~kW}$ per pin). Therefore, the neutronics impact of the cladding perturbation can be completely ignored in terms of power shape and power magnitude in each pin. Since reduction of the cladding thickness did not impact the neutronics solution, the increased cladding thickness case was not performed. 


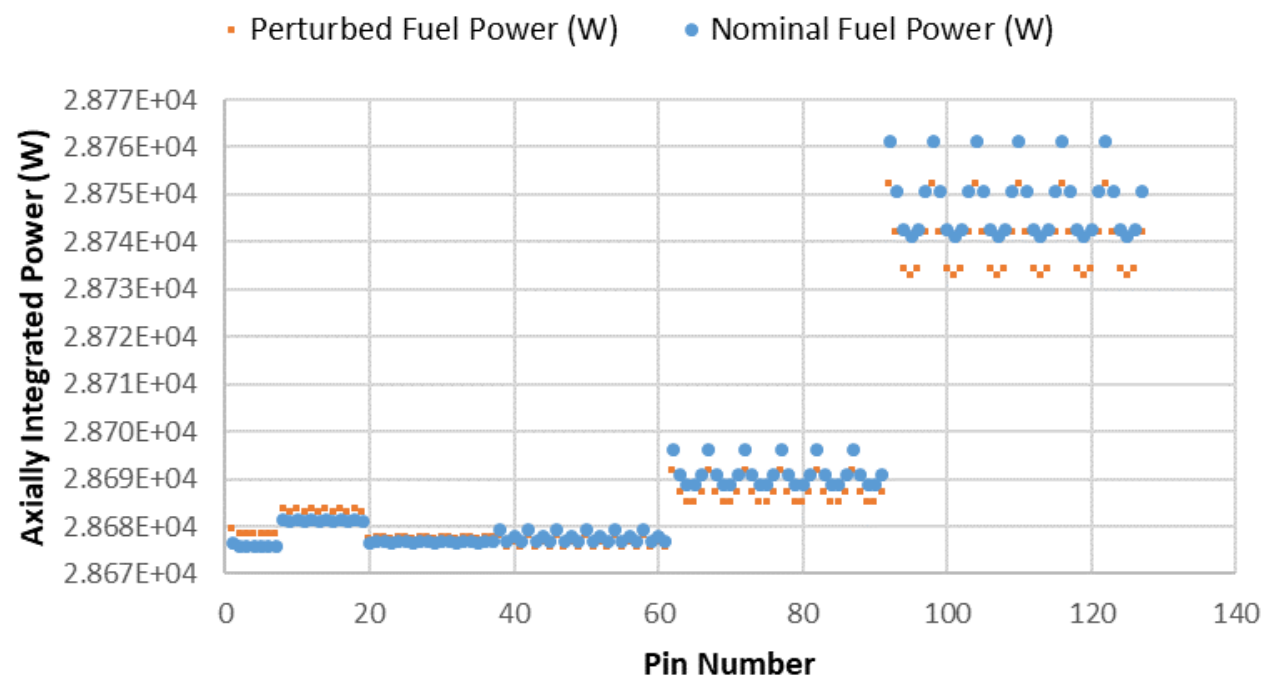

Figure 2.6.2 Nominal and perturbed integrated fuel powers (all pins)

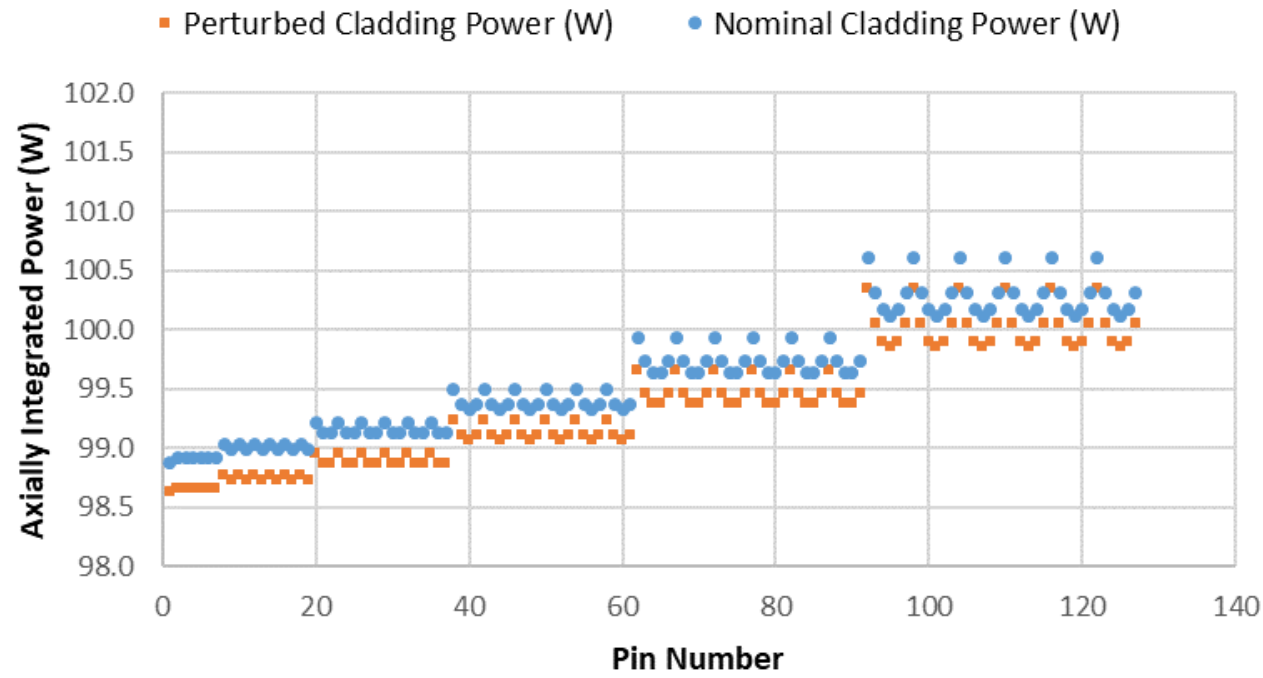

Figure 2.6.3 Nominal and perturbed integrated cladding powers (all pins)

The nominal condition power profile was used in the Nek5000 analysis for the cladding perturbation case. Both the cladding thickness increase and decrease were modeled because the combined effect of the changing coolant subchannel flow area as well as the decreased cladding thickness for heat transfer have undetermined effects. 


\subsubsection{Thermal Hydraulics Discussion}

As a reminder, the uncertainty of the cladding thickness is $\pm 0.05 \mathrm{~mm}$. The uncertainty is applied to all pins. In conventional HCF estimation, the uncertainty of the cladding thickness is taken as an independent factor and is calculated as an analytical solution. In actuality, the change of cladding thickness will have impact on power distribution, flow area, velocity distribution and thermal conduction. All of these effects are coupled together to influence the temperature. Therefore, the temperature change depends on which effects dominate in a specific location. In the high fidelity simulations performed here, these sub-factors are taken into account simultaneously. The resulting HCF calculated will be certainly more accurate than an analytical solution.

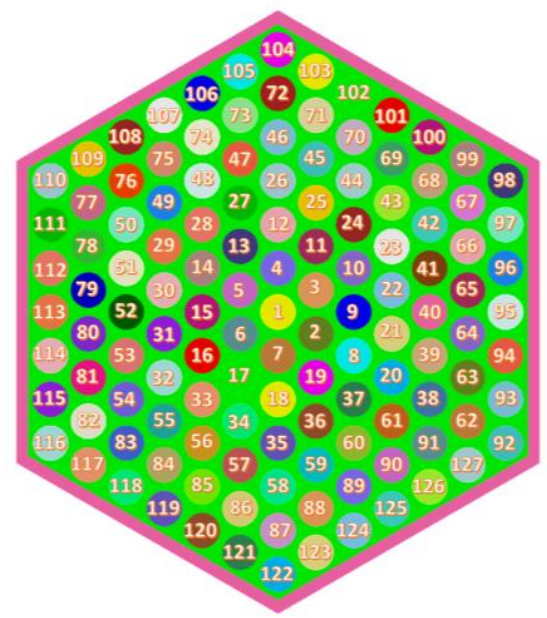

Figure 2.6.4 Numbering scheme of fuel assembly

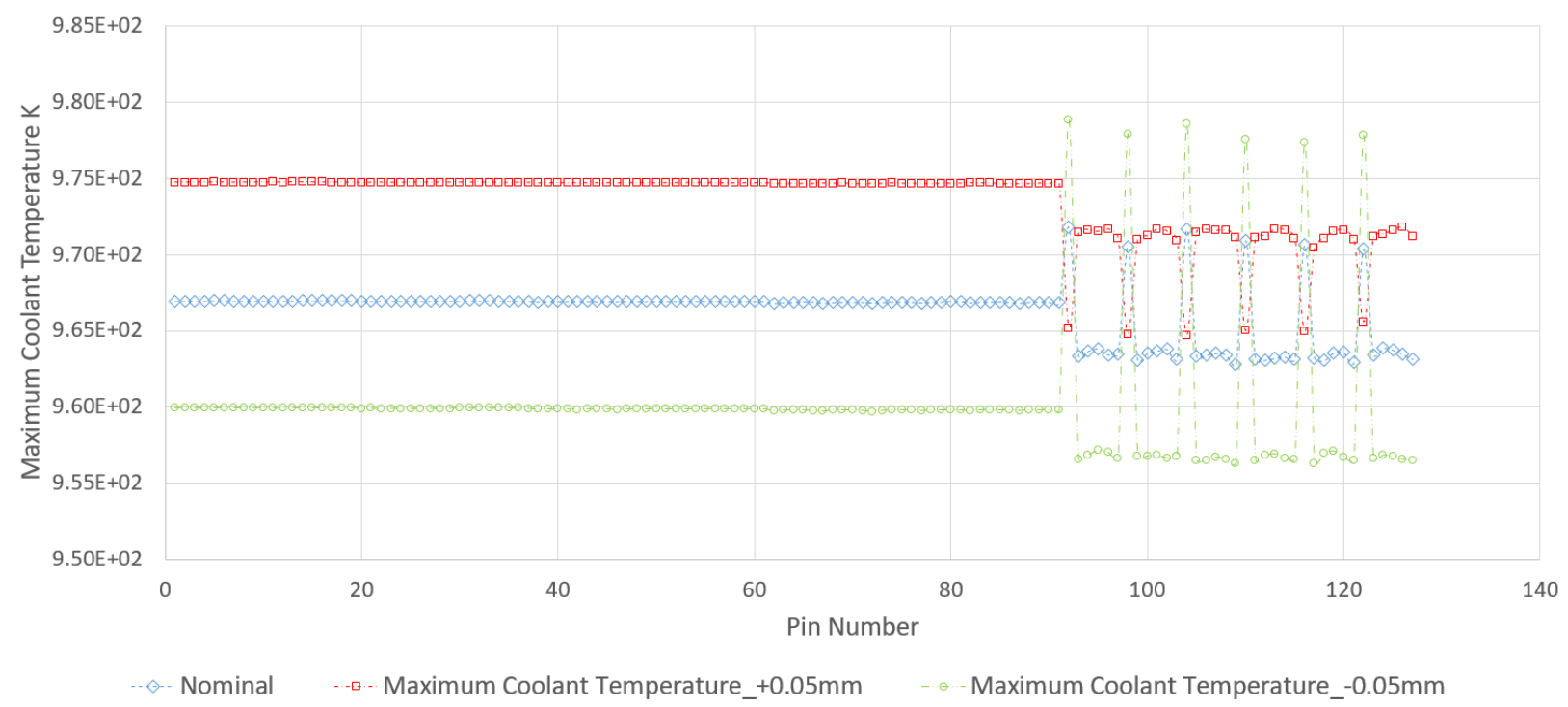

Figure 2.6.5 Maximum coolant temperature responding to the uncertainty of cladding thickness 
Figure 2.6.4 shows the pin numbering in the LFR fuel assembly. Figure 2.6.5 shows the maximum coolant temperature (outer cladding temperature) with maximum cladding thickness uncertainty. In the inner rings (pins 1-91), the maximum coolant temperature responds to the cladding thickness uncertainty in an intuitive way. The larger cladding thickness leads to smaller flow region and thus smaller bulk velocity and higher coolant temperature.

In the outermost ring (pins 92-127), the maximum coolant temperature for most of the pins respond to the cladding thickness uncertainty in the same way whereas the corner pins respond differently. For the corner pin, a larger cladding thickness leads to lower maximum coolant temperature. The heat transfer mechanism in the corner region is dominated by different factors. In the corner, the flow is surrounded by two duct walls, which makes the total shear stress greater than that in other regions. The flow velocity is quite small in that region. Further decreasing the flow here will have a smaller impact on the maximum coolant temperature than it does in other regions. However, increasing the cladding thickness increases the distance for heat conduction through the cladding, which will lead to coolant temperature drop. In other word, the heat transfer mechanism in the corner pin regions is different from that in other regions. The maximum coolant temperature for the corner pin respond to the uncertainty of the cladding thickness counterintuitively. Through the high fidelity coupled simulation, not only the hot channel factor but also the supporting data is obtained. The data can be used to study the physics mechanisms at play. Each hot channel factor is no longer taken as an independent factor and can be studied comprehensively for results that are more reliable.

\subsection{HCF Associated with Uncertainties in Fuel Pellet Fissile Content}

The MOX fuel is specified to be $23 \% \mathrm{PuO}_{\mathrm{x}} /\left(\mathrm{PuO}_{\mathrm{x}}+\mathrm{UO}_{\mathrm{x}}\right)$. The uranium is depleted $(0.25 \mathrm{wt} \%$ $\mathrm{U}-235)$, and the $\mathrm{Pu}$ is enriched (68.769 wt $\% \mathrm{Pu}-239,1.759 \mathrm{wt} \% \mathrm{Pu}-241)$. Due to manufacturing uncertainty, the Pu-239 content has a manufacturing tolerance of $+/-5 \%$ (3-sigma normal distribution) from the target enrichment. This variability in enrichment can causes changes in local pin power and temperature.

\subsubsection{Neutronics Discussion}

A stochastic (random sampling) technique was used to assess the impact of fissile content uncertainty. To obtain meaningful stochastic distributions, thirty independent neutronics inputs were randomly selected as follows:

1. The fissile content in each pin is sampled individually and not affected by neighboring pins.

2. A normal distribution is used to sample each enrichment perturbation, with mean $=0 \%$ and 1sigma $=1.67 \%(3$-sigma $=5 \%)$. In such a distribution, more than $99 \%$ of fuel pins will have enrichments within $5 \%$ of the target manufacturing value.

3. The sampled enrichment perturbations are rounded to the nearest integer in the discrete set $\{ \pm 6$, $\pm 5, \pm 4, \pm 3, \pm 2, \pm 1,0\} \%$. Limiting the enrichment perturbations to integers simplifies the definition of material compositions in neutronics. 
4. The density of the fuel for each pin was assumed constant by modifying Pu-240 content to compensate for changing $\mathrm{Pu}-239$ content.

5. The fuel assembly power was held constant at 3.7 MW for each independent simulation.

The eigenvalues from the thirty simulations (B1-B30) were binned in a histogram chart (Figure 2.7.1) and generally follow a normal distribution. The eigenvalue histogram uses bin widths of $66 \mathrm{pcm}$ (0.00066). The average k-eff is 1.7699 which is within $2 \mathrm{pcm}$ of the nominal condition calculation (1.17701).

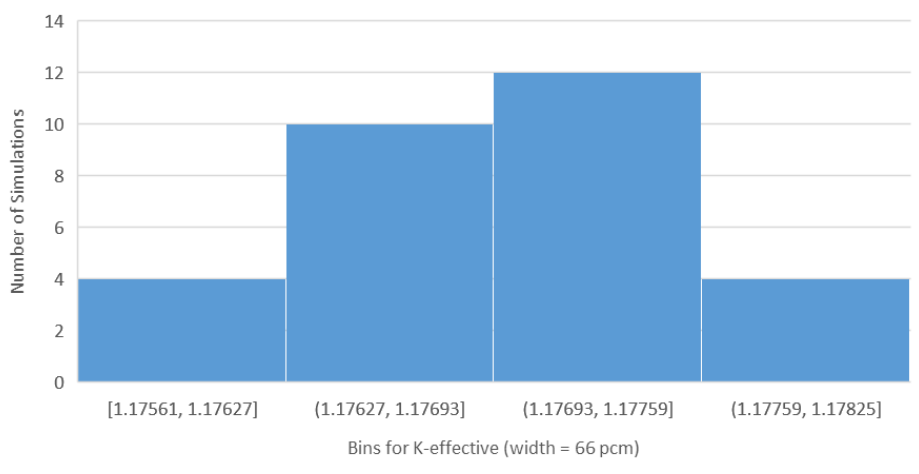

Figure 2.7.1 Distribution of eigenvalues across 30 random samples

Of the thirty independent simulations, Simulation " 2 " yielded the pin with the highest local power, $29.80 \mathrm{~kW}$ in Pin 81 . This value is $1.04 \mathrm{~kW}$ higher (3.6\% higher), and in a different pin location, than the expected maximum pin power of $28.76 \mathrm{~kW}$ in the nominal condition which occurs in corner pins such as Pin 92. A mid-core slice showing the power distribution for the nominal condition and Simulation 2 is shown in Figure 2.7.2. The nominal case assumes all pins are manufactured with the targeted $\mathrm{Pu}-239$ content. Simulation 2 contains pins with randomized fissile content perturbations according to the normal distribution described in this section. The color scale is identical in the two figures. The uniform pin powers in the nominal condition are in stark contrast to the highly variable powers in the stochastic simulation. 

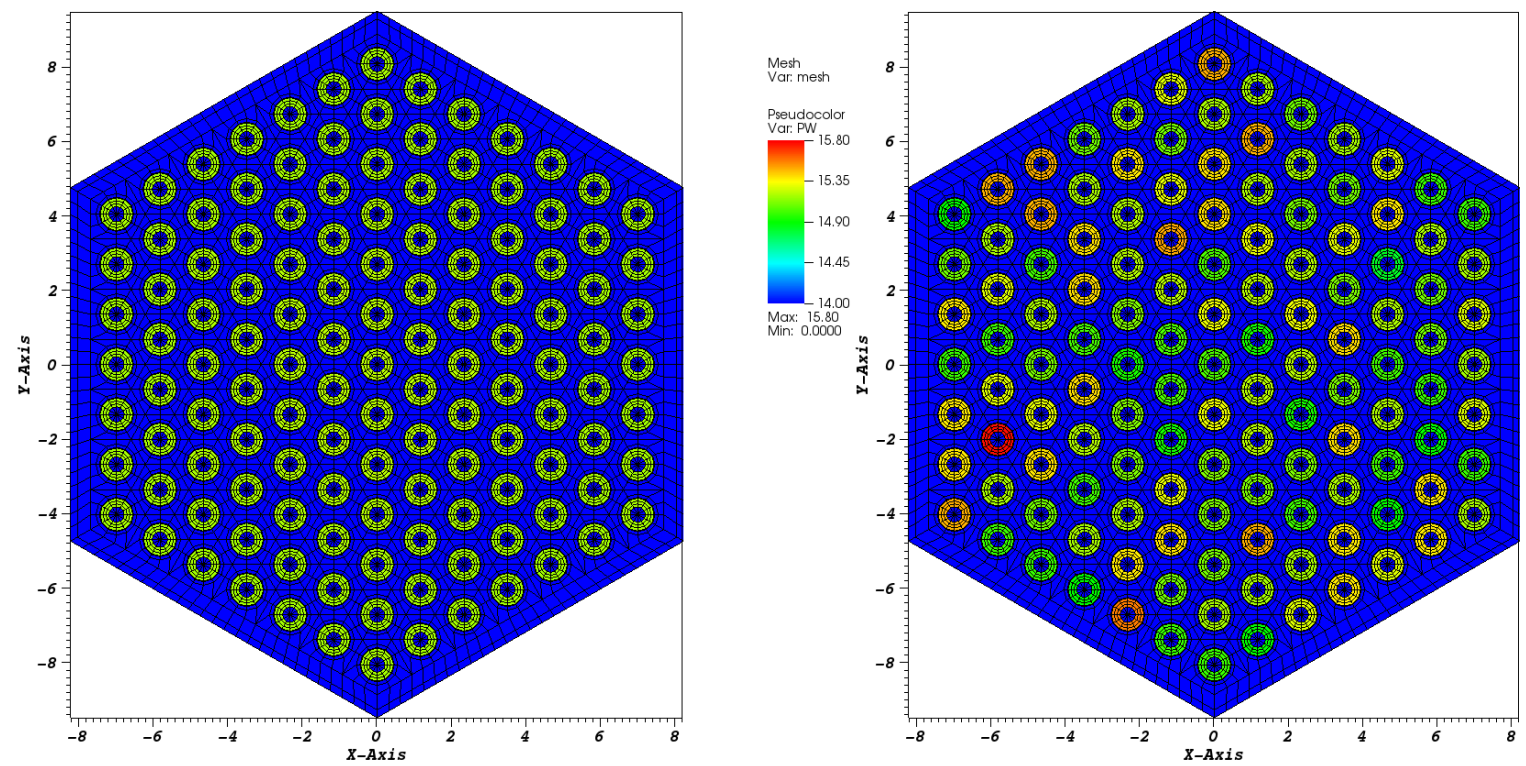

Figure 2.7.2 Power distribution at core mid-plane in nominal condition (left) and randomized fissile content bounding case in Simulation 2 (right).

In Simulation 2, Pin 81 was stochastically assigned an enrichment perturbation of $+6 \%$ which is outside the 3 -sigma range of the distribution ( $99.73 \%$ confidence interval). However, given the 3,810 enrichment perturbation sampled (127 pins/sample*30 samples), about $1 \%$ or 10 samples are expected to lie outside this interval.

The distribution of enrichment perturbations for Simulation 2 is depicted in Figure 2.7.3 as a histogram. Most of the samples are clustered within $[-3,+3]$, which makes sense because the range $[-3.33,+3.33]$ represents the 2 -sigma interval $(95.45 \%$ confidence level). Therefore, the distribution looks qualitatively valid. Figure 2.7.4 depicts the distribution of pin powers in Simulation 2 . The peak pin power in pin 81 is clearly identified. 


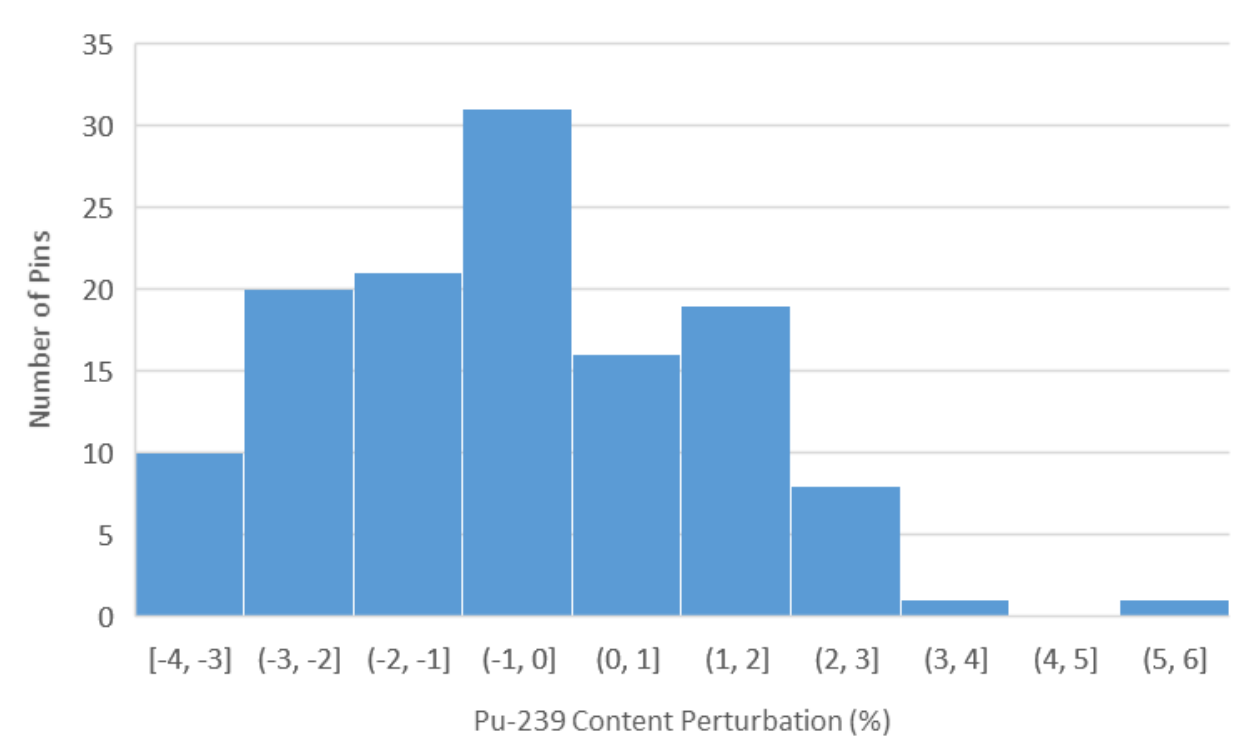

Figure 2.7.3 Distribution of enrichment perturbations for pins in Simulation 2 (simulation with maximum pin power)

\section{Simulation 2}

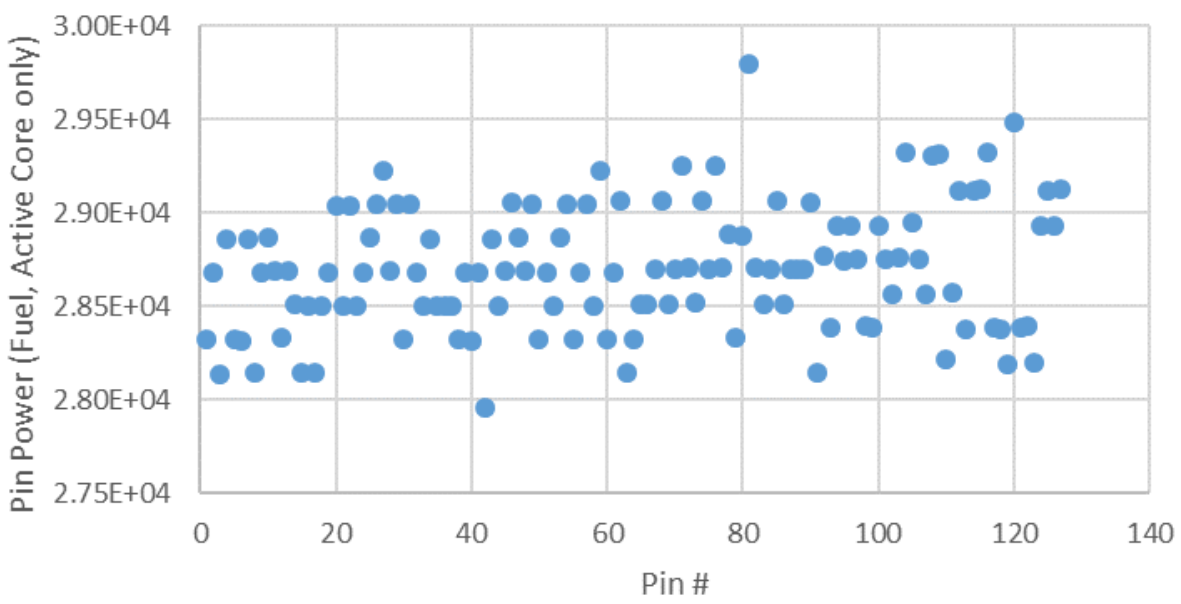

Figure 2.7.4 Distribution of pin powers in Simulation 2 (simulation with maximum pin power)

The normalized axial power distribution for each pin needed for Nek5000 is plotted in Figure 2.7.5. The selected pins have the following enrichments perturbations and locations: Pin 1 (-2\%, ring 1), Pin 42 (-4\%, ring 4), Pin 81 (+6\%, ring 6), Pin 106 (+0\%, ring 7). The figure demonstrates that the axial power shape is not significantly impacted by the local enrichment perturbations or by pin location. Therefore, the same axial power shape can be used for all of the pins in Nek5000, where the scaling factor (pin power) is unique. 


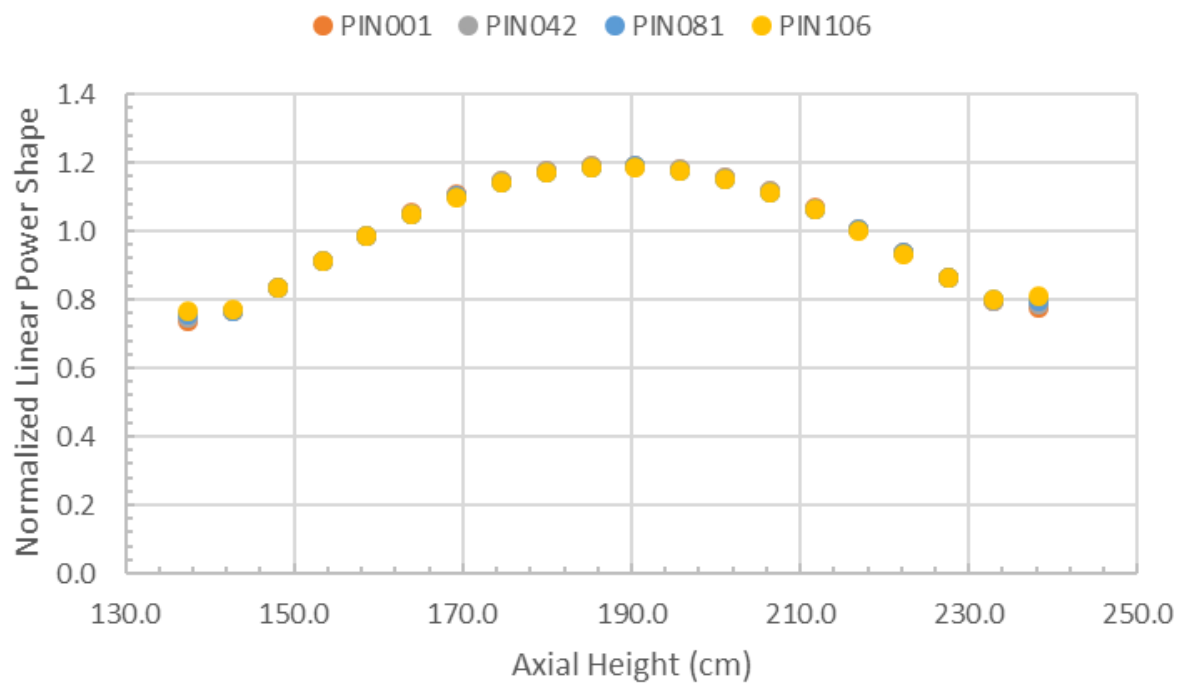

Figure 2.7.5. Normalized axial power distribution for selected pins within Simulation 2

A follow-on calculation (C) was carried out to identify the impact of directly changing the corner pin (Pin 92)'s Pu-239 content by $+5 \%$. This could be considered a "direct" rather than "statistical" calculation of the same hot channel factor. In the direct calculation, the maximum power was calculated to be $29.68 \mathrm{~kW}$ in Pin 92 . This value is lower than the pin power observed from the stochastic simulations, therefore it is less conservative.

Table 2.7.1. Summary of fissile maldistribution impact on peak pin power

\begin{tabular}{|l|c|c|}
\hline Case & Location & Peak Pin Power (kW) \\
\hline (A) Nominal & $\begin{array}{c}\text { Pin 92 } \\
\text { (ring 7) }\end{array}$ & 28.76 \\
\hline $\begin{array}{l}\text { (B1-B30) Stochastic; all pins perturbed } \\
\text { according to normal distribution with 3- } \\
\text { sigma = 5\% }\end{array}$ & $\begin{array}{l}\text { Pin 81 } \\
\text { (ring 6) }\end{array}$ & 29.80 \\
\hline (C) Direct; Pin 92 perturbed by 5\% & $\begin{array}{l}\text { Pin 92 } \\
\text { (ring 7) }\end{array}$ & 29.68 \\
\hline
\end{tabular}




\subsubsection{Thermal Hydraulics Discussion}

In the past, due to the lack of computational resource, only the neutronics simulation with the maximum peak pin power was conservatively chosen to transfer power to Nek5000. Development of Nek5000 during FY19 enabled improved computational efficiency, and consequently, all thirty cases were simulated in Nek5000. Due to the relatively small number of samples (30), the simulation with the maximum peak temperature was chosen to estimate hot channel factor. A normal distribution would be more likely to occur with more random sampling and the simulation with a peak pin power lying in the $95 \%$ confidence interval (2-sigma) could be selected as shown in Figure 2.7.6.

The observed distribution of peak power and maximum cladding temperature is shown in Figure 2.7.7. A more standard normal distribution is expected if more samples are used. The maximum coolant temperature, cladding temperature, fuel temperature and peak power for all 30 cases are presented in Figure 2.7.8 - 2.7.10. The benefit of performing 30 Nek5000 simulations is well explained in these figures. The maximum peak power occurs in neutronics Simulation 2, corresponding to T/H Case 2. However, the maximum temperature in coolant, cladding and fuel does not all occur in Case 2. The maximum coolant temperature occurs in Case 13 rather than Case 2. This is because the maximum temperature depends on not only the magnitude of the peak power but also the position of the peak power. Although the results show that the maximum cladding temperature and fuel temperature still shows in Case 2 (red frame in Figure 2.7.9), they also demonstrate that higher peak power does not automatically lead to higher maximum temperature (green frame in Figure 2.7.9 showing a high cladding temperature despite the lower peak pin powers compared to the rest of the simulations). Table 3 that lists the rank of peak power and maximum coolant, cladding and fuel temperature rise for all 30 cases can further demonstrate that in detail. Similar results are observed in Figure 2.7.8 and Figure 2.7.10 as well.

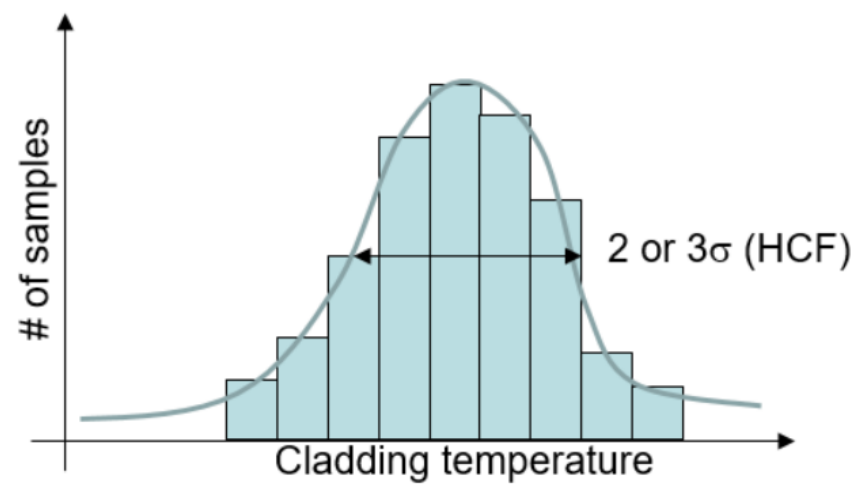

Figure 2.7.6 Hypothetical peak pin power distribution based on random sampling with a very large number of samples. 
12

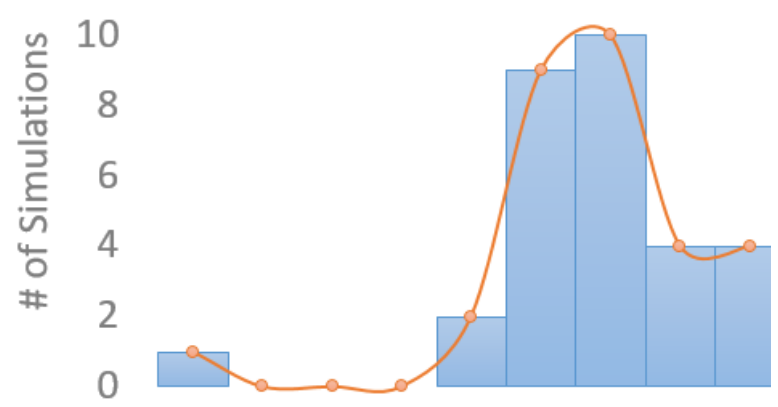

Peak Power
12

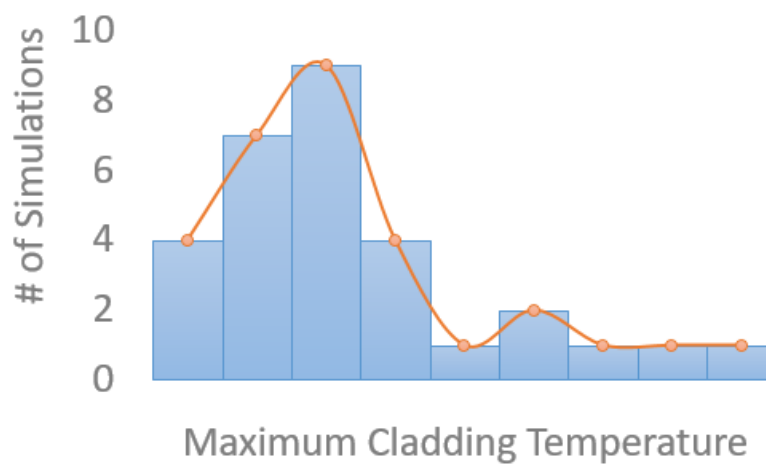

Figure 2.7.7 Distribution of peak power and maximum cladding temperature

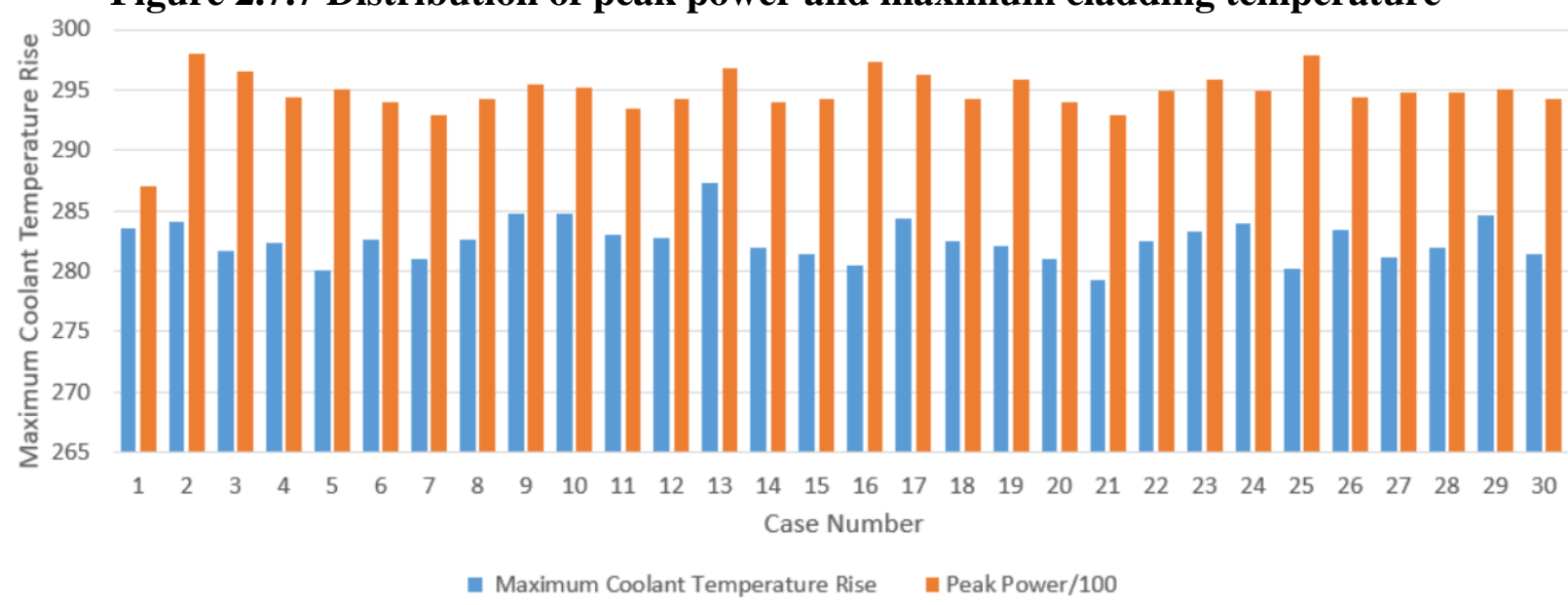

Figure 2.7.8 Maximum coolant temperature and peak power for all 30 cases

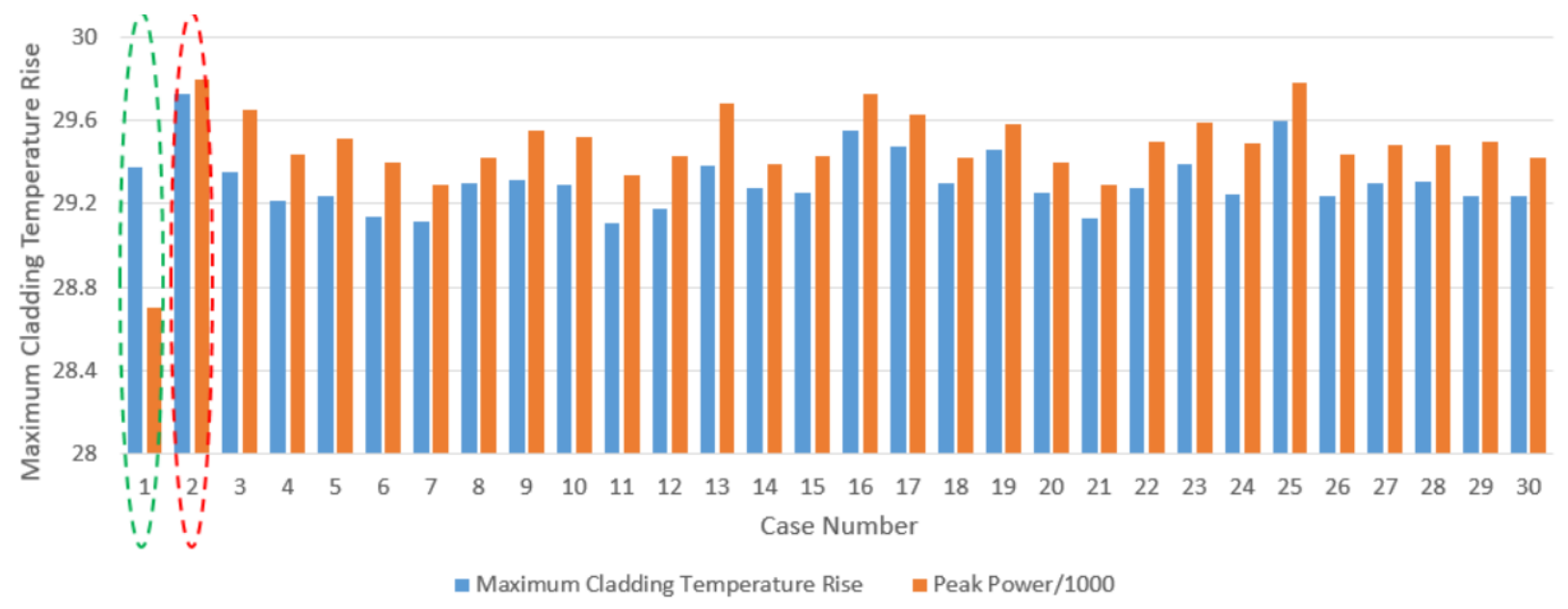

Figure 2.7.9 Maximum cladding temperature and peak power for all 30 cases 


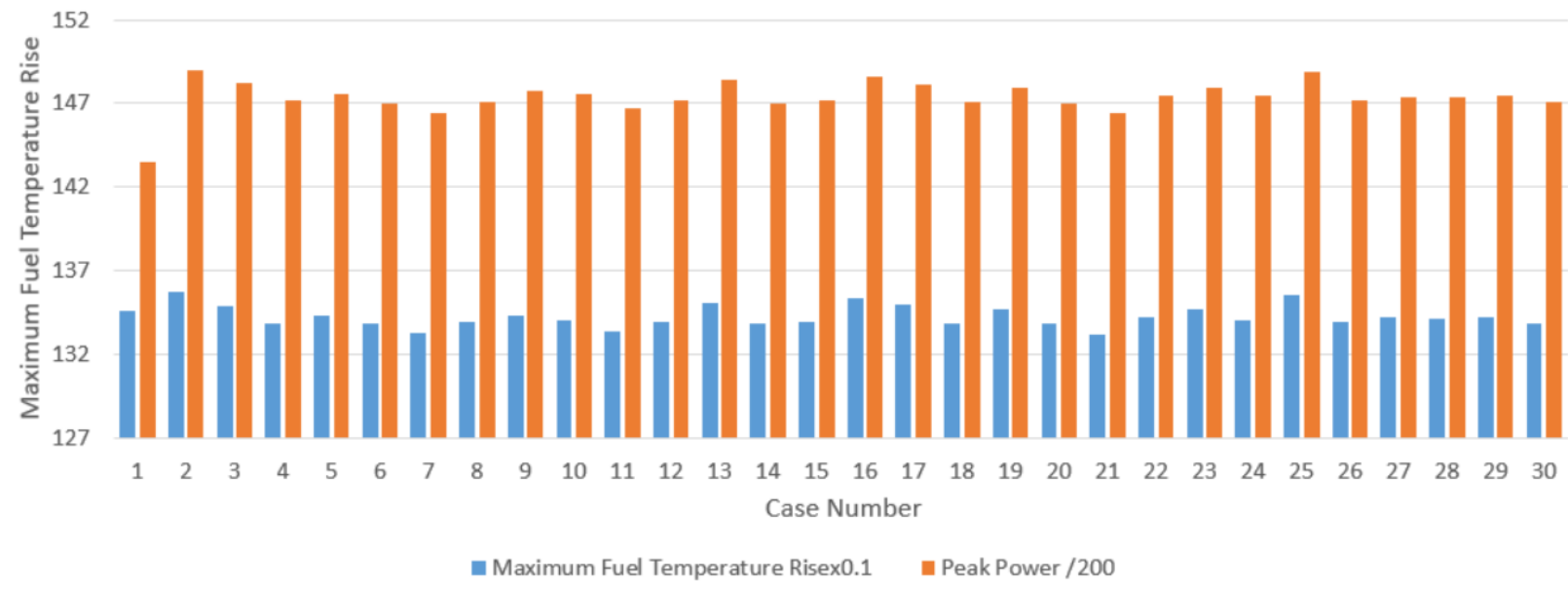

Figure 2.7.10 Maximum fuel temperature rise and peak power for all 30 cases

Table 2.7.2 Rank of peak power, coolant, cladding and fuel temperature rise for all 30 cases

\begin{tabular}{|c|c|c|c|c|c|}
\hline $\begin{array}{c}\text { case } \\
\text { number }\end{array}$ & $\begin{array}{c}\text { Peak Power } \\
\text { x1000 }\end{array}$ & Peak Power Rank & $\begin{array}{c}\text { Maximum Coolant } \\
\Delta \mathrm{T} \text { Rank }\end{array}$ & $\begin{array}{c}\text { Maximum Cladding } \\
\Delta \mathrm{T} \text { Rank }\end{array}$ & $\begin{array}{c}\text { Maximum Fuel } \\
\Delta T \text { Rank }\end{array}$ \\
\hline 1 & 29.80 & 30 & 8 & 8 & 9 \\
\hline 2 & 29.65 & 1 & 6 & 1 & 1 \\
\hline 3 & 29.44 & 5 & 21 & 9 & 6 \\
\hline 4 & 29.51 & 17 & 17 & 25 & 24 \\
\hline 5 & 29.40 & 11 & 29 & 21 & 11 \\
\hline 6 & 29.29 & 25 & 13 & 27 & 27 \\
\hline 7 & 29.42 & 28 & 25 & 29 & 29 \\
\hline 8 & 29.55 & 21 & 14 & 13 & 18 \\
\hline 9 & 29.52 & 9 & 3 & 10 & 10 \\
\hline 10 & 29.34 & 10 & 2 & 15 & 16 \\
\hline 11 & 29.43 & 27 & 11 & 30 & 28 \\
\hline 12 & 29.68 & 19 & 12 & 26 & 21 \\
\hline 13 & 29.39 & 4 & 1 & 7 & 4 \\
\hline 14 & 29.43 & 26 & 19 & 16 & 26 \\
\hline 15 & 29.73 & 20 & 22 & 18 & 19 \\
\hline 16 & 29.63 & 3 & 27 & 3 & 3 \\
\hline 17 & 29.42 & 6 & 5 & 4 & 5 \\
\hline 18 & 29.58 & 22 & 16 & 14 & 22 \\
\hline 19 & 28.70 & 8 & 18 & 5 & 8 \\
\hline 20 & 29.40 & 24 & 26 & 19 & 23 \\
\hline 21 & 29.29 & 29 & 30 & 28 & 30 \\
\hline 22 & 29.50 & 13 & 15 & 17 & 12 \\
\hline 23 & 29.59 & 7 & 10 & 6 & 7 \\
\hline \multicolumn{2}{|c|}{ ANL/NSE-20/25 } & & 48 & & \\
\hline
\end{tabular}




\begin{tabular}{lccccc}
24 & 29.49 & 14 & 7 & 20 & 17 \\
25 & 29.78 & 2 & 28 & 2 & 2 \\
26 & 29.43 & 18 & 9 & 22 & 20 \\
27 & 29.48 & 16 & 24 & 12 & 13 \\
28 & 29.48 & 15 & 20 & 11 & 15 \\
29 & 29.50 & 12 & 4 & 23 & 14 \\
30 & 29.42 & 23 & 23 & 24 & 25 \\
\hline
\end{tabular}

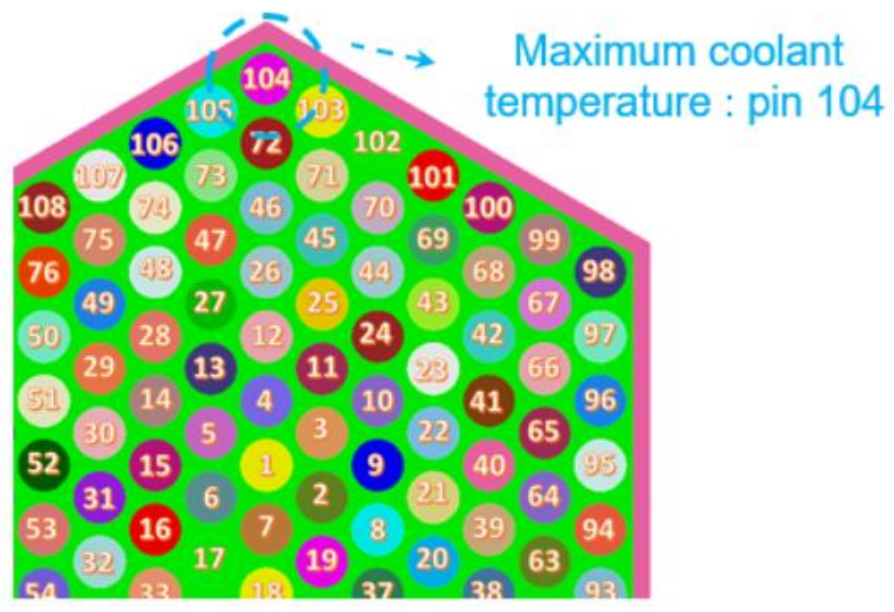

Figure 2.7.11 Location of maximum coolant temperature for Case 2

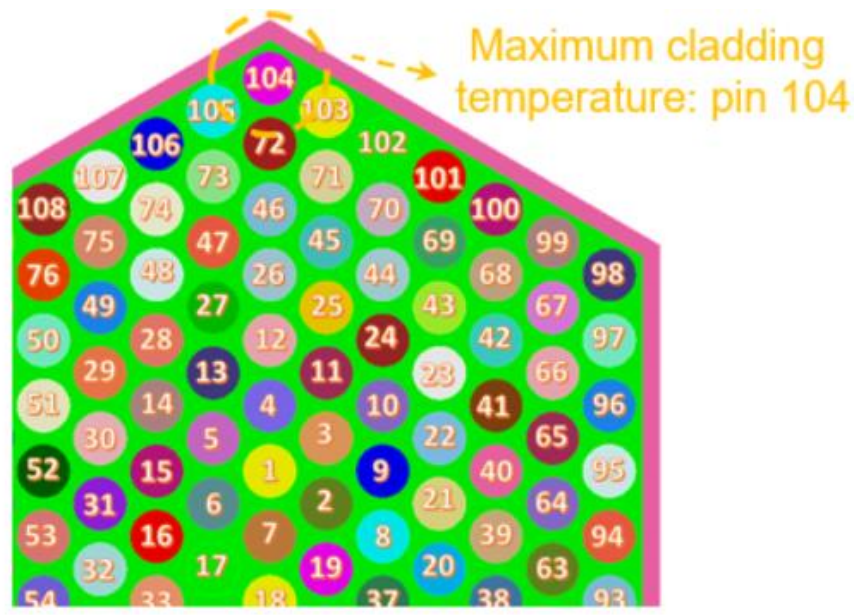

Figure 2.7.12 Location of maximum cladding temperature for Case 2 


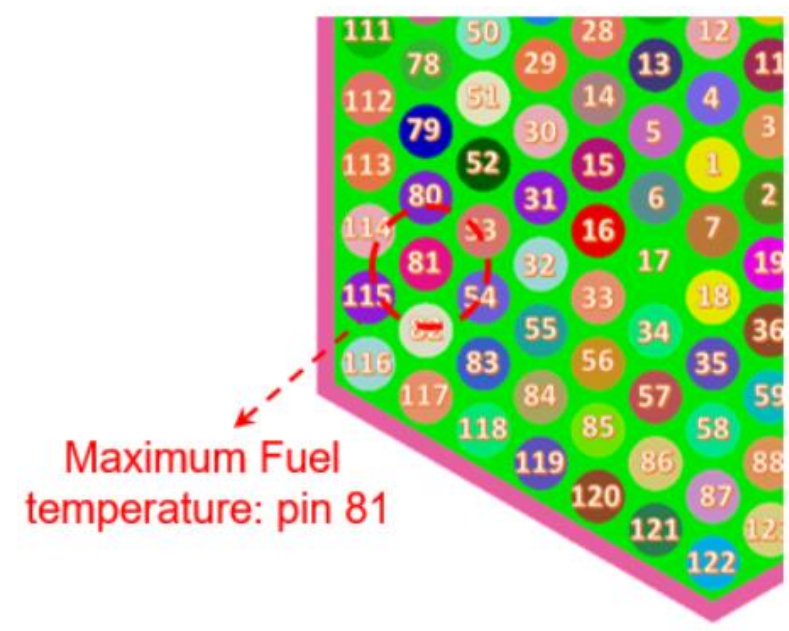

Figure 2.7.13 Location of maximum fuel temperature for Case 2

A detailed simulation for $\mathrm{HCF}$ estimation produces a large amount of data for critical parameters of the target reactor. The simulation data can be used for mechanism studies to help the designer better understand the impact of the uncertainty. Moreover, in the process of the HCF calculation, some improvement or optimization may be found to be necessary. For instance, the locations of maximum coolant, cladding and fuel temperature for Case 2 (which has the maximum peak power among all 30 cases) is shown in Figures 2.7.11 - 2.7.13. The maximum coolant temperature does not show up in the center of the assembly as per the usual case. It shows up in the corner of the assembly. It is not easy to predict that before conducting the simulation. However, in this LFR design, the gap between pins and duct is relatively small, which results in small bulk velocity in the corner of the assembly. The heat transfer is thus reduced, which leads to higher coolant temperature. In the fuel pellet, the peak power and circumferential conduction take its role. The maximum fuel temperature appears in Pin 81 which has peak power. The precise location of the hot spot for cladding (Pin 104) and fuel (Pin 81) are obtained from the simulation. This information can be of interest to industry to optimize their design.

\subsection{HCF Associated with Uncertainties in Coolant Specific Heat}

\subsubsection{Neutronics Discussion}

No neutronics simulations were necessary to examine the impact of uncertainties in coolant specific heat since this is purely thermal hydraulic parameters. Therefore, the nominal condition power solution was used for the thermal hydraulic analysis of this hot channel factor.

\subsubsection{Thermal Hydraulics Discussion}

The HCF for coolant properties due to the uncertainties from material impurity or empirical correlations is important in thermal hydraulic aspect. The lead coolant is assumed to have a specific heat value of $144 \mathrm{~J} / \mathrm{kg} \cdot \mathrm{k}$ with physical uncertainty of $-5 \%$. Lower specific heat value can 
cause less heat carried by the coolant, which leads to higher maximum coolant temperature. To assess the HCF due to uncertainties in material properties, the coolant specific heat was modified directly in the code performing the calculations; by doing so, the effects of uncertainties are properly taken into account.

\subsection{HCF Associated with Uncertainties in Coolant Density}

The mass density of lead $(\mathrm{g} / \mathrm{cc})$ as a function of temperature is given by the correlation [Kirshenbaum 1961], where $\mathrm{T}$ is in degrees Kelvin.

$$
\rho(T)=10.678-13.174 \times 10^{-4}(T-600.6)
$$

The nominal density used in this study is $10.402 \mathrm{~g} / \mathrm{cc}$, which corresponds to a temperature of $810 \mathrm{~K}$ using the previous expression. The coolant temperature in the active fuel zone of the Westinghouse LFR is stated to be $808 \mathrm{~K}$, which is consistent with the temperature correlated to the nominal lead density value. The suggested uncertainty for lead density is $+/-0.8 \%$. Reduction of the coolant density lowers the heat transfer capacity from fuel rod to coolant, and therefore leads to higher temperatures. A case in which lead density was reduced by $0.8 \%(10.319 \mathrm{~g} / \mathrm{cc})$ was analyzed.

\subsubsection{Neutronics Discussion}

The lead coolant density was reduced uniformly to $10.319 \mathrm{~g} / \mathrm{cc}$. Heterogeneously modeled regions such as the active core directly used the reduced lead density, and homogeneous compositions were re-calculated using the reduced lead density and appropriate volume fractions. The density input parameters and eigenvalues are summarized in Table 2.9.1.

Table 2.9.1 Comparison of neutronics for nominal and reduced lead density cases

\begin{tabular}{|l|c|c|}
\hline & $\begin{array}{c}\text { Nominal } \\
\text { Condition }\end{array}$ & $\begin{array}{c}\text { Reduced } \\
\text { Density }\end{array}$ \\
\hline Density (g/cc) & 10.4020 & 10.319 \\
\hline Pb-208 wt\% & $52.4 \%$ & $52.4 \%$ \\
\hline Pb-208 density (at/b-cm) & $1.5840 \mathrm{E}-02$ & $1.5714 \mathrm{E}-02$ \\
\hline k-effective & 1.17701 & 1.17719 \\
\hline Delta-k (pcm) from Nominal & - & 18 \\
\hline
\end{tabular}

In the reduced density case, the ratio of lead isotopes within the lead stay the same relative to each other but the total amount of lead in the system is reduced. The reduced density of lead 
increases the mean free path of neutrons in the system, which consequently increases leakage, but also reduces the moderation and absorption reaction rates of neutrons in lead. Since only a single fuel assembly with reflective boundary conditions is studied for this case, the radial leakage that would occur in the full core cannot be estimated with this model. The combination of increased axial leakage and reduced interactions with lead nuclei serve to increase k-effective slightly $(+19 \mathrm{pcm})$ for a reflected fuel assembly. In a full core calculation, radial leakage would yield a negative change in eigenvalue, so no conclusions can be drawn regarding the k-eff of the full core without knowing the magnitudes of the perturbed leakage and reaction rates. We can only conclude that changing the coolant density slightly does not appreciably change the eigenvalue for an infinite lattice of assemblies.

In terms of power distribution, reducing the lead density does not appreciably change the axial or radial power distribution. As shown in Figure 2.9.1, the integrated pin powers vary by less than $0.003 \%$ from the nominal condition which is insignificant. Figure 2.9.2 shows that the reduced lead density does impact the axial power distribution by a very small amount, shifting power slightly to the top and bottom of the active core. The figure displays the data for the center pin, and the same behavior is present in the outer ring of pins. However, the change from nominal is exceedingly small $(<0.1 \%$ at any given axial plane) and would not impact the location and temperature of the hottest pin. The nominal power distribution can therefore be used for the thermal hydraulics calculation.

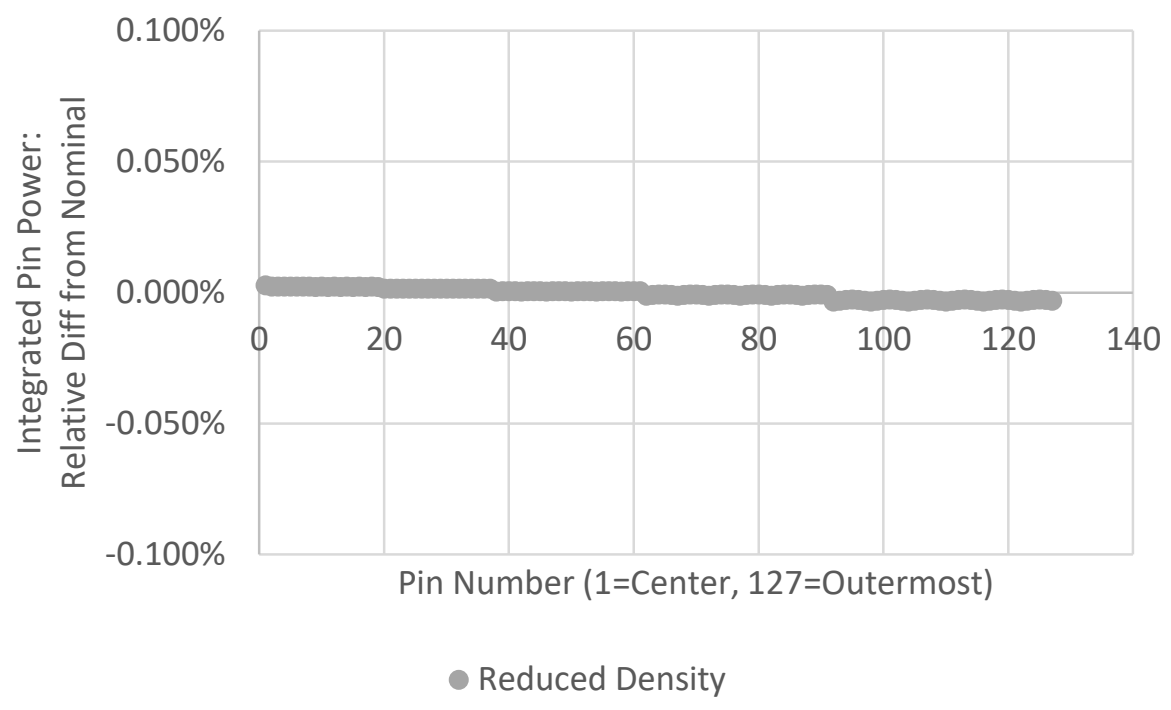

Figure 2.9.1 Relative difference in integrated pin power (reduced lead density case vs. nominal) 


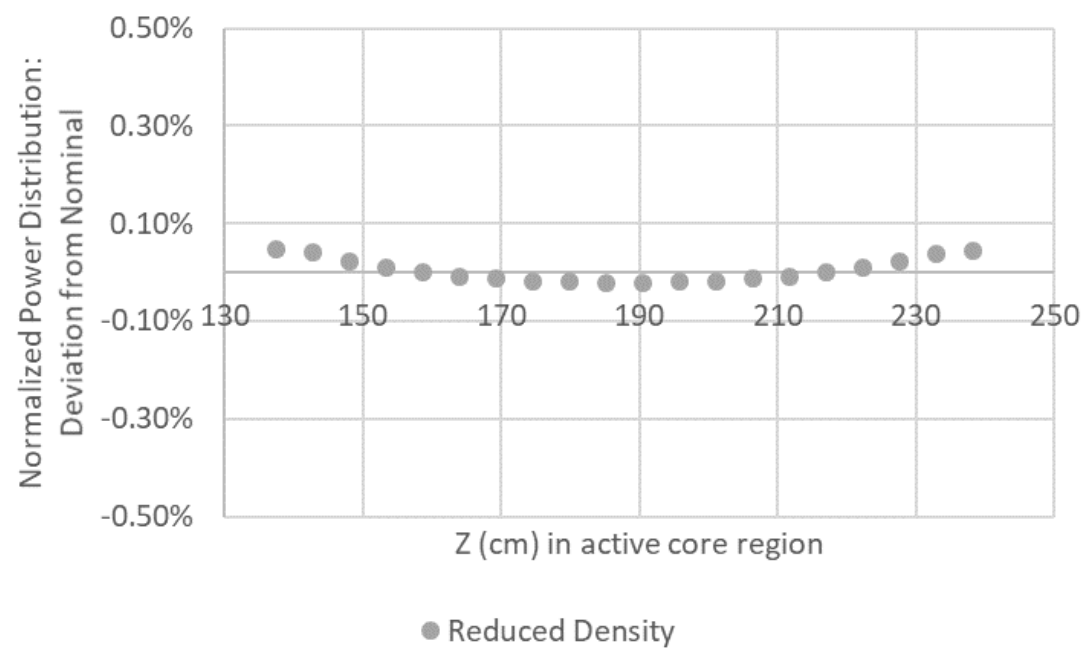

Figure 2.9.2 Relative difference in normalized axial power, center pin (reduced lead density case vs. nominal)

\subsubsection{Thermal Hydraulics Discussion}

The neutronics results shows that the uncertainties in coolant density has little impact on the power solution. Therefore, the nominal condition power solution is used for the thermal hydraulic analysis of this hot channel factor. The HCF for coolant density is due to the uncertainties from material impurity or empirical correlations. The lead coolant is assumed to have a coolant density value of $10401.75 \mathrm{~kg} / \mathrm{m}^{3}$ with physical uncertainty of $0.8 \%$ [Sobolev 2007]. The uncertainty of coolant density can lead to less heat carried by the coolant, which leads to higher maximum coolant temperature. To assess the HCF due to uncertainties in material properties, the coolant density was modified directly in the code performing the calculations; by doing so, the effects of uncertainties are properly taken into account. The resulting hot channel factors are discussed in Section 2.14.

\subsection{HCF Associated with Uncertainties in Coolant Isotopics}

The isotopic composition of natural lead varies significantly due to regional differences in lead mines around the world. The isotopic concentrations in lead coolant will therefore vary depending on the source of lead. Varying lead isotopic composition may impact reactor parameters that are not the focus of hot channel factor analysis, for example the required fuel enrichment. In any case, a confirmatory analysis can be performed to confirm that uniformly changing the lead isotopic ratios does not change the hot spots or temperatures in the assembly, and in the process, the eigenvalue of the reactor can be examined as an additional parameter. 
[Watts 2013] lists several lead ore mines around the globe and the associated concentrations of each lead isotope by percent. The $\mathrm{Pb}-208$ content from the various mines varies from $37.64 \%$ (low quality) to $58.599 \%$ (high quality). There is an opportunity to select higher quality lead, i.e. lead with high $\mathrm{Pb}-208$ content, by selecting specific origin mines (e.g. Canon Diablo, Arizona or Henbury, Australia). $\mathrm{Pb}-208$ has very low cross sections ( $<0.3 \mathrm{mbarn})$ for neutron radiation capture below $50 \mathrm{keV}$ neutron energy, and high threshold $(2.6 \mathrm{MeV})$ for inelastic interaction of neutrons with Pb-208 nuclei [Khorasanov 2013] so is more desirable from a neutron economy perspective.

The impact of variable lead composition was assessed by selecting high quality (Canon Diablo, Arizona) and low quality (Ace Mine, Saskatchewan) leads which provide bounding cases based on $\mathrm{Pb} 208$ content being as large or small as possible. The density of high quality and low quality lead was held constant at $10.402 \mathrm{~g} / \mathrm{cc}$ which is the same as the nominal condition (average quality lead). The atomic densities of each lead isotope were re-calculated to preserve the nominal density and the desired composition.

The effect of fluctuating $\mathrm{Pb}-208$ on pin power distribution, temperature and the core $\mathrm{k}$ effective was assessed. The nominal condition calculation uses "average" quality lead, whose isotopic composition was provided by Westinghouse: $\mathrm{Pb}-204$ (1.4\%), Pb-206 (24.1\%), Pb-207 (22.1\%) and $\mathrm{Pb}-208$ (52.4\%). Table 2.10.1 summarizes the properties of the nominal lead, high quality lead, and low quality lead.

Table 2.10.1: Isotopic concentrations of various lead mines by percent [Watts 2013]

\begin{tabular}{|c|c|c|c|c|c|c|}
\hline & \multicolumn{2}{|c|}{ Nominal Condition } & \multicolumn{2}{|c|}{ High Quality } & \multicolumn{2}{|c|}{ Low Quality } \\
\hline Lead Source & \multicolumn{2}{|c|}{ Average value } & \multicolumn{2}{|c|}{$\begin{array}{c}\text { Canon Diablo, AZ, } \\
\text { USA }\end{array}$} & \multicolumn{2}{|c|}{ Ace Mine, SK, CAN } \\
\hline $\begin{array}{l}\text { (g/cc) } \\
\text { (g/ty }\end{array}$ & & 0.402 & & .402 & & .402 \\
\hline Isotopes & wt\% & $\begin{array}{c}\text { Density } \\
\text { (at/b-cm) }\end{array}$ & wt\% & $\begin{array}{c}\text { Density } \\
\text { (at/b-cm) }\end{array}$ & wt\% & $\begin{array}{c}\text { Density } \\
\text { (at/b-cm) }\end{array}$ \\
\hline Pb204 & 1.4 & 4.2322E-04 & 1.990 & $6.0129 \mathrm{E}-04$ & 1.044 & 3.1606E-04 \\
\hline Pb206 & 24.1 & 7.2854E-03 & 18.830 & $5.6896 \mathrm{E}-03$ & 41.870 & $1.2676 \mathrm{E}-02$ \\
\hline Pb207 & 22.1 & $6.6808 \mathrm{E}-03$ & 20.581 & $6.2186 \mathrm{E}-03$ & 19.450 & $5.8884 \mathrm{E}-03$ \\
\hline Pb208 & 52.4 & $1.5840 \mathrm{E}-02$ & 58.599 & 1.7706E-02 & 37.640 & 1.1395E-02 \\
\hline
\end{tabular}




\subsubsection{Neutronics Discussion}

The lead coolant composition was changed to reflect the high quality (Canon Diablo, Arizona) and low quality (Ace Mine, Saskatchewan) compositions in Table 2.10.1. The nominal density of $10.402 \mathrm{~g} / \mathrm{cc}$ was used for the high quality and low quality cases. Heterogeneously modeled regions (namely the active core region) directly used the new lead materials, and homogeneous compositions were re-homogenized using the perturbed lead materials. Table 2.10.2 summarizes the impact of variations in lead coolant properties on the reactor k-effective.

Table 2.10.2. Impact of lead coolant properties on k-effective

\begin{tabular}{|l|c|c|c|}
\hline & $\begin{array}{c}\text { Nominal } \\
\text { Condition }\end{array}$ & High Quality & Low Quality \\
\hline Density (g/cc) & 10.402 & 10.402 & 10.402 \\
\hline Pb-208 wt\% & $52.4 \%$ & $58.599 \%$ & $37.640 \%$ \\
\hline Pb-208 density (at/b-cm) & $1.5840 \mathrm{E}-02$ & $1.7706 \mathrm{E}-02$ & $1.1395 \mathrm{E}-02$ \\
\hline k-effective & 1.17701 & 1.17898 & 1.17034 \\
\hline Delta-k (pcm) from Nominal & - & 197 & -667 \\
\hline
\end{tabular}

The high quality lead contains $58.588 \% \mathrm{~Pb}-208$ (slightly more $\mathrm{Pb}-208$ by weight than the nominal case). Using high quality lead coolant increases k-eff by $+197 \mathrm{pcm}$ over the nominal case. The use of high quality lead could present an opportunity to reduce enrichment of the fuel. Since high quality lead contains a larger proportion of the $\mathrm{Pb}-208$ isotope than the "nominal" lead composition, fewer neutrons are lost due to interactions with the lead coolant.

The low quality lead contains $37.640 \% \mathrm{~Pb}-208$ (much less $\mathrm{Pb}-208$ than the nominal case) in which less favorable lead isotopes (from a neutron economy perspective) are traded for the missing $\mathrm{Pb}-208$. The use of low quality lead reduces the k-effective by $667 \mathrm{pcm}$ due to additional neutron- $\mathrm{Pb}$ interactions which prevent some neutrons from inducing fission.

Despite large impact on eigenvalue, the power distribution did not change appreciably because the power was held constant in the assembly. As shown in Figure 2.10.1, the integrated pin powers vary by less than $0.05 \%$ from the nominal condition which is insignificant in terms of computing hot spots on the pin. Figure 2.10.2 shows that the high quality and low quality compositions do impact the axial power distribution by a very small amount, shifting more power to the center of the active core in the low quality case. The figure displays the data for the center pin, and the same behavior is present in the outer ring of pins. However, the change from nominal is exceedingly small $(<0.15 \%$ at any given axial plane) and would not impact the location and temperature of the hottest pin. The nominal power distribution can therefore be used for the thermal hydraulics calculation. 


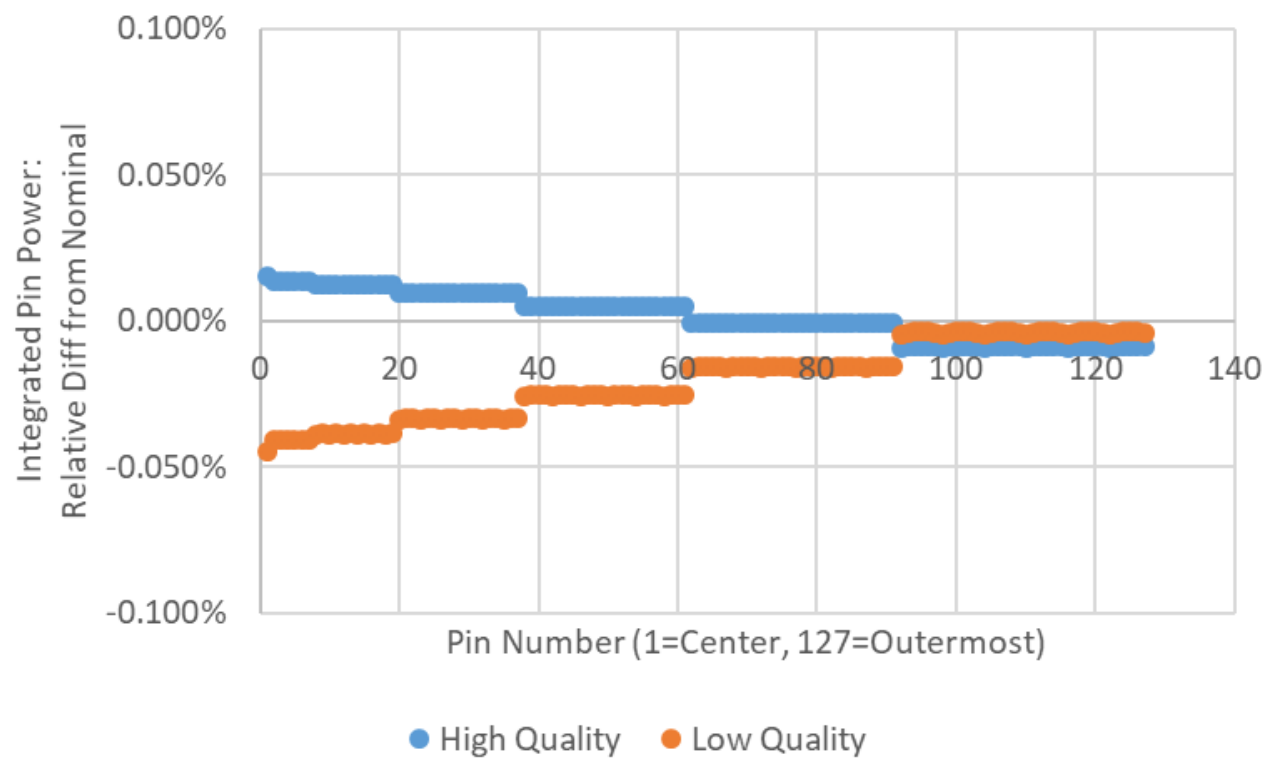

Figure 2.10.1 Relative difference in integrated pin power (high/low quality lead cases vs. nominal)

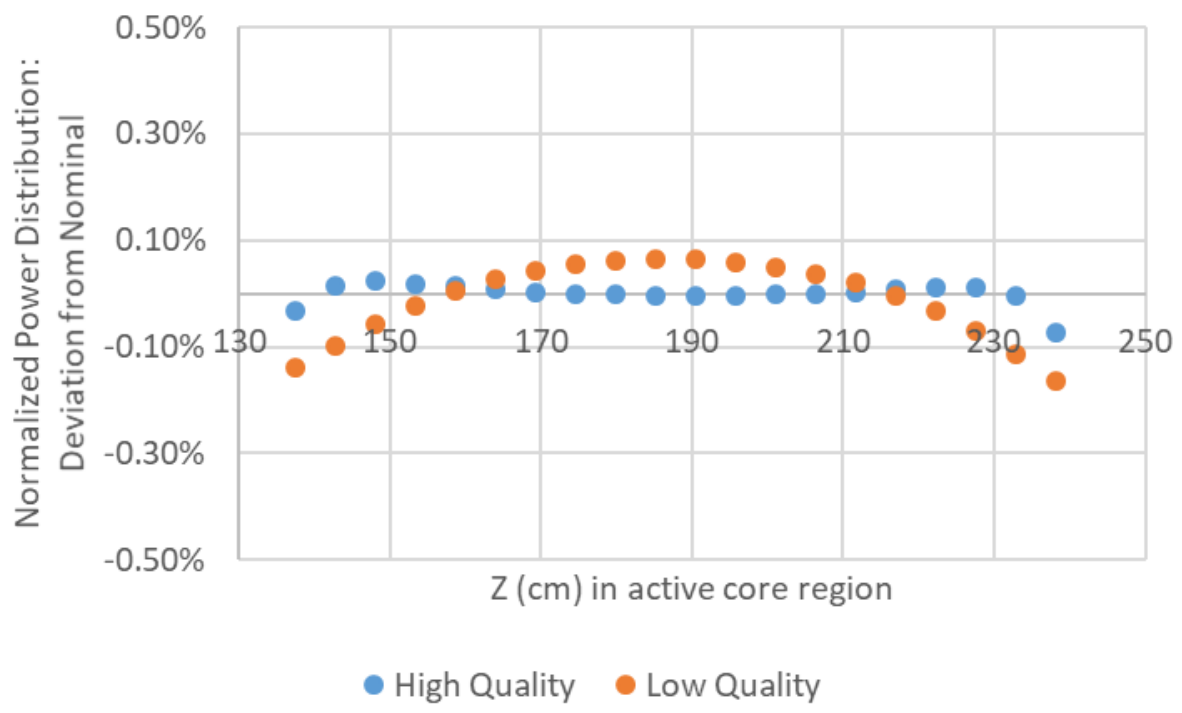

Figure 2.10.2 Relative difference in normalized axial power, center pin (high/low quality lead cases vs. nominal) 


\subsubsection{Thermal Hydraulics Discussion}

No additional thermal hydraulic calculations were needed because the impact of lead composition uncertainty did not impact the power distribution nor does it carry perturbations in thermal hydraulics parameters. Therefore, the conclusion is that the impact of lead composition on power and temperature distributions is negligible (in the case of fixed assembly power as performed here).

\subsection{HCF Associated with Uncertainties in Cladding Thermal Conductivity}

No neutronics simulations were necessary to examine the impact of uncertainties in cladding thermal conductivity since this is purely thermal hydraulic parameter. Therefore, the nominal condition power solution was used for the thermal hydraulic analysis of this hot channel factor. Since the cladding is mainly made of stainless steel, the cladding thermal conductivity is assumed to be $21.6 \mathrm{~W} / \mathrm{m} \cdot \mathrm{k}$ with physical uncertainty of $10 \%$ [Ho 1977]. The uncertainty of cladding thermal conductivity can increase the temperature gradient in cladding, which leads to higher maximum cladding temperature. The cladding thermal conductivity was modified directly in the code performing the calculations; by doing so, the effects of uncertainties are properly taken into account.

The maximum cladding temperature of the pins at the outmost ring (pin number: 92 127) are shown in Figure 2.11.1. The peaks of the profiles indicate the maximum cladding temperature of the pins in the corner. The perturbed cladding thermal conductivity will influence thermal conduction in the radial direction. The impact of the perturbed cladding thermal conductivity on the axial and circumferential conduction are also taken into account in 3D simulation. The results show that the impact of cladding thermal conductivity on each pin is about $3 \sim 4 \mathrm{~K}$. 


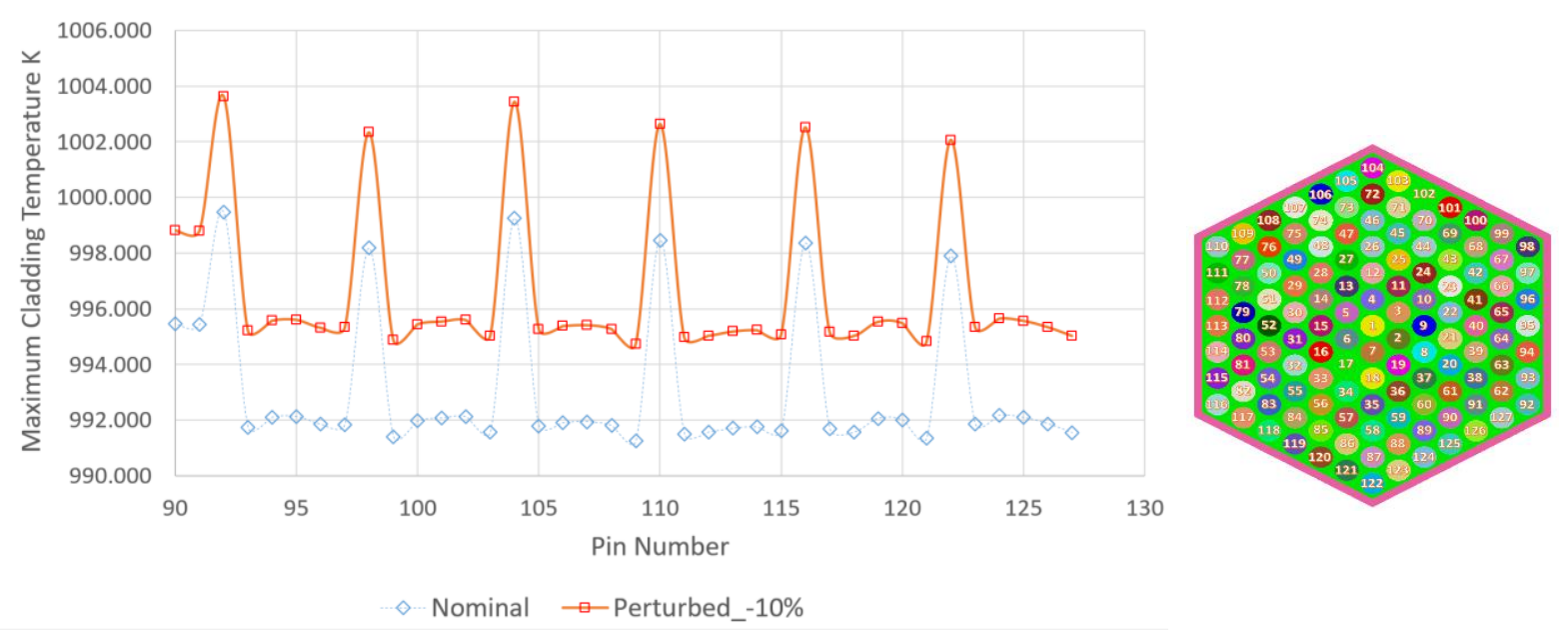

Figure 2.11.1 Maximum cladding temperature responding to the uncertainty of cladding thermal conductivity

\subsection{HCF Associated with Uncertainties in Fuel Thermal Conductivity}

No neutronics simulations were necessary to examine the impact of uncertainties in fuel thermal conductivity since this is a purely thermal hydraulic parameter. Therefore, the nominal condition power solution was used for the thermal hydraulic analysis of this hot channel factor. The fuel burnup, fuel chemistry evolution and fuel swelling will all have impact on the fuel thermal conductivity. The fuel thermal conductivity is assumed to be $1.882 \mathrm{~W} / \mathrm{m} \cdot \mathrm{k}$ with physical uncertainty of $21.3 \%$ [Grudzinski 2019]. The uncertainty of fuel thermal conductivity can increase the temperature gradient in fuel, which leads to higher maximum fuel temperature. The cladding thermal conductivity was modified directly in the code performing the calculations; by doing so, the effects of uncertainties are properly taken into account.

\subsection{HCF Associated with Uncertainties in Gap Conductance}

At the initial fresh fuel condition, there is a sizable helium gap between the fuel pellet and cladding. This gap allows for fuel expansion due to thermal and irradiation-induced swelling. As the fuel burns up (around 8\%) [Rineiskii 1999], fission products are generated in the fuel and some of these byproducts migrate preferentially towards the fuel pellet edge due to high volatility and strong temperature gradient [Smith 2017]. In the high-burnup fuel rod, the solid fission product oxide deposit builds up in the gap between the fuel pellet and the cladding, a major composition of which is cesium molybdenate $8 \sim \mathrm{Cs} 2 \mathrm{MoO} 4$. This deposit is called "joint oxide gain (JOG)", but the specific mechanism of its formation is not yet understood. JOG has an effect of decreasing the fuel swelling and improving the gap conductance. The decrease of fuel swelling is expected to prevent the excessive Fuel-Cladding Mechanical Interaction (FCMI), and the improvement of the gap conductance could lead to lower fuel center temperature. Therefore, it 
is very important to estimate the effect of JOG formation in the thermal computation. Figure 2.13.1 shows the structure of JOG in the open literature [Melis 1993][Cappia 2020]. The JOG formation does not smoothly contact the cladding in reality but has jagged edges. The conductance of the JOG vs. the gap is a subject of interest when computing the fuel temperature.

JOG grows regularly with increasing burn-up. JOG contains mainly $\mathrm{Mo}, \mathrm{Cs}, \mathrm{O}$ with frequent presence of $\mathrm{Ba}, \mathrm{Te}, \mathrm{Pd}$ [Melis 1993]. The thermal conductivity of the JOG material is of particular interest since it is the main input parameter in any fuel-clad gap conductance calculation. Moreover, clear understanding of the impact of the JOG layer is of potential importance to fuel behavior at high burn-up. The gap conductance, mainly in the case of cladding swelling, is dependent not only on fission gas conductance, but also on the fission product conductivity and JOG size. The JOG size depends on many factors such as temperature, pressure, burn-up and fission gas release.

In Figure 2.13.2, a study [Grudzinski 2019] using SAS4A qualitatively demonstrates multiple phenomena taking place to drive the gap conductance. A reactor starts from the zero power level and ramps up. The gas gap thickness quickly goes down at early burnup, and the gap conductance is enhanced due to fuel expansion. Then the gap conductance comes down gradually due to fission gas release and gas thermal conductivity degradation (fission gas to helium ratio increases). Once the JOG starts forming, it fills the gap quickly and enhances the gap conductance. Without JOG effect, the degradation of gap conductance would be very significant at high burnup due to fission gas release. The JOG size increases with burn-up as cesium and molybdenum release from the fuel continues.

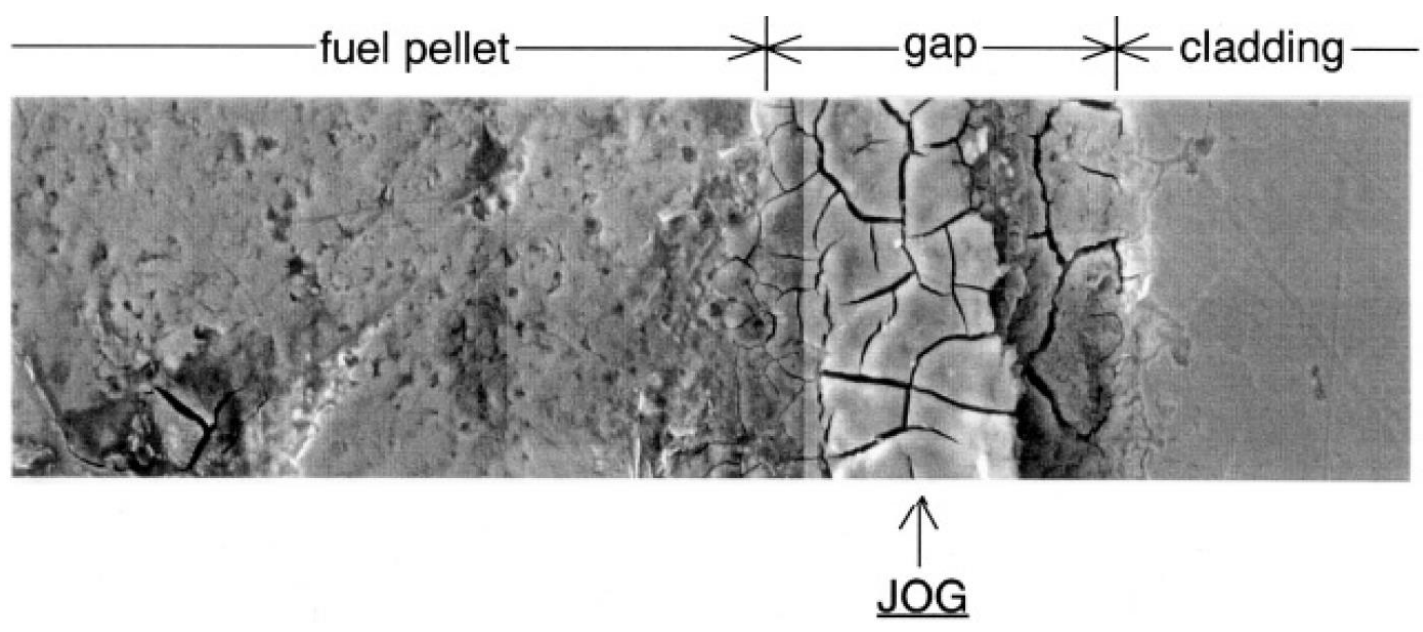




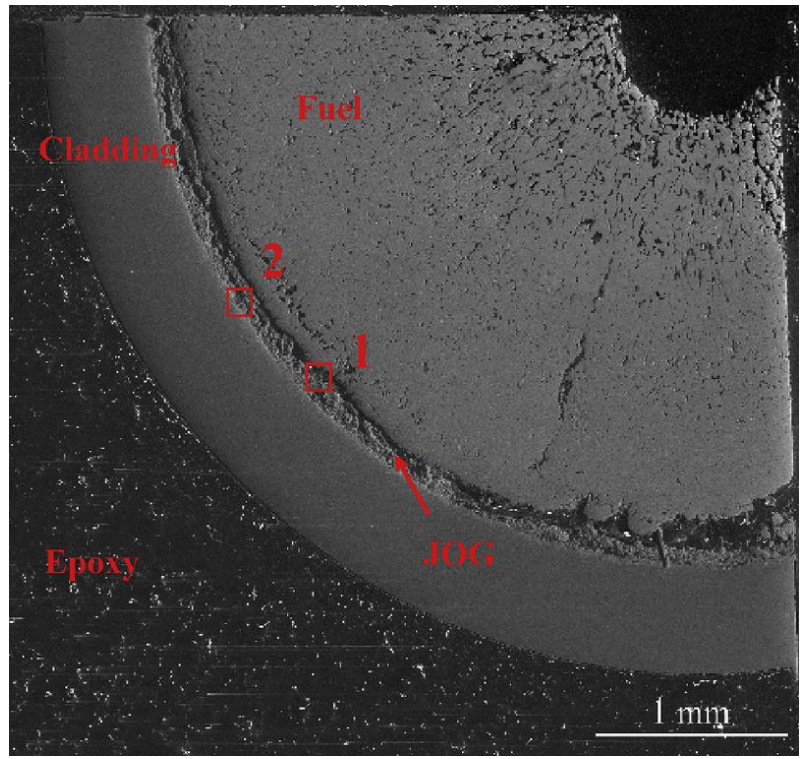

Figure 2.13.1 JOG formation in pellet-cladding gap

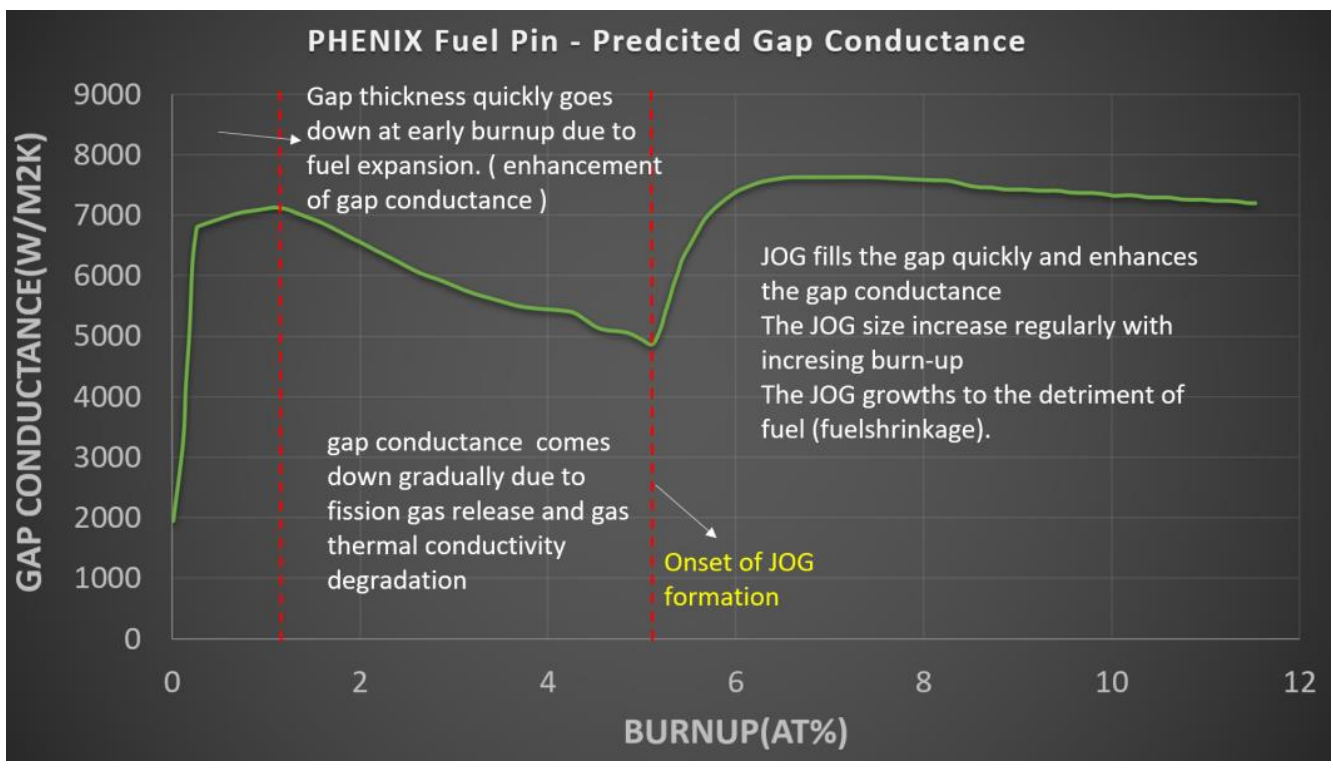

Figure 2.13.2 Gap conductance change along with the increase of burnup

Given limited information on the formation of joint oxide gain (JOG), the two bounding conditions are compared: (1) nominal condition, where fuel is fresh and there is a fully open fuel-cladding gap with no JOG formation, and (2) perturbed condition, where fuel is burned and the fuel-cladding gap is assumed to be completely closed due to JOG formation. 


\subsubsection{Neutronics Discussion}

In neutronics, simulation of the perturbed condition requires the burned fuel composition, which was provided by Westinghouse at $41 \mathrm{MWDd} / \mathrm{kgU}$ burnup. The true gap size is unknown at this burnup, but assumed to be closed. In the neutronics model, the neutron physics is similar whether the gap is open or closed provided the fuel mass is preserved. Therefore, the original mesh with open gap was used to simulate this burned case, and a burned fuel density resembling the original density was used. The effect of the depleted fuel composition on the power shape and magnitudes was observed compared to the nominal condition.

The eigenvalue for the burned case is 1.10391 compared to the fresh fuel case of 1.17697. Figure 2.13.3 shows the nominal and burned axial power profiles in the center pin of the LFR fuel assembly. Increased power $(+6 \%)$ is observed at the top and bottom of the active core which changes the profile slightly from the nominal condition. The middle of the core power is slightly suppressed (-1.7\%). The maximum pin power in the burned case is $28.80 \mathrm{~kW}(+40 \mathrm{~W}$ from nominal condition), located in the same corner pins as the nominal condition. Therefore, the burned power distribution does exhibit differences compared to the nominal condition.

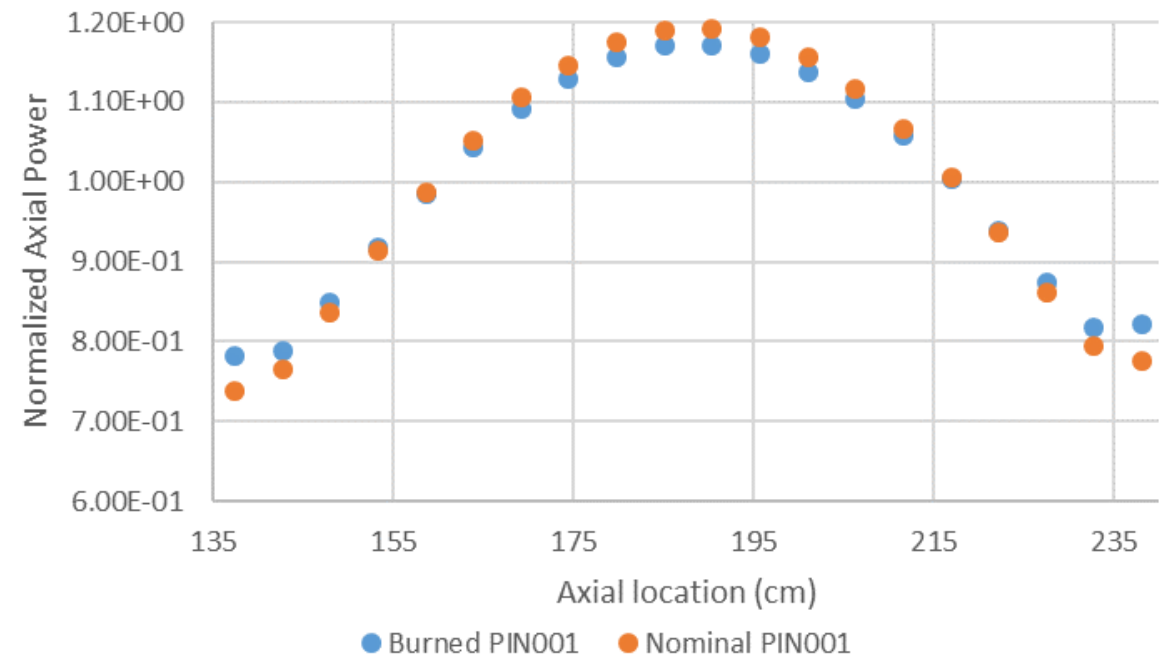

Figure 2.13.3 Nominal and burned axial power profile (center pin)

The burned power distribution from PROTEUS is used in Nek5000 for simulation of the closed gap condition. 


\subsubsection{Thermal Hydraulics Discussion}

Since the major composition of JOG is $\mathrm{Cs}_{2} \mathrm{MoO}_{4}$, the thermal conductivity of JOG is established by that of $\mathrm{Cs}_{2} \mathrm{MoO}_{4}$ as in the following equation valid for the given porosity range [Ikusawa 2017][Ishii 1997]:

$$
\begin{gathered}
K_{J O G}=\frac{1-1.848 \cdot P}{0.895} \times\left(\frac{132.56}{T_{\text {gap }}}+0.03+3.2 \times 10^{-10} \cdot T_{\text {gap }}{ }^{3}\right) \\
K_{J O G}=\text { thermal conductivity of JOG } \\
P=\text { porosity of JOG }(5.7 \% \leq \mathrm{P} \leq 17.4 \%) \\
T_{\text {gap }}=\text { gap temperature }
\end{gathered}
$$

Figure 2.13.1 shows that the JOG with relatively small porosity is expected to have filled the fuel-cladding gap during irradiation. Eq. 2.13.1 is a simplified expression to compute the conductance of some specific type of JOG compound.

As shown in Figure 2.13.4, JOG growth is non-uniform in the axial direction, which causes the gap to be non-uniformly closed (zigzag shape). However, in CFD modeling, the shape of the gap is simplified as a centrosymmetric cylinder. The gap thickness can change from zero (fully closed) to fully open. The gap conductance depends not only on the fission gas conductance but also on the JOG size (Figure 2.13.5). 


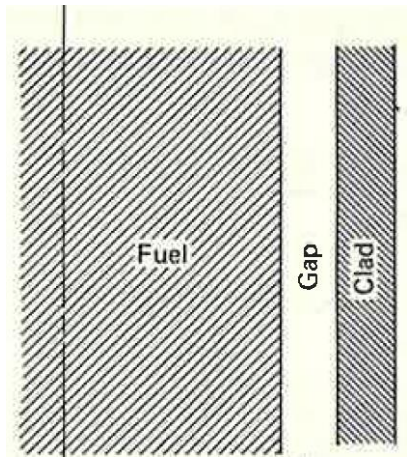

Prior to irradiation

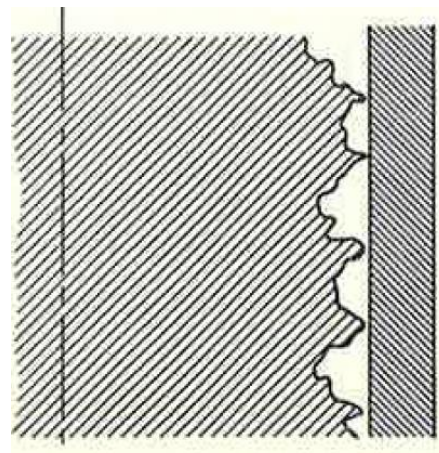

After irradiation

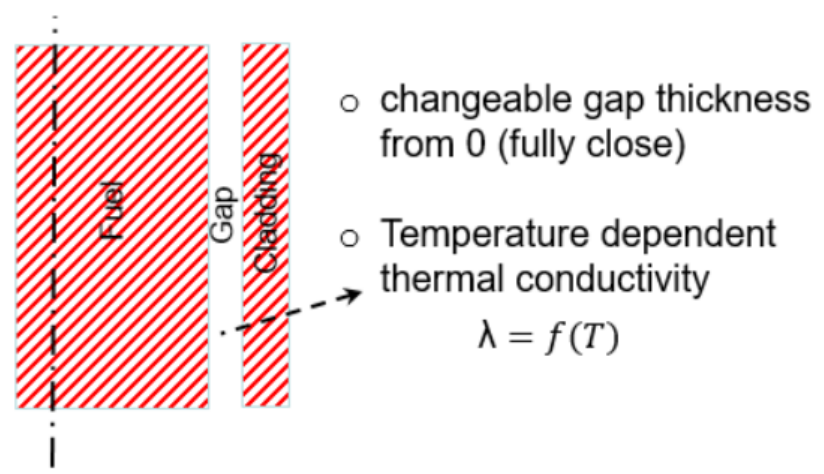

Figure 2.13.4 The effect of irradiation on the fuel-clad gap (top) and CFD model (bottom)

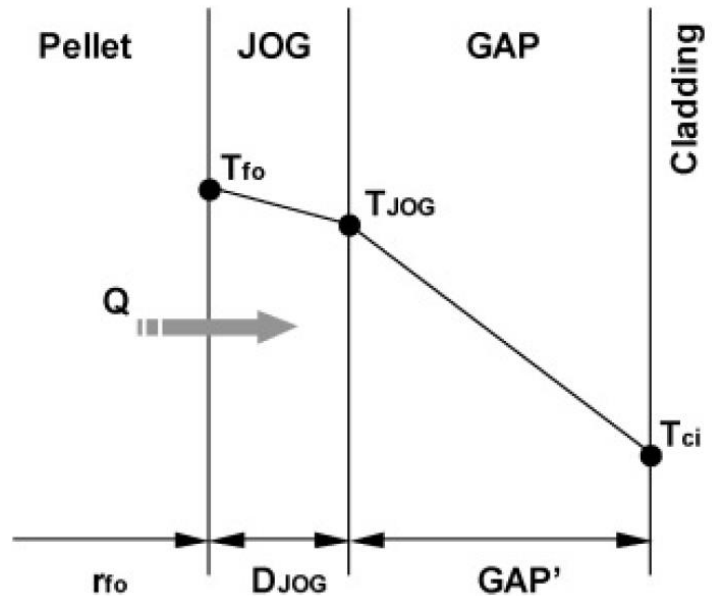

Figure 2.13.5 Outline of thermal computation in the pellet-cladding gap with JOG 
When the gap is fractionally filled with JOG, the gap conductance can be calculated from the following equation:

$$
H_{G A P}=\frac{h_{G A P} \cdot h_{J O G}}{h_{G A P I}+h_{J O G}}
$$

$$
\begin{gathered}
H_{G A P}=\text { gap conductance with an effect of JOG } \\
h_{G A P,}=\text { gap conductance of mixed gas in a gap of size GAP' } \\
h_{J O G}=\text { gap conductance of } J O G
\end{gathered}
$$

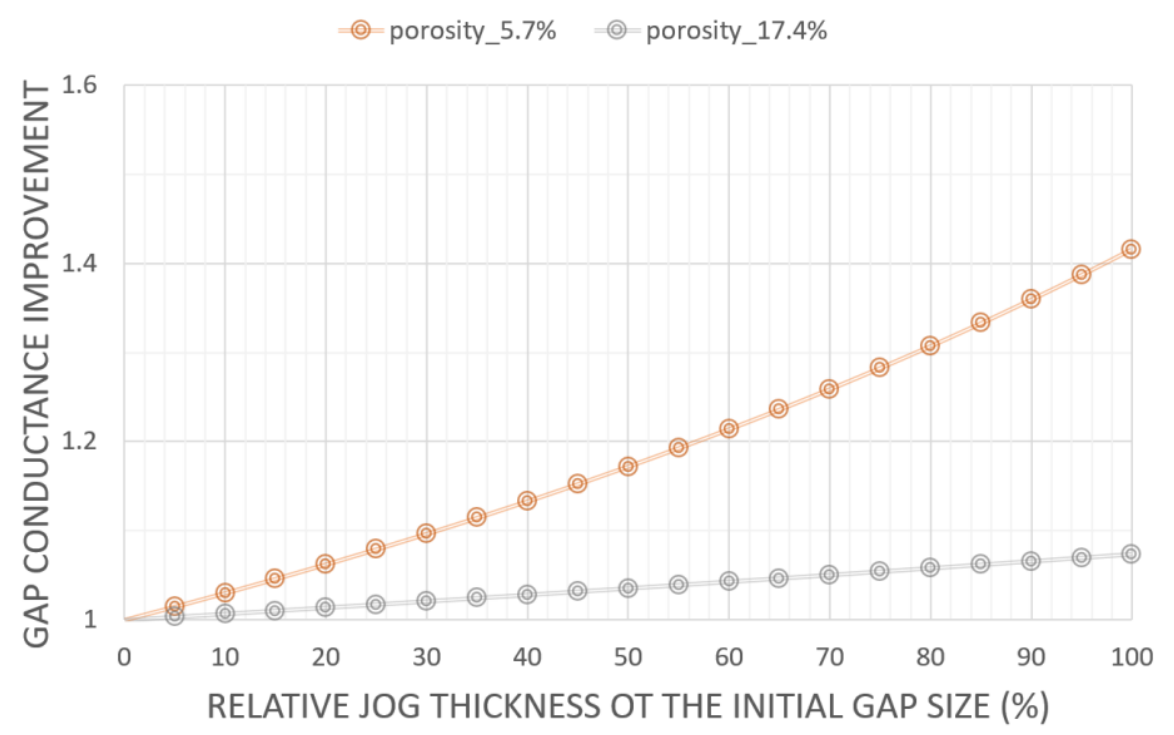

Figure 2.13.6 Gap conductance improvement due to the JOG formation

In Figure 2.13.6, the relative improvement in gap conductance (compared to a case with no JOG formation) is plotted against the relative JOG thickness within the gap. When the gap is fully filled with JOG (relative JOG thickness of the initial gap size $=100 \%$ ), the JOG with different porosity show different gap conductance improvement. JOG with $5.7 \%$ porosity improves the gap conductance by $1.4 \mathrm{x}$, while JOG with $17.4 \%$ porosity only improves the gap conductance by $1.07 x$. That is simply because the JOG with lower porosity has higher thermal conductivity. In addition, the experimental study of thermal gap and contact conductance between depleted uranium dioxide (UO2) and Zircaloy-4 (Zr4) [Garnier 1979] shows that the analytical expressions for ideal gap conductance (such as Eq. 2.13.1) underestimates the gap conductance. For instance, the maximum gap conductance measured in the experiment is 25 times higher. Generally, the experimental data covered a wide range of conditions and showed up to 18-fold factor difference in gap conductance under different circumstance. It indicates that the gap conductance is highly dependent on pressure, temperature and gas composition. Simply determining the gap conductance with porosity may introduce uncertainties. It is necessary to estimate of the gap conductance through experimental research and/or advanced fuels modeling. 
Table 2.13.1 shows the temperature rise with different gap conductance from expression and experiment for a "fully closed" gap with JOG. The JOG formation improves the gap conductance, which is beneficial for reactor design in terms of fuel temperature reduction. In terms of the cladding temperature rise, the impact of gap conductance is slight. However, the gap conductance has significant impact on maximum gap and fuel temperature. This study demonstrates the significance of the understanding on JOG formation mechanism and accurate estimation of gap conductance.

Table 2.13.1 Temperature rise with different gap conductance

\begin{tabular}{cccc}
\hline Gap Conductance & $\Delta \mathrm{T}$ _Cladding & $\Delta \mathrm{T}_{-}$Gap & $\Delta \mathrm{T}_{-}$Fuel \\
\hline $\begin{array}{c}\text { Nominal } \\
\text { (gap fully open) }\end{array}$ & 28.62 & 285.18 & 1175.36 \\
\hline $\begin{array}{c}\text { Perturbed expression } \\
\text { (gap fully closed) }\end{array}$ & 28.62 & 205.77 & 887.23 \\
\hline $\begin{array}{c}\text { Perturbed } \\
\text { experiment } \\
\text { (gap fully closed) }\end{array}$ & 28.47 & 5.061 & 749.65 \\
\hline
\end{tabular}

\subsection{HCF Evaluation and Summary}

First-of-a-kind high fidelity hot channel factor calculations were carried out for a lead-cooled fast reactor design during FY19 and FY20. Specifically, HCFs for perturbed coolant properties, thermal conductivities, and manufacturing tolerances were evaluated for Westinghouse's LFR design (inner zone assembly) with the SHARP toolkit (PROTEUS/Nek5000). The results are listed in Table 2.14.1 along with the HCF estimation for AFR-100 computed by SHARP in FY18 and the legacy HCF data for EBR-II. The uncertainty in LFR parameters were taken from SFR data (cladding thickness) and from discussion with Westinghouse. Code to code comparison, sensitivity studies, and model selection were performed to improve the reliability of the results. No attempt is made to fit the data to EBR-II data since most HCF data for EBR-II comes from low fidelity model or lumped parameter method.

The cladding thickness calculation was based on direct calculations where the cladding thickness was changed in all pins by the maximal and minimal amounts. The high fidelity simulation accounts simultaneously for the impact of cladding thickness on heat transfer in the cladding as well as the flow area around each pin. Localized temperature impacts were found that were non-intuitive. Due to different pin and assembly dimensions and materials, the impact of cladding thickness manufacturing tolerance has a larger impact on the peak cladding temperature for the LFR design than for the AFR-100. 
The hot channel factor from manufacturing fissile mal-distribution was evaluated based on a stochastic technique. Thirty fuel assembly models were randomly generated according assuming a normal distribution in pin $\mathrm{Pu}-239$ content. The models were simulated in PROTEUS, and the resulting power distributions were provided to Nek5000 for temperature calculations. The manufacturing tolerance and fuel type was different in the LFR design (MOX fuel vs metallic fuel in EBR-II and AFR-100). Such differences make it difficult to compare and apply hot channel factors across reactor types, however, the LFR HCF was in the (somewhat wide) range of those observed for SFRs.

The HCF calculation for material properties such as coolant specific heat, coolant density, cladding thermal conductivity and fuel thermal conductivity were performed. For those calculations, either no neutronics calculations were necessary or the impact of the material properties are negligible on power solution. Therefore, the nominal condition power solution was used for those hot channel factor calculation. They are all based on a direct calculation where the coolant specific heat property was perturbed by $-5 \%$, the coolant density was perturbed by $0.8 \%$, the cladding thermal conductivity was perturbed by $-10 \%$ and the fuel thermal conductivity was perturbed by $-21.3 \%$. The larger uncertainties lead to a larger hot channel factor for LFR than seen in the SFR designs and vice versa. The coolant specific heat calculation was based on a direct calculation where the coolant specific heat property was perturbed by $-5 \%$. The larger uncertainty in lead specific heat caused a larger hot channel factor for LFR than seen in the SFR designs. The coolant density calculation was also based on a direct calculation where the density was reduced by $0.8 \%$, which is a slightly larger perturbation than assumed for sodium coolant in the AFR-100 and EBR-II calculations. Nevertheless, the perturbation resulted in a coolant HCF identical to the AFR-100 SFR design (1.001). The cladding thermal conductivity uncertainty was larger than for SFR designs, and the resulting cladding HCF was larger as well. The fuel thermal conductivity was lower than that of SFR designs, and the resulting fuel HCF was lower too. However, without detailed simulation of the precise design, the impact of uncertainties on the HCF cannot be pre-determined simply based on magnitude.

The coolant isotopic uncertainty was based on an assumption of lead coming from different origin mines - creating worst and best case scenarios in terms of $\mathrm{Pb}-208$ content. The low quality lead and high quality lead did impact the eigenvalue but did not impact the power distributions (the assembly was kept at constant power) appreciably enough to create differences in a thermal hydraulics calculation, so these were not performed.

The study on gap conductance consisted of simulations at fresh condition (open gap, fresh fuel) and at burned condition (closed gap, burned fuel) to estimate the bounding cases. Literature correlations were referenced to compare gap conductance models. The formation of JOG was shown to be beneficial for reactor design in term of fuel temperature reduction.

Table 2.14.1 also demonstrates the usefulness of performing detailed HCF analysis for different reactor designs. First, the uncertainties are different for different reactor. Second, the 
impact of these uncertainties are different as well. For instance, the HCF of coolant specific heat for LFR are higher than that for SFR because the density of lead is almost 10 times higher than that of sodium, and because the uncertainty is higher for lead. It is not recommended to use HCF from a different reactor type since the data may not be conservative. This procedure of computing HCF using high fidelity models shows promise for being repeated for any arbitrary reactor of choice, since these tools are targeted to solve many reactor types and geometries. 
Table 2.14.1 HCF Estimation for EBRII, AFR-100, and LFR

\begin{tabular}{|c|c|c|c|c|c|c|c|c|c|c|c|c|}
\hline \multirow{2}{*}{ HCF } & \multicolumn{3}{|c|}{ Uncertainties $(3 \sigma) \%$} & \multicolumn{3}{|c|}{$\begin{array}{c}\text { Coolant HCF } \\
\text { (A) }\end{array}$} & \multicolumn{3}{|c|}{$\begin{array}{c}\text { Cladding HCF } \\
\text { (B) }\end{array}$} & \multicolumn{3}{|c|}{$\begin{array}{l}\text { Fuel HCF } \\
\text { (C) }\end{array}$} \\
\hline & EBRII & $\begin{array}{l}\text { AFR- } \\
100\end{array}$ & LFR & EBRII & $\begin{array}{l}\text { AFR- } \\
100\end{array}$ & LFR & EBRII & $\begin{array}{l}\text { AFR- } \\
100\end{array}$ & LFR & $\begin{array}{c}\text { EBRI } \\
\text { I }\end{array}$ & $\begin{array}{l}\text { AFR- } \\
100\end{array}$ & LFR \\
\hline $\begin{array}{l}\text { Cladding } \\
\text { Thickness } \\
\text { (mm) }\end{array}$ & \pm 0.05 & \pm 0.05 & \pm 0.05 & & & & $\begin{array}{c}1.03^{\sim} \\
1.05\end{array}$ & 1.02 & 1.05 & & & \\
\hline $\begin{array}{c}\text { Coolant } \\
\text { Specific } \\
\text { Heat }(\mathrm{J} / \mathrm{kg} \cdot \mathrm{K})\end{array}$ & \pm 3 & \pm 3 & \pm 5 & 1.017 & 1.016 & 1.04 & & & & & & \\
\hline $\begin{array}{l}\text { Fissile Mal- } \\
\text { distribution }\end{array}$ & \pm 6 & \pm 6 & \pm 5 & & & & 1.06 & 1.04 & 1.04 & 1.06 & 1.02 & 1.04 \\
\hline $\begin{array}{l}\text { Coolant } \\
\text { Density } \\
\left(\mathrm{kg} / \mathrm{m}^{3}\right) \\
\end{array}$ & \pm 0.5 & \pm 0.5 & \pm 0.8 & 1.016 & 1.001 & 1.001 & & & & & & \\
\hline $\begin{array}{c}\text { Cladding } \\
\text { Thermal } \\
\text { Conductivity } \\
(\mathrm{W} / \mathrm{m} \cdot \mathrm{K})\end{array}$ & \pm 7 & \pm 7 & \pm 10 & & & & 1.088 & 1.082 & 1.113 & & & \\
\hline $\begin{array}{c}\text { Fuel } \\
\text { Thermal } \\
\text { Conductivity } \\
(\mathrm{W} / \mathrm{m} \cdot \mathrm{K})\end{array}$ & \pm 25 & \pm 25 & \pm 21.3 & & & & & & & 1.25 & 1.23 & 1.17 \\
\hline
\end{tabular}

Table 2.14.2 Evaluation of LFR temperature rises (degrees K) in hot channels

\begin{tabular}{|c|c|c|c|c|c|c|c|}
\hline \multirow{2}{*}{ HCF } & \multirow{2}{*}{$\begin{array}{c}\text { Uncertainties } \\
(\%)\end{array}$} & \multicolumn{2}{|c|}{$\begin{array}{c}\text { Coolant HCF } \\
\text { (A) }\end{array}$} & \multicolumn{2}{|c|}{$\begin{array}{c}\text { Cladding HCF } \\
\text { (B) }\end{array}$} & \multicolumn{2}{|c|}{$\begin{array}{l}\text { Fuel HCF } \\
\text { (C) }\end{array}$} \\
\hline & & $\Delta \mathrm{T}_{\mathrm{N}}$ & $\Delta T_{p}$ & $\Delta \mathrm{T}_{\mathrm{N}}$ & $\Delta \mathrm{T}_{\mathrm{P}}$ & $\Delta \mathrm{T}_{\mathrm{N}}$ & $\Delta \mathrm{T}_{\mathrm{P}}$ \\
\hline Cladding Thickness & $\pm 0.05 \mathrm{~mm}$ & & & 28.62 & 29.93 & & \\
\hline Coolant Specific Heat & \pm 5 & 278.79 & 289.94 & & & & \\
\hline Fissile Mal-distribution & \pm 5 & & & 28.62 & 29.73 & 1305.6 & 1357.3 \\
\hline Coolant Density & \pm 0.8 & 278.79 & 279.07 & & & & \\
\hline $\begin{array}{l}\text { Cladding Thermal } \\
\text { Conductivity }\end{array}$ & \pm 10 & & & 28.62 & 31.85 & & \\
\hline $\begin{array}{l}\text { Fuel Thermal } \\
\text { Conductivity }\end{array}$ & \pm 21.3 & & & & & 1305.6 & 1527.6 \\
\hline
\end{tabular}

N: Nominal P: Perturbed 
Table 2.14.3 Equations to compute coolant, cladding, and fuel hot channel factors

\begin{tabular}{|c|c|}
\hline Coolant HCF (A) & $\begin{aligned} H C F_{A}= & \left(T_{\text {max_coolant }}-T_{\text {inlet_coolant }}\right) \text { perturbed } \\
& \left(T_{\text {max_coolant }}-T_{\text {inlet_coolant }}\right) \text { nominal }\end{aligned}$ \\
\hline Cladding HCF (B) & $\begin{aligned} \mathrm{HCF}_{\mathrm{B}}= & \left(\mathrm{T}_{\text {cladding_innerwall }}-\mathrm{T}_{\text {cladding_outerwall }}\right) \text { perturbed } \\
& \left(\mathrm{T}_{\text {cladding_innerwall }}-\mathrm{T}_{\text {cladding_outerwall }}\right) \text { nominal }\end{aligned}$ \\
\hline Fuel HCF (C) & $\begin{aligned} H C F_{c}= & \left(T_{\text {fuel_centerline }}-T_{\text {fuel_outerwall }}\right)_{\text {perturbed }} / \\
& \left(T_{\text {fuel_centerline }}-T_{\text {fuel_outerwall }}\right)_{\text {nominal }}\end{aligned}$ \\
\hline
\end{tabular}




\section{Zooming Capability for the Versatile Test Reactor}

Disclaimer: All references to VTR design in this report are based on preliminary information provided by the VTR core design team. Several elements of the design such as the control assembly had not been finalized or have changed since this work was initiated. For up-to-date information on the VTR design, please contact the VTR program.

This chapter describes the progress made on demonstrating the SHARP zooming capability for the Versatile Test Reactor (VTR).

\subsection{Review of Zooming Capability}

During FY17-FY18, the SHARP zooming capability was developed and demonstrated for sodium-cooled fast reactor (SFR) applications. The SHARP zooming concept is a multiresolution modeling scheme which allows significant reduction in computational cost for fullcore simulations which provide local information. This zooming concept is useful to produce bounding values that are needed for development of licensing documents or a specific fuel development. For instance, in order to assure the integrity of fuel elements, the bounding values such as peak cladding inner-wall temperature, peak fuel centerline temperature, peak discharge burnup, peak linear power density, etc., are needed rather than the integral or average values. For development of a specific fuel concept, local irradiation information is needed rather than the integral information.

In neutronics (PROTEUS), coarse-and-homogeneous meshes and fine-and-heterogeneous meshes are combined: i.e., most assemblies are represented using the conventional homogenized meshes, but a few assemblies of interest are represented with pin-by-pin heterogeneous meshes in order to gain accurate local information. The detailed information from the heterogeneous focal region is post-processed and transferred to the thermal hydraulics code (Nek5000) which simulates a single assembly in explicit detail. Surrounding assemblies can be ignored due to sufficient insulation of individual assemblies by the assembly duct structures. This zooming procedure was demonstrated in FY18 on a fuel assembly in the AFR-100 SFR core (Figure 3.1.1) for calculating temperature distributions in a selected fuel assembly. The zooming capability was able to identify the hot spot location within the focal assembly.

Key benefits of the zooming calculation are the ability to build the neutronics model once provided each focal assembly is explicitly represented, and then the execution of $\mathrm{T} / \mathrm{H}$ simulations on multiple assemblies in parallel on a smaller geometry domain. This method enabled calculations requiring full-core neutronics to be computationally feasible in a multiphysics scenario. 

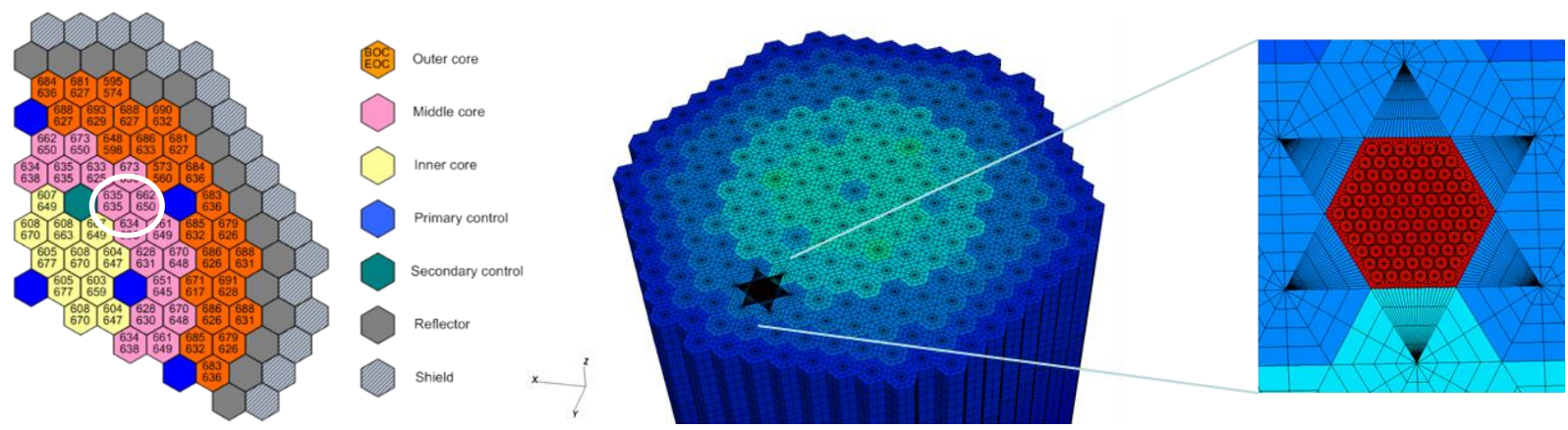

Figure 3.1.1 AFR-100 neutronics modeling for SHARP zoom calculation

\subsection{Description of VTR Zooming Objectives}

The objective of this work is to apply the multi-physics coupling and zooming capabilities to the Versatile Test Reactor (VTR) SFR design. In particular, a control assembly will be modeled and simulated by extending the previously demonstrated capabilities to a non-fueled assembly. The objective of the zooming calculations is to provide accurate control rod worth, power (gamma heating), and temperature distribution in the control assembly because the legacy tools used in the VTR design overestimate control rod worth. While the VTR control assembly design is still evolving, VTR designers were consulted in FY19 to select a single preliminary design for this analysis.

The zooming method accounts for global and local spectrum effects via full core neutronics simulation with localized geometry heterogeneity as well as heterogeneous cross section treatment. Detailed power distributions are transferred to $\mathrm{T} / \mathrm{H}$ in order to compute the detailed temperature distributions on a single assembly geometry, since the ducted geometry sufficiently insulates neighboring assemblies from each other. Development of a gamma transport capability in PROTEUS-SN was a pre-requisite to initiating this work to yield the correct heating distribution in a non-fueled assembly. A gamma transport capability was added to PROTEUS in late FY19. Gamma heating distributions within the control assembly are compared with a verification calculation (either MCNP or DIF3D/GAMSOR).

\subsection{VTR Model Description (see disclaimer at beginning of chapter)}

The VTR model used in this work was based on preliminary information provided by the VTR core design team. Several elements of the design such as the control assembly had not been finalized, but a single design was selected so that the analysis could proceed.

The VTR is a sodium-cooled fast reactor design optimized for flexible experimental control in the numerous test locations denoted in Figure 3.3.1. Due to the large number of test assembly locations (which can be empty or hold various materials to be irradiated), only 6 assembly positions are reserved for control rods. The design of the control assembly is being selected to 
optimize performance from several aspects. First, the control rod worth should be maximized since there are fewer positions available to insert control material. In order to maximize control rod worth, more control material should be packed into the assembly (closer pin pitch) yet these rods expand and swell under irradiation conditions, which can interfere with vertical motion of the inner duct within the stationary outer duct. Finally, the materials must be maintained within temperature limits.

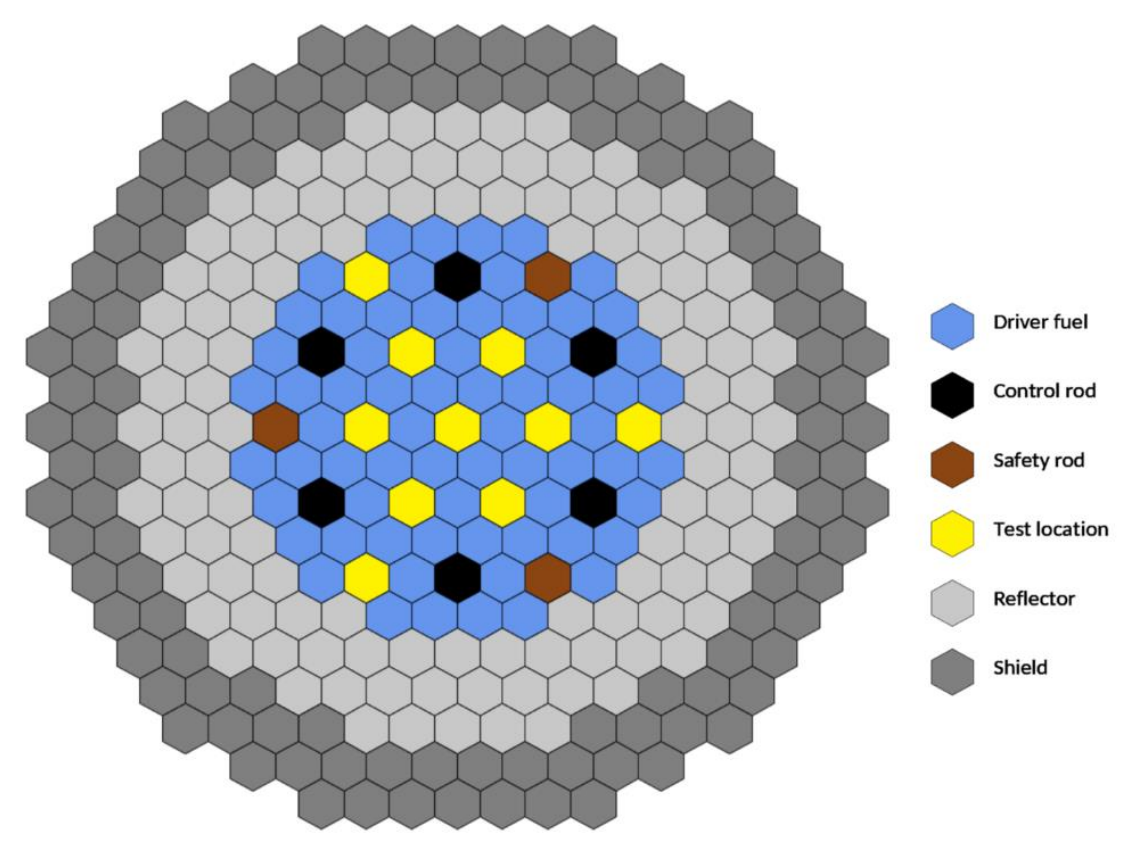

Figure 3.3.1 VTR core map 


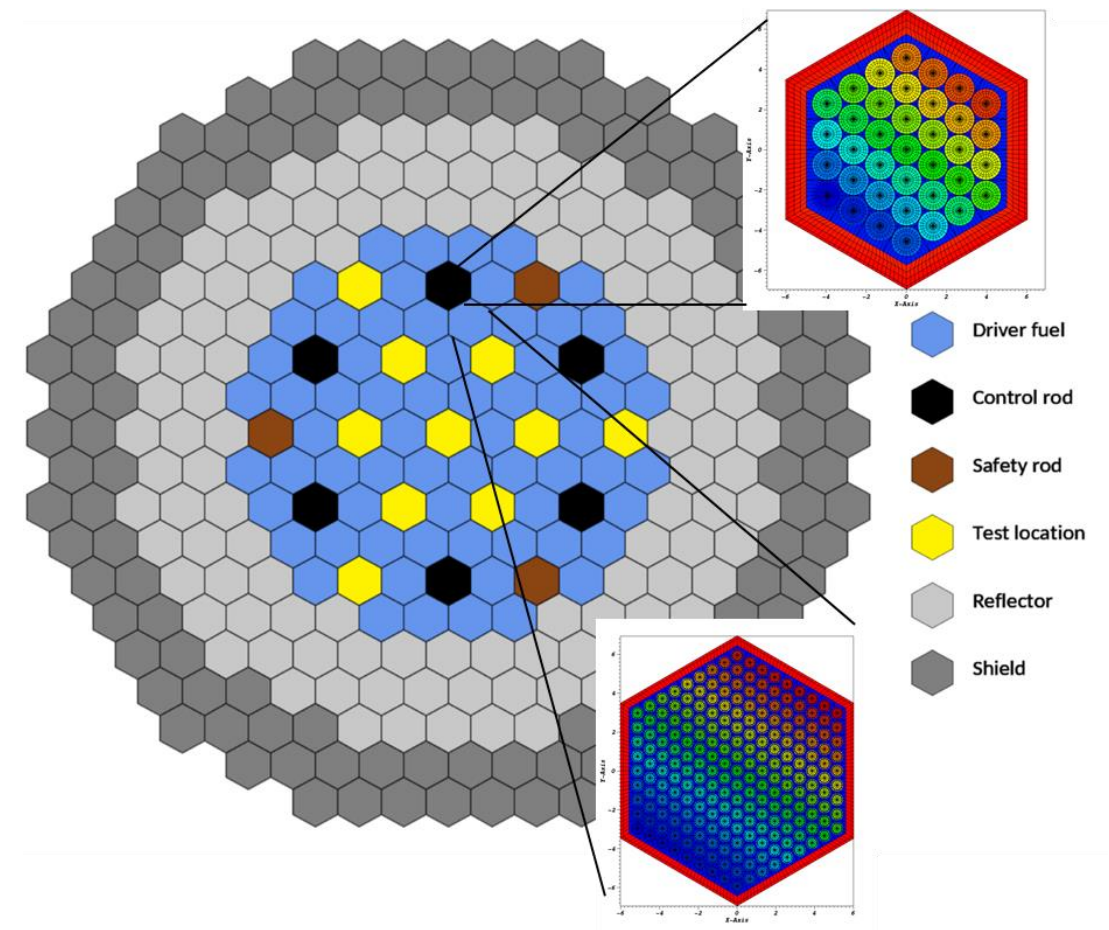

Figure 3.3.2 VTR core map showing focal assembly locations and meshes

The control assemblies are all in the 5th hexagonal ring of the core, so no individual control assembly is significantly "hotter" than the others. The primary control assemblies (control rod in figure) are surrounded by 6 fuel assemblies, while the secondary (safety rod in figure) assemblies have 5 fuel assemblies and 1 reflector assembly as neighbors. The primary control rods probably see a slightly higher neutron and gamma flux. Additionally, the primary rods are inserted in the core during operation. The primary rods will be modeled in the zooming calculation. The 6 control rods are effectively in equivalent positions around the core, so it does not matter which one is chosen for modeling, unless the flux is tilted due to asymmetric control rod positioning or material loading in the test assemblies.

Table 3.3.1 VTR control assembly parameters

\begin{tabular}{|l|c|c|}
\hline \multicolumn{1}{|c|}{ Parameter } & Cold Value & Units \\
\hline Assembly pitch & 12.0 & $\mathrm{~cm}$ \\
\hline Inter-assembly gap & 0.3 & $\mathrm{~cm}$ \\
\hline Outer duct outside flat-flat & 11.7 & $\mathrm{~cm}$ \\
\hline Outer duct inside flat-flat & 11.1 & $\mathrm{~cm}$ \\
\hline
\end{tabular}




\begin{tabular}{|l|c|c|}
\hline Outer duct thickness & 0.3 & $\mathrm{~cm}$ \\
\hline Inter-duct sodium gap thickness & 0.3 & $\mathrm{~cm}$ \\
\hline Inner duct outside flat-flat & 10.5 & $\mathrm{~cm}$ \\
\hline Inner duct inside flat-flat & 9.9 & $\mathrm{~cm}$ \\
\hline Inner duct thickness & 0.3 & $\mathrm{~cm}$ \\
\hline \# Rods & 37 & - \\
\hline P/D & 1.02231 & - \\
\hline Cladding outer radius & 0.7398 & $\mathrm{~cm}$ \\
\hline Cladding thickness & 0.0825 & $\mathrm{~cm}$ \\
\hline Helium bond thickness & 0.0514 & $\mathrm{~cm}$ \\
\hline B4C absorber radius & 0.6060 & $\mathrm{~cm}$ \\
\hline Wire wrap & Yes & - \\
\hline
\end{tabular}

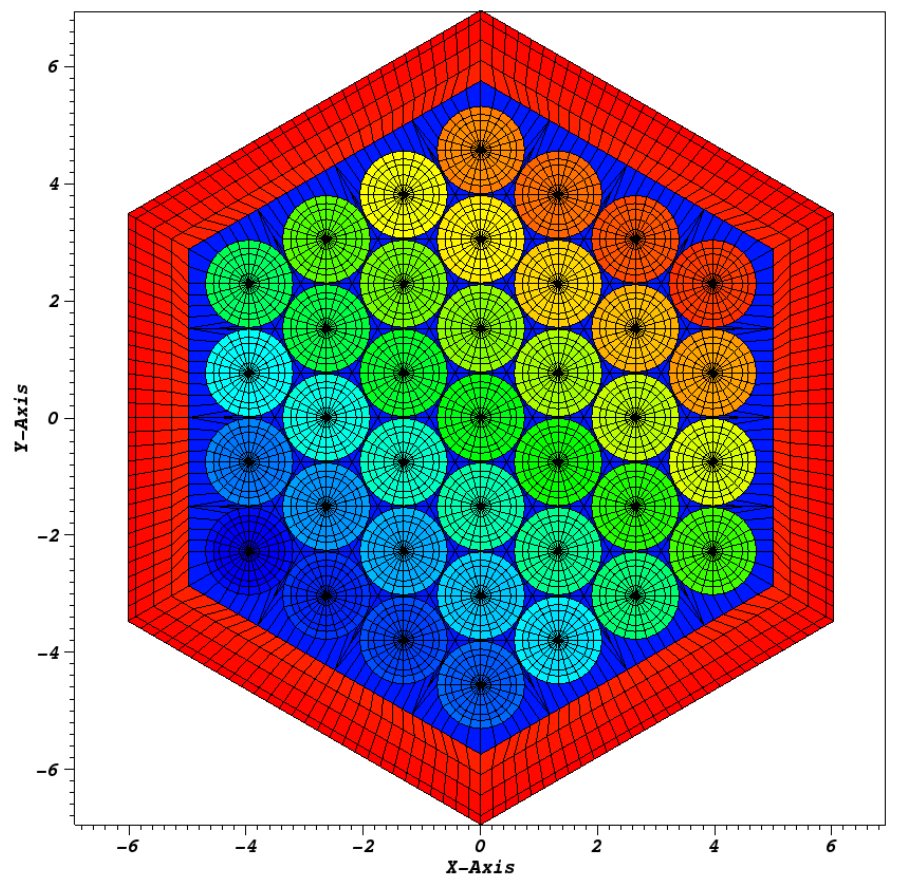

Figure 3.3.3 VTR control assembly neutronics mesh (slice)

Table 3.3.2 VTR fuel assembly parameters

\begin{tabular}{|l|l|l|}
\hline Parameter & Cold Value & Units \\
\hline
\end{tabular}




\begin{tabular}{|l|c|l|}
\hline Assembly pitch & 12.0 & $\mathrm{~cm}$ \\
\hline Duct flat-flat & 11.7 & $\mathrm{~cm}$ \\
\hline Duct thickness & 0.3 & $\mathrm{~cm}$ \\
\hline \# Rods & 217 & - \\
\hline P/D & 1.18 & - \\
\hline Cladding outer radius & 0.3125 & $\mathrm{~cm}$ \\
\hline Cladding thickness & 0.0435 & $\mathrm{~cm}$ \\
\hline Sodium bond thickness & 0.0360 & $\mathrm{~cm}$ \\
\hline Fuel slug radius & 0.2330 & $\mathrm{~cm}$ \\
\hline Wire wrap & Yes & - \\
\hline Active fuel length & 80 & $\mathrm{~cm}$ \\
\hline Fuel & $\mathrm{U}-20 \mathrm{Pu}-10 \mathrm{Zr}$ & - \\
\hline
\end{tabular}

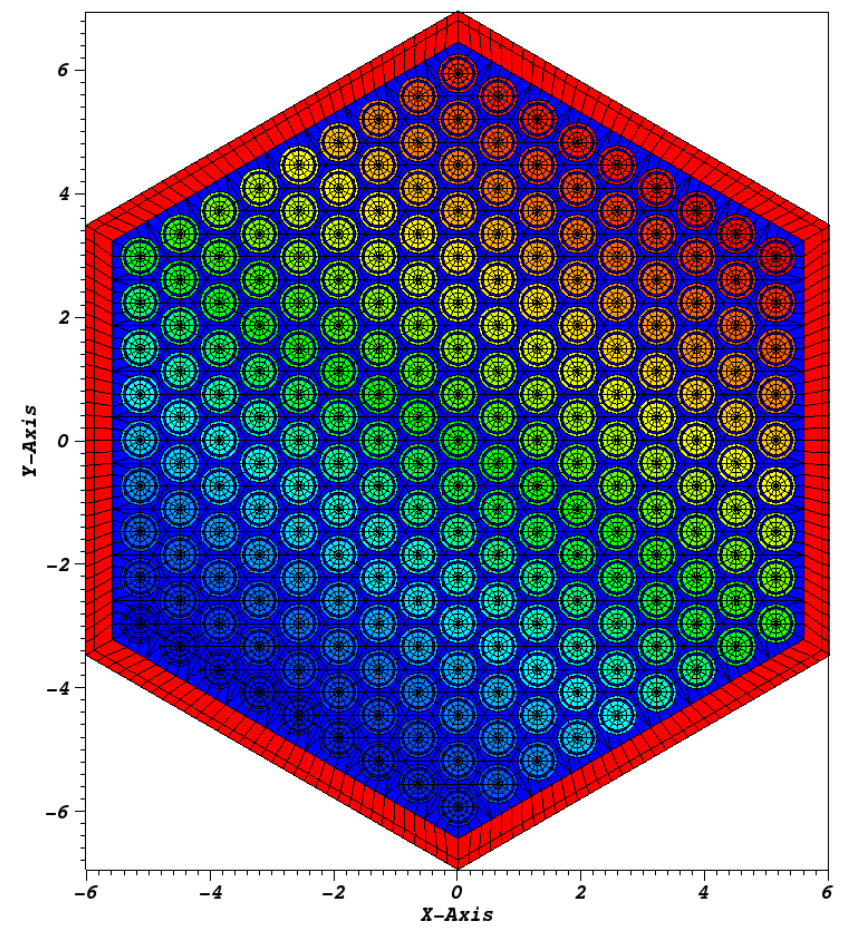

Figure 3.3.4 VTR fuel assembly neutronics mesh (slice) 
The control rods are parked $5 \mathrm{~cm}$ above the top of the active core in the "withdrawn" or "out" position. The control rods are parked $5 \mathrm{~cm}$ below the bottom of the active core in the "inserted" or "in" position. The safety rods shown in the core map are always in the "withdrawn" position.

Axial dimensions, materials, and details of the other assemblies are left out for brevity as they are not vital to the results shown here.

\subsection{Neutronics Calculations}

PROTEUS-SN was selected as the neutronics solver for the zooming calculations because it offers significant computational advantages over PROTEUS-MOCEX for large core problems with significant homogenization. This section describes the details of the neutronics calculations.

The neutronics objectives are to calculate (1) control rod worth, (2) power distribution in control assembly (control rods inserted), and (3) power distribution in fuel assembly (control rods withdrawn). The power from (2) and (3) will be transferred to Nek5000 for computation of temperature fields.

Due to the focus on control assembly physics, development of a gamma transport capability was first required. Unlike fuel assemblies where fission energy dominates the power distribution, in non-fueled assemblies, absorption reactions and other gamma producing reactions dominate the power distribution. These gamma particles transport from one region to another until they deposit heat in the medium. Gamma transport capability accounts for this movement correctly. The previous version of PROTEUS made a simplifying assumption that gamma heating is deposited at the source generation site (no transport), which properly preserves the heating amount, but not the correct distribution. Gamma transport was fully implemented in both PROTEUS-SN and PROTEUS-MOC as of June 2019. Several MCNP calculations were set up and run as verification calculations. Full core calculations with homogeneous assemblies were completed in both MCNP (including gamma transport) and PROTEUS-SN. The full core calculations with heterogeneous assemblies were attempted but not finished during FY20.

\subsubsection{Neutronics Verification Calculations}

Verification calculations were set up to compare the eigenvalues and flux distributions in PROTEUS-SN with those in MCNP6. Several cases were performed including a 2D slice of the heterogeneous fuel assembly, the 3D core in a fully homogenized configuration (control rods in and out), and the 3D core with a heterogeneous control assembly (control rods in and out). All of the desired MCNP6 calculations were completed. The 3D heterogeneous configuration cases in PROTEUS-SN were not completed yet due to difficulties debugging the large scale simulations on leadership computing resources, which will be discussed further.

The results of the verification studies in Table 3.4.1 show excellent agreement between continuous energy Monte Carlo (MCNP) and PROTEUS-SN with 33 energy groups. The differences are less than $125 \mathrm{pcm}$ for the full core homogeneous cases and the heterogeneous 2D 
fuel assembly slice. The PROTEUS-SN estimate of control rod worth for the homogeneous case is $6072 \mathrm{pcm}$ (1012 pcm per each of the six symmetric primary control rods) which agrees well with the MCNP calculation of $6065 \mathrm{pcm}$ (1010 pcm per primary control rod). These calculations do not include the heterogeneity effect of the control rod. The MCNP calculations for the 3D heterogeneous control rods in and out cases estimate the total heterogeneity effect of the six control rods is $251 \mathrm{pcm}$ (42 pcm per primary control rod).

Table 3.4.1 Eigenvalue verification for VTR cases

\begin{tabular}{|l|l|l|}
\hline \multicolumn{1}{|c|}{ VTR Case } & $\begin{array}{l}\text { MCNP } \\
\text { (pcm uncertainty) }\end{array}$ & $\begin{array}{l}\text { PROTEUS-SN 33g } \\
\text { (pcm diff from MCNP) }\end{array}$ \\
\hline 2D het fuel assembly & $1.74035(7)$ & $1.74160(125)$ \\
\hline $\begin{array}{l}\text { 3D homogeneous core - } \\
\text { control in }\end{array}$ & $1.02761(6)$ & $1.02646(-115)$ \\
\hline $\begin{array}{l}\text { 3D homogeneous core - } \\
\text { control out }\end{array}$ & $1.08826(6)$ & $1.08718(-108)$ \\
\hline $\begin{array}{l}\text { Control worth in pcm } \\
\text { (homogeneous core) }\end{array}$ & $6065(9)$ & $6072(7)$ \\
\hline $\begin{array}{l}\text { 3D het control assembly core }- \\
\text { control in }\end{array}$ & $1.03044(5)$ & In progress \\
\hline $\begin{array}{l}\text { 3D het control assembly core - } \\
\text { control out }\end{array}$ & $1.08858(6)$ & In progress \\
\hline $\begin{array}{l}\text { Control worth in pcm } \\
\text { (het control assembly core) }\end{array}$ & $5814(8)$ & In progress \\
\hline
\end{tabular}

The axial power distributions in the focal fuel and control assemblies from the homogeneous full core calculations with gamma transport (MCNP and PROTEUS-SN) were compared in Figure 3.4.1 and Figure 3.4.2. The MCNP active core profile was normalized to the same power level as PROTEUS-SN since MCNP does not use a set power level. The power shapes in the fuel and control assemblies show excellent agreement for the homogenous (control in) cases. 


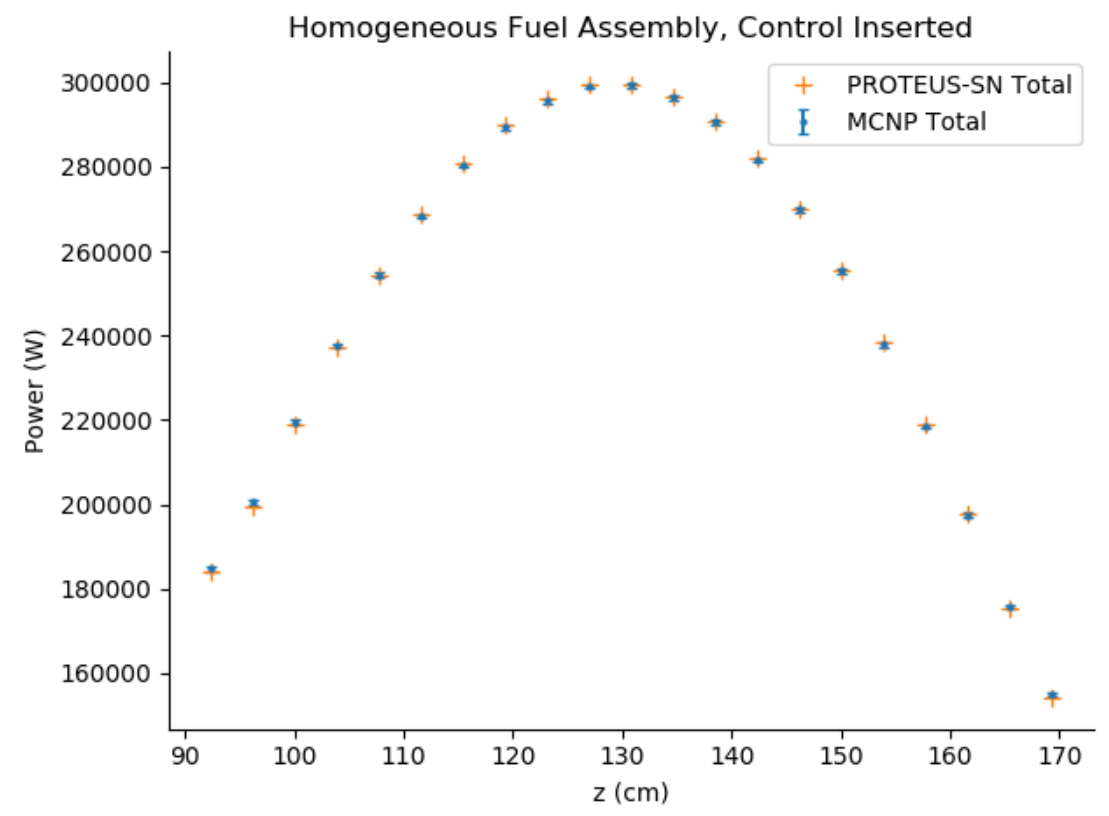

Figure 3.4.1 Verification of axial power profile in fuel assembly (homogeneous full core, control inserted)

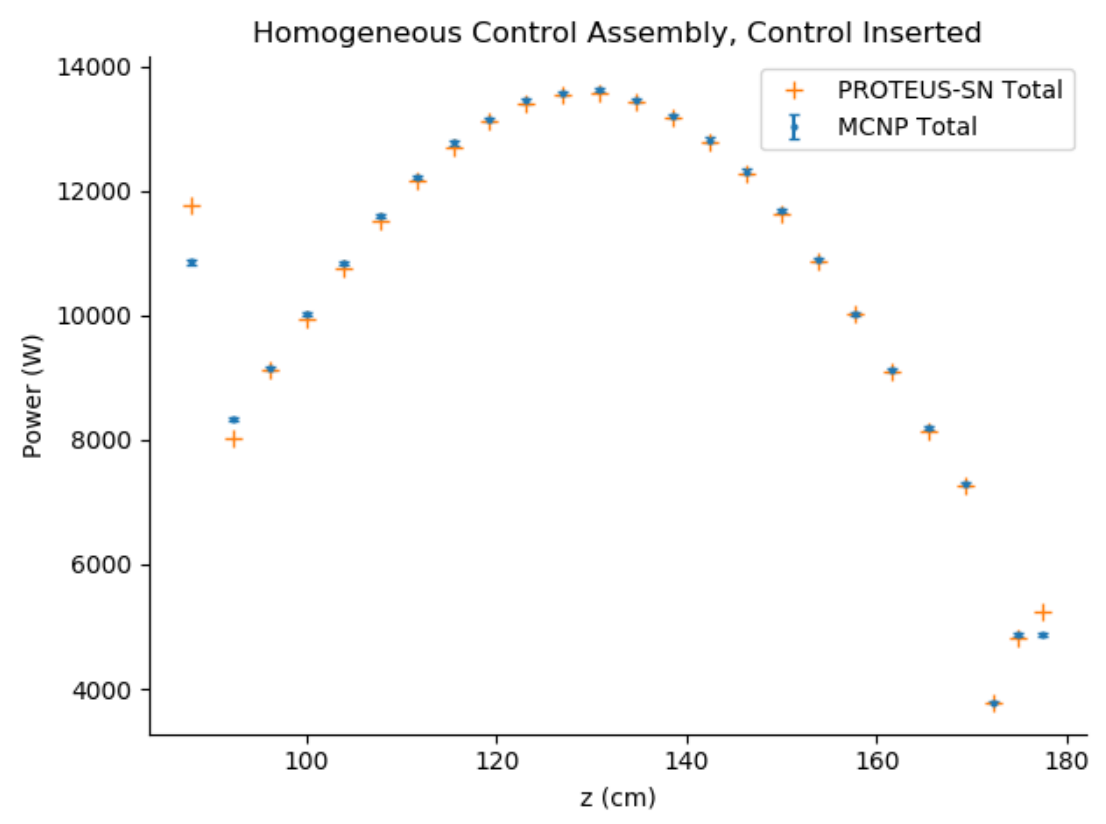

Figure 3.4.2 Verification of axial power profile in control assembly (homogeneous full core, control inserted)

Additionally, the PROTEUS-SN and MCNP radial power profiles near the core-midplane $\mathrm{Z}=(128.9,132.8) \mathrm{cm}$ were plotted in Figure 3.4 .3 (control inserted) and Figure 3.4 .4 (control 
withdrawn). The relative errors for the control inserted case and control withdrawn case for this axial zone are plotted in Figures 4.4.5 and 4.4.6, respectively. The control inserted case shows around a $-5 \%$ error in control assembly power compared to MCNP. The control withdrawn case shows less than $0.5 \%$ error in all assemblies. Larger errors are expected in the control inserted case due to spatially-dependent spectral effects in the vicinity of the strong absorber material when inserted in the active core region.
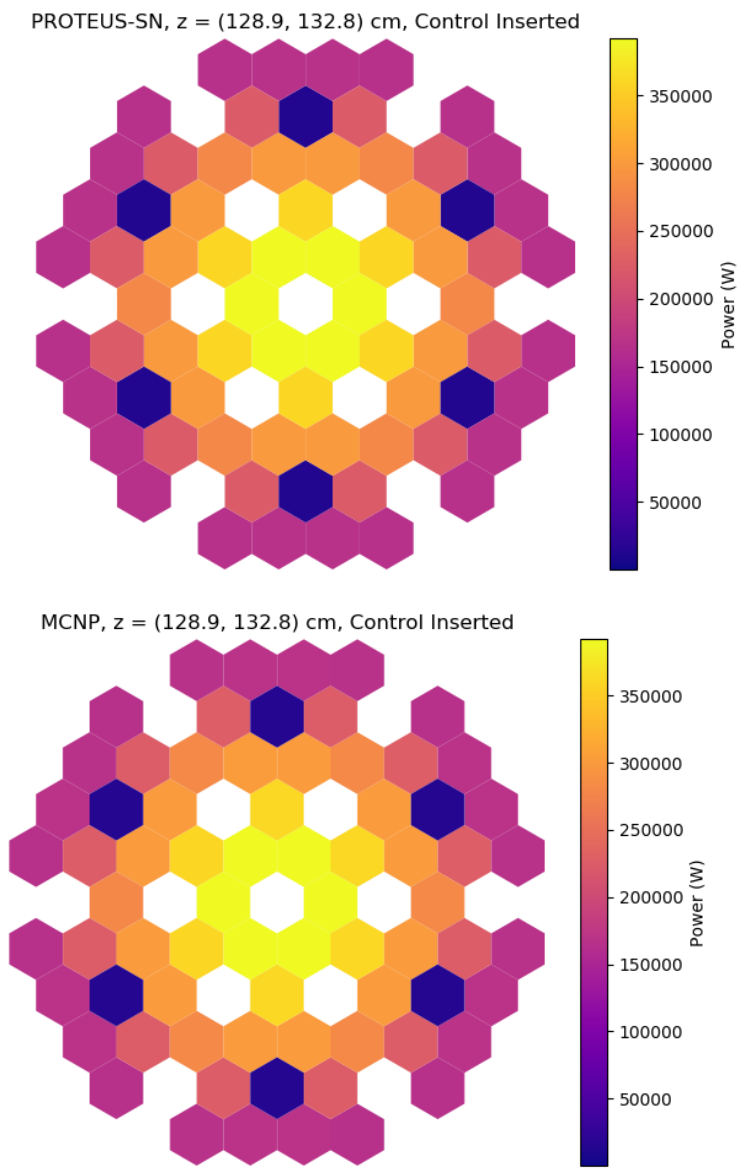

Figure 3.4.3 PROTEUS-SN and MCNP radial power profiles (homogeneous full core, control inserted) 

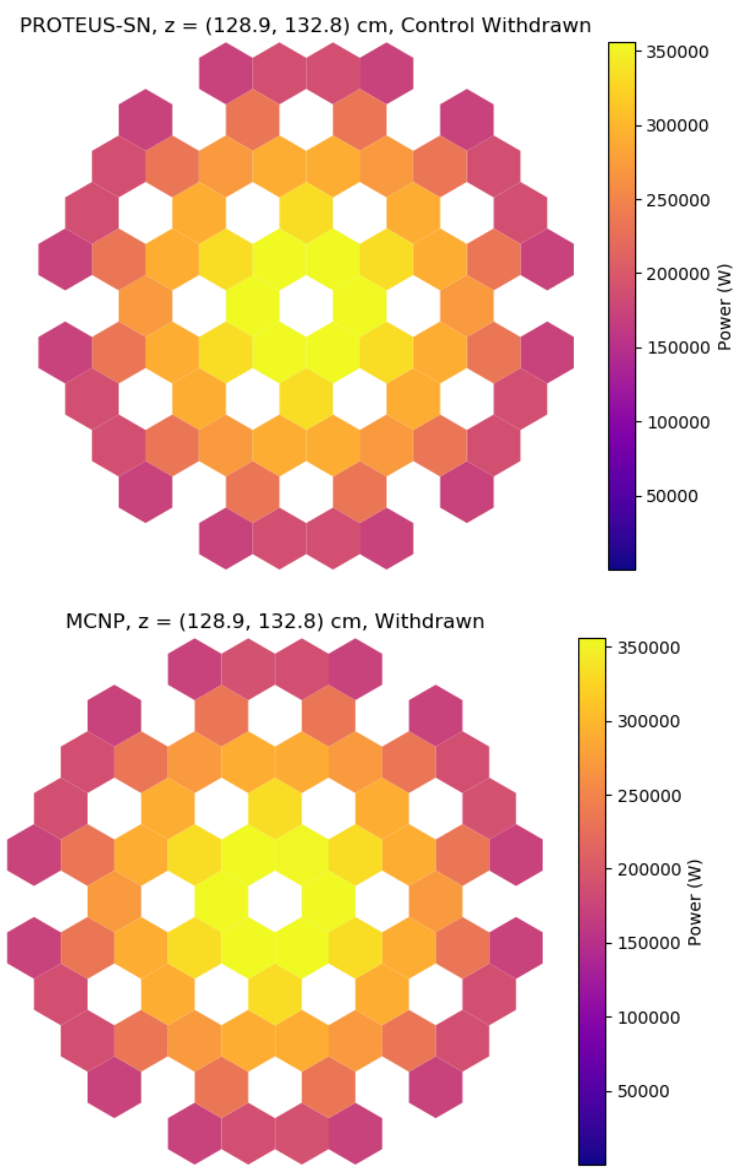

Figure 3.4.4 PROTEUS-SN and MCNP radial power profiles (homogeneous full core, control withdrawn) 


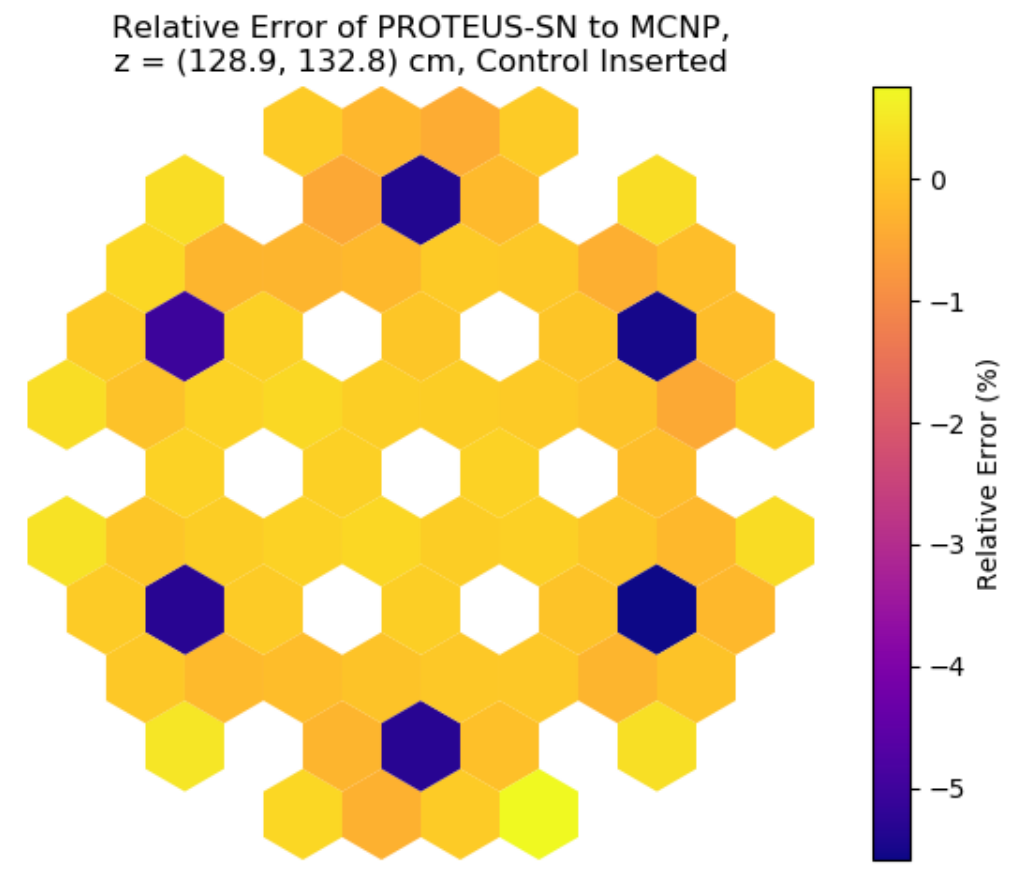

Figure 3.4.5 Relative error in radial power profile in core (homogeneous full core, control inserted)

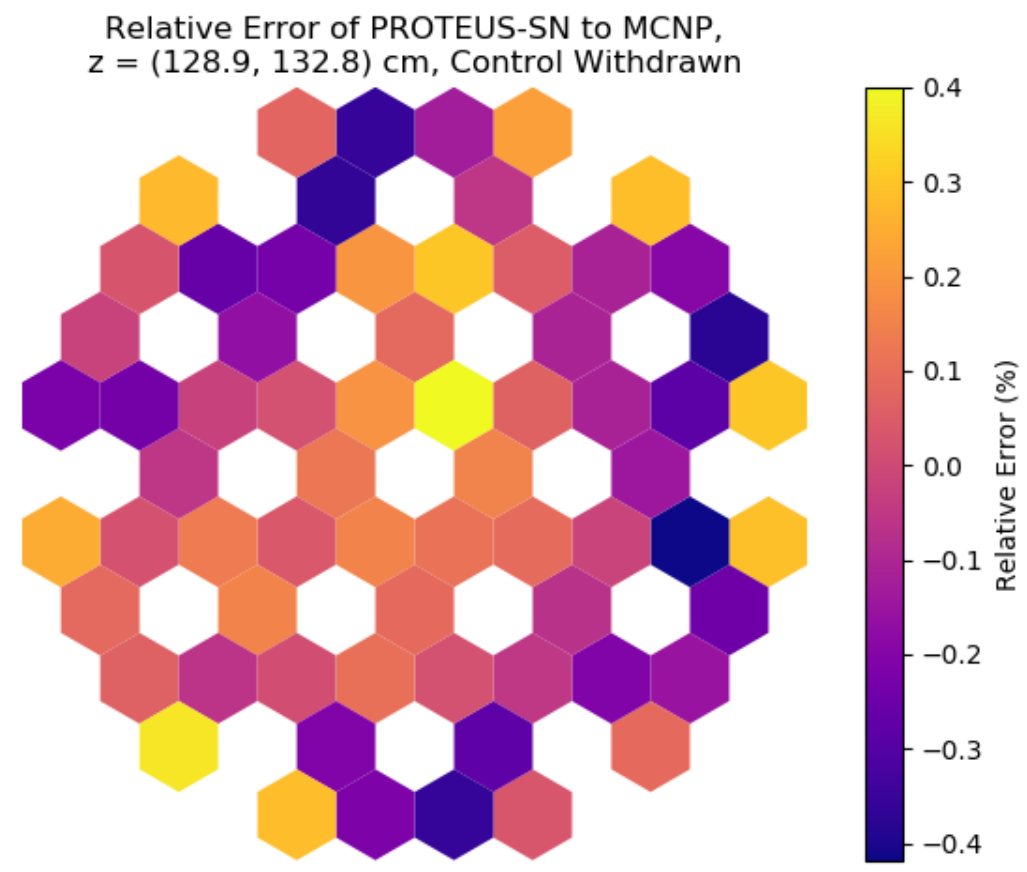

Figure 3.4.6 Relative error in radial power profile in core (homogeneous full core, control withdrawn) 


\subsubsection{Progress on Heterogeneous Assembly Calculations}

The full core VTR calculations with heterogeneous assemblies are in the process of being set up, debugged, and executed on high performance machines. So far, the full core cross sections were generated for both control in and control out configurations, using a two-step procedure in MC2-3/TWODANT as described in Chapter 2, Figure 2.4.1.

For debugging reasons, the simulations for heterogeneous fuel assembly and heterogeneous control assembly have been separated into two cases. The full core with heterogeneous fuel assembly mesh (top-view) is depicted in Figure 3.4.7 and Figure 3.4.8 (zoomed in to see details of fuel assembly). The full core with heterogeneous control assemblies mesh (top-view) is depicted in Figure 3.4.9 and Figure 3.4.10 (zoomed in to see details of fuel assembly). Note the propagation of mesh refinement into neighboring assemblies due to the conformal mesh requirements of PROTEUS.

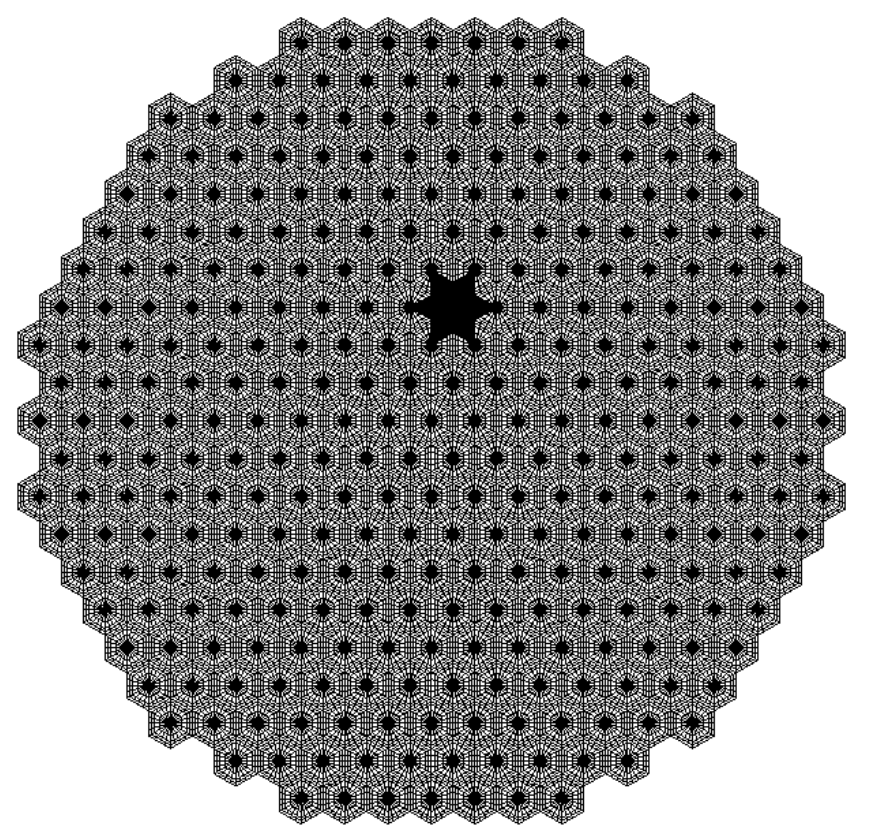

Figure 3.4.7 Full core mesh with detailed fuel assembly 


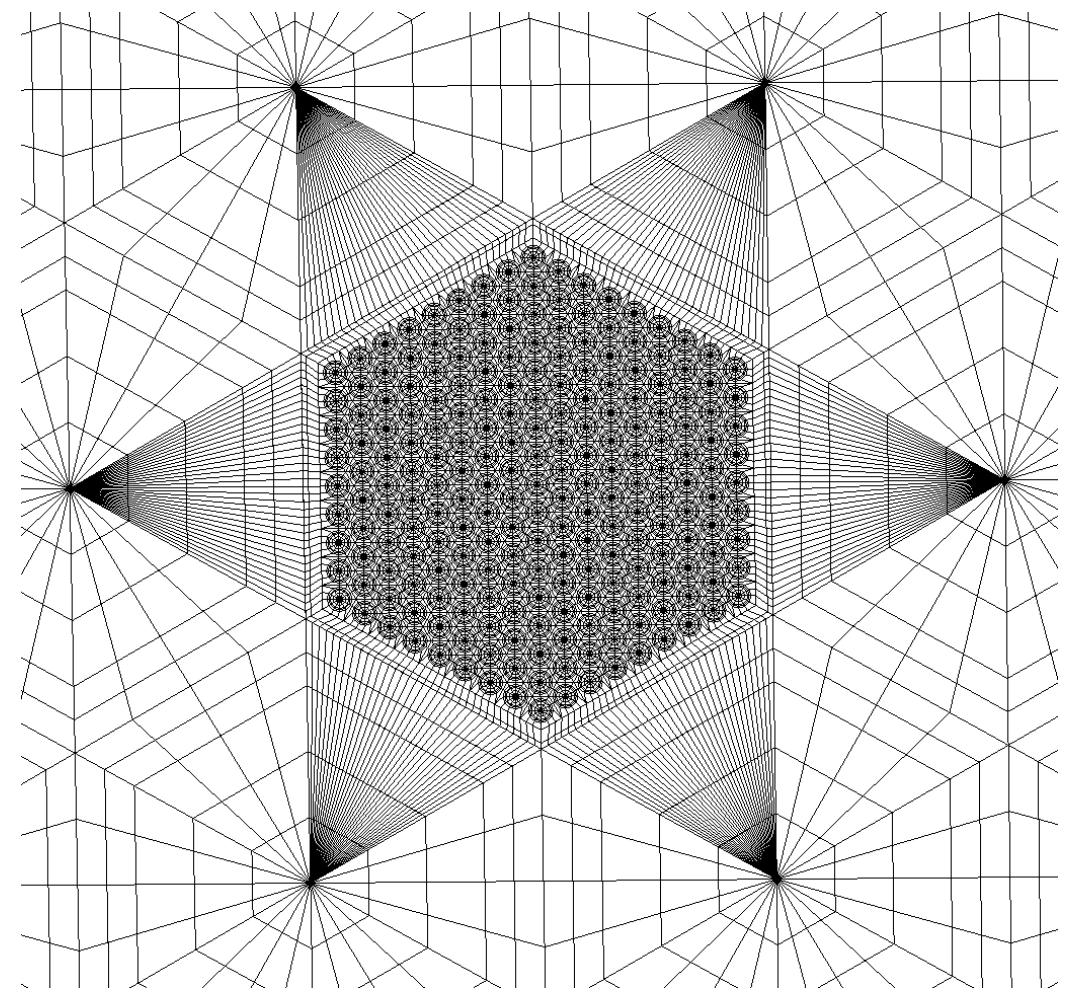

Figure 3.4.8 Full core mesh with detailed fuel assembly (zoomed on fuel assembly)

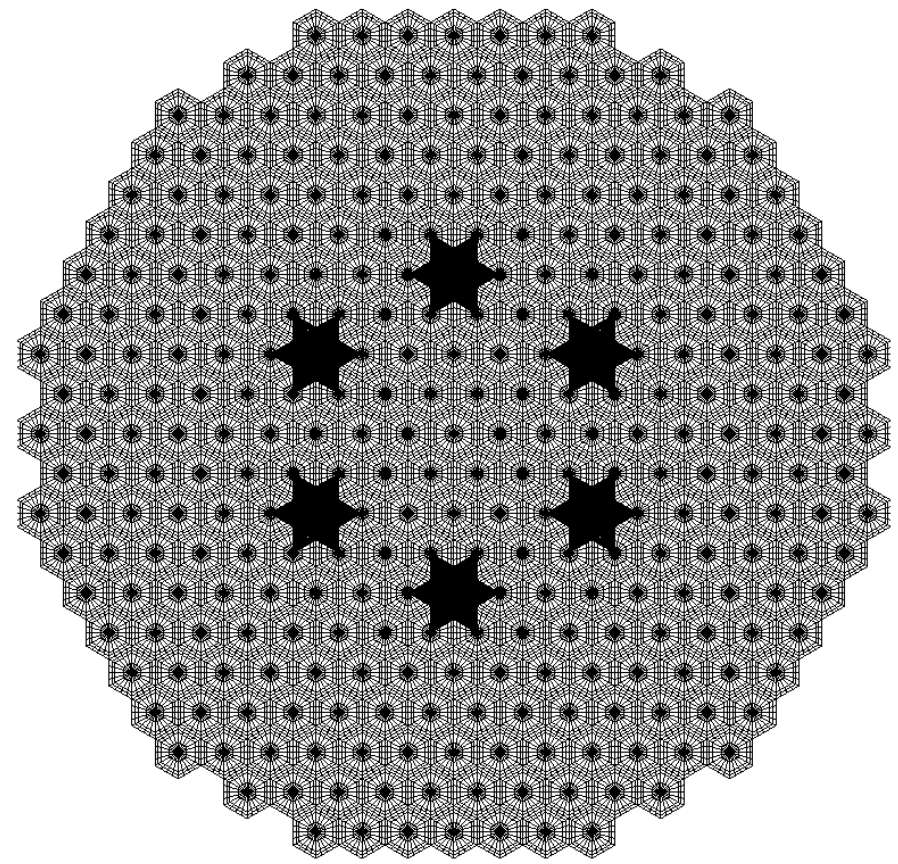

Figure 3.4.9 Full core mesh with detailed control assemblies 


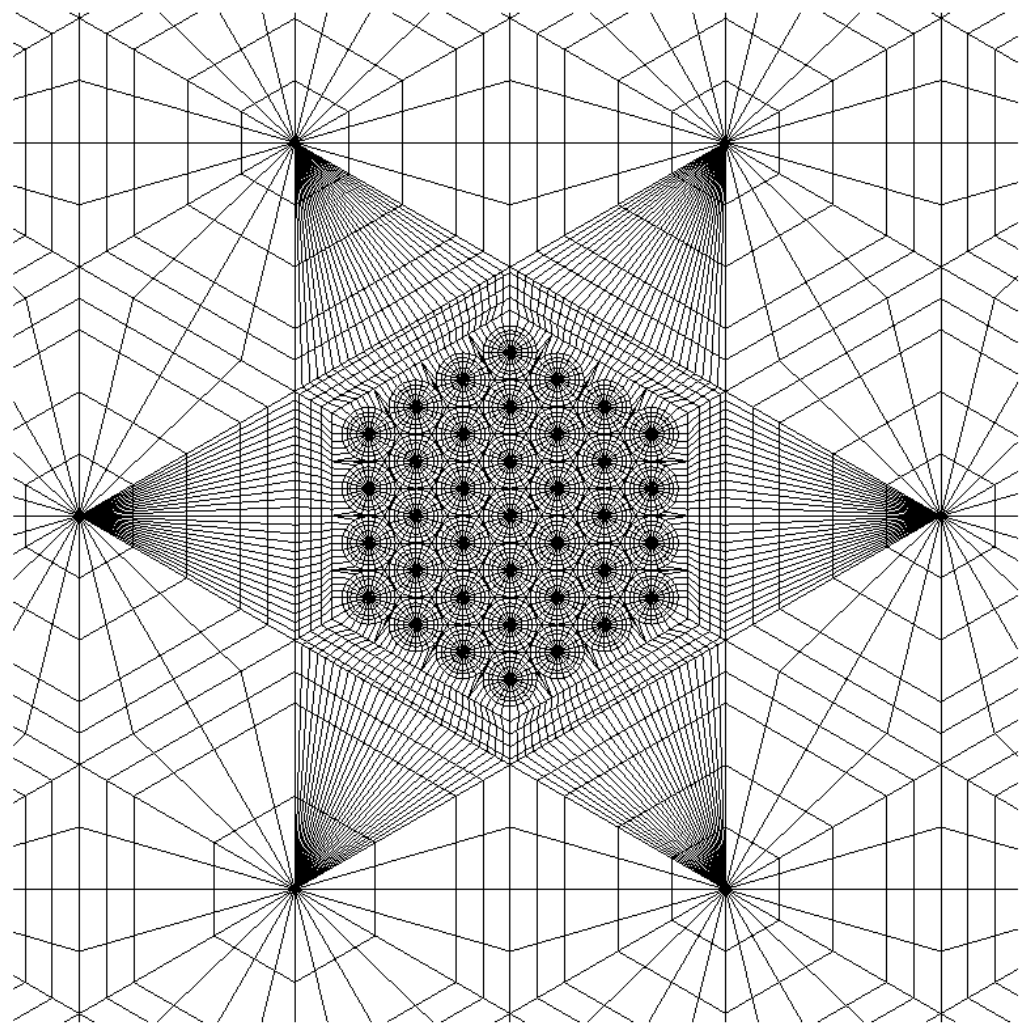

\section{Figure 3.4.10 Full core mesh with detailed control assembly (zoomed on control assembly)}

The full core jobs with heterogeneous assemblies are being debugged. An issue appeared in the 3D heterogeneous simulations at scale, which may be related to the mesh or the cross sections. Due to extraordinarily long queueing times (one month queue times currently, on our allocated resource), it is quite difficult to submit jobs for debugging and then make changes. Once available, the power distributions in a fuel and control assembly will be processed and sent to Nek5000 for simulation of the temperature fields. In the meantime, the MCNP power distributions are used in Nek5000 to complete the analysis.

\subsection{Thermal Hydraulic Calculations}

The Nek5000 calculations for the temperature field are dependent on the power solution as input and require significant computational time. In the Nek5000 CFD model, the $\mathrm{B}_{4} \mathrm{C}$ absorber, cladding, gap and two ducts are modelled explicitly as shown in the top view of the CFD mesh in Figure 3.5.1. As opposed to the neutronics calculations, only the control assembly itself needs to be modeled. The assembly ducts sufficiently insulate the interior of the assembly from the temperature and flow of neighboring assemblies. Adiabatic boundary conditions are places on 
the exterior boundaries. The flow rate of the control rod are determined by the power rate to make sure the outlet flow temperature of the control assembly is consistent with that of fuel assembly. The URANS solver is used for this configuration.

The predicted temperature distribution of the control assembly is shown in Figure 3.5.2 using the power distribution obtained from MCNP (since PROTEUS results were not yet available). The temperature bias due to the power tilt is observed. The control rods closer closest to the center of the core are hotter than the rods closer to the exterior of the core.

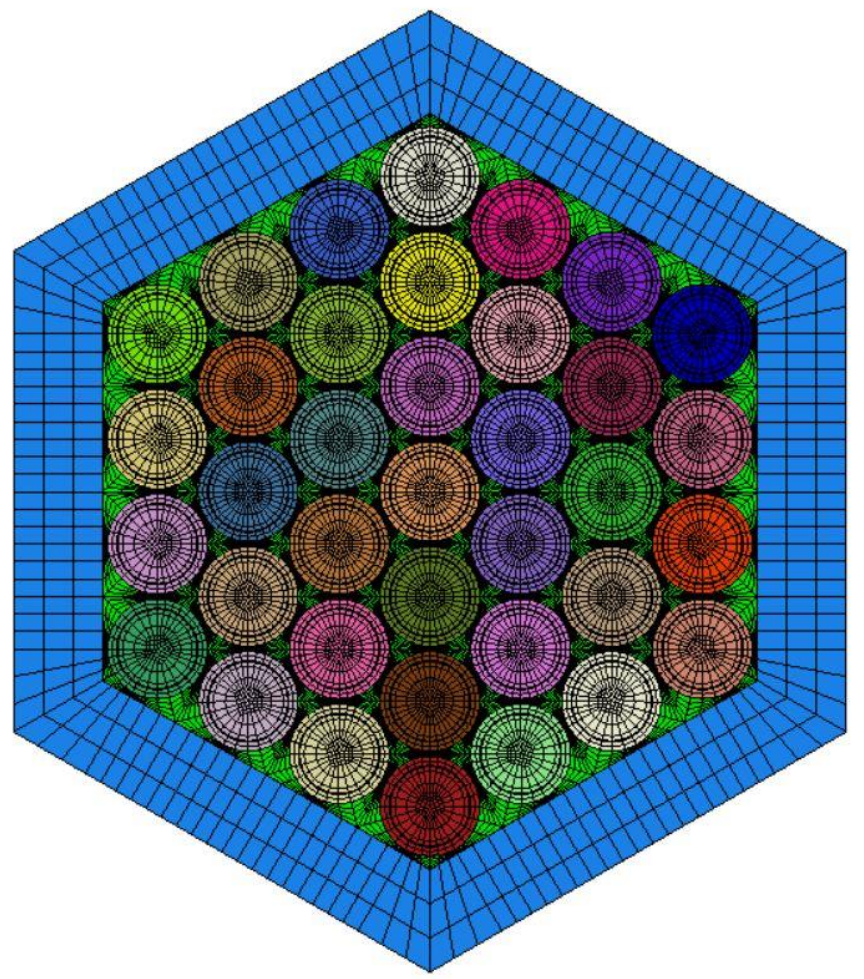

Figure 3.5.1 Thermal hydraulics mesh for control assembly (top view) 


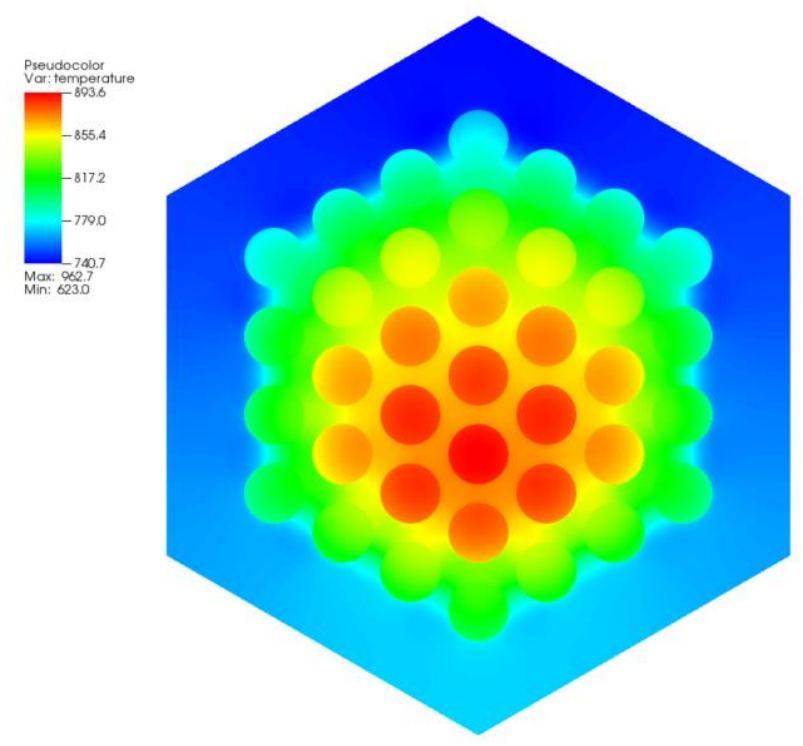

Figure 3.5.2 Temperature distribution of top-most control assembly based on MCNP power distribution

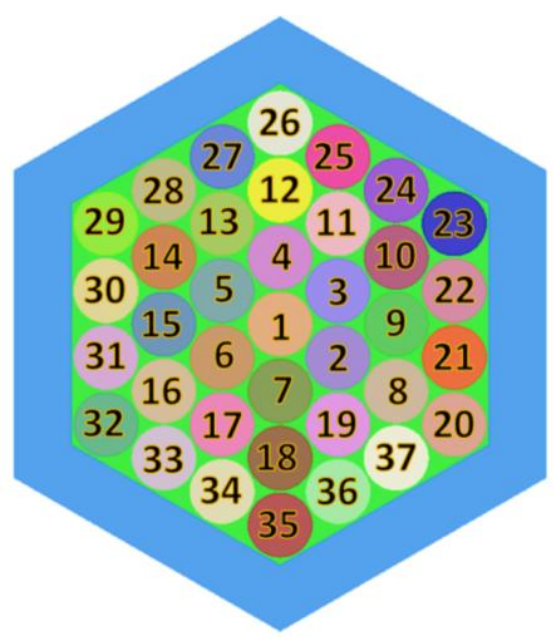

Figure 3.5.3 Pin numbering in control assembly 


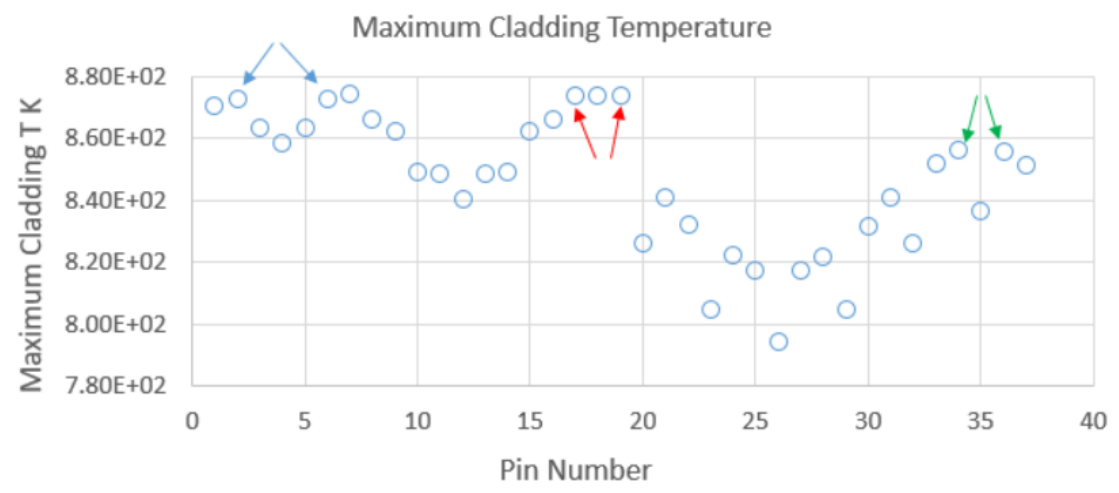

Figure 3.5.4 Maximum cladding temperatures in control assembly (MCNP power)

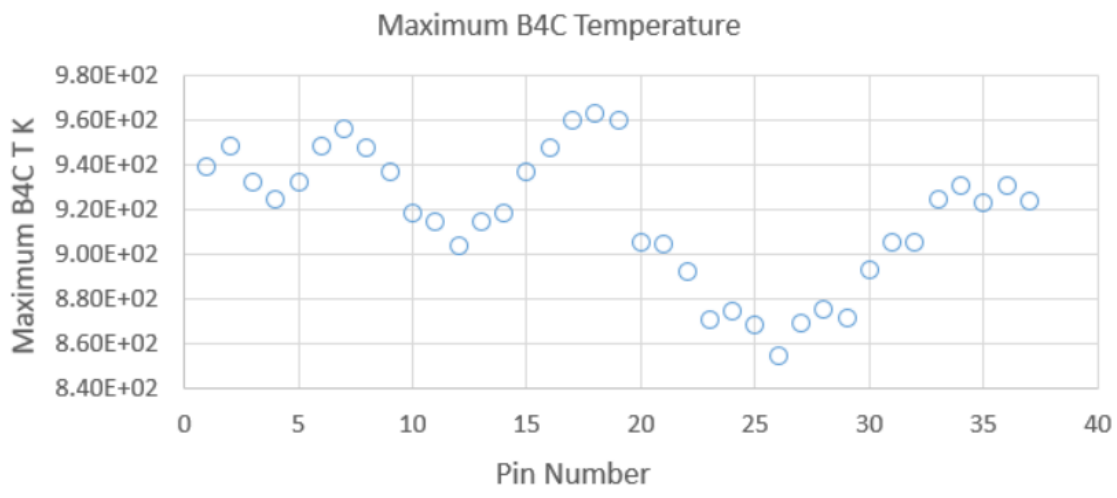

Figure 3.5.5 Maximum absorber temperatures in control assembly (MCNP power)

The control pin numbering is shown in Figure 3.5.3. Detailed information on the peak cladding and B4C absorber temperatures of each pin are shown in Figure 3.5.4 and 3.5.5. Nek5000 predicts both the magnitude and location of the peak cladding and B4C temperatures (874.43K and $962.67 \mathrm{~K})$. The temperature shows bilateral symmetry due to the geometric symmetry of the core. The peak temperature prediction with the high fidelity Nek5000 tool should be more accurate than results from sub-channel or other legacy codes previously used for peak temperature estimation. Based on current results, the peak B4C temperature is much lower than its melting temperature. The information obtained in this study can be used to inform the control rod design in the VTR program. 


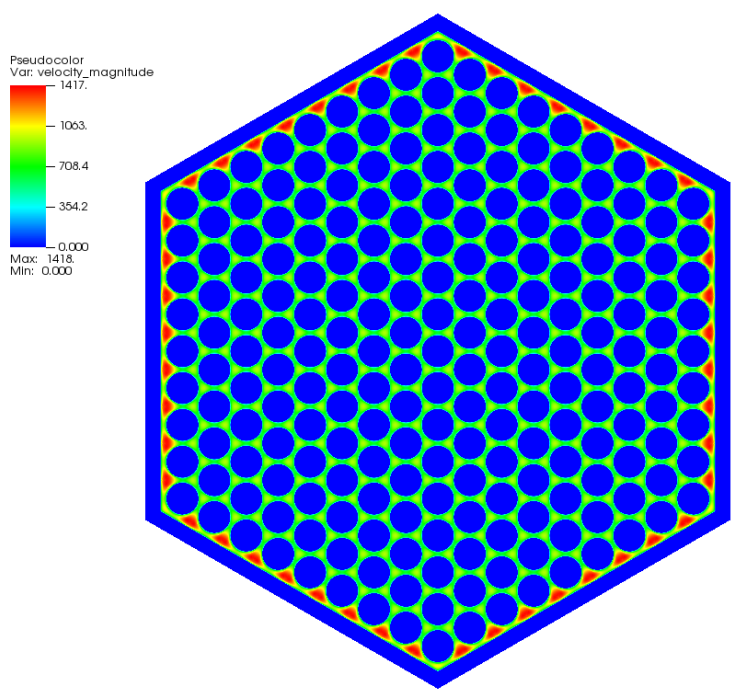

a) velocity

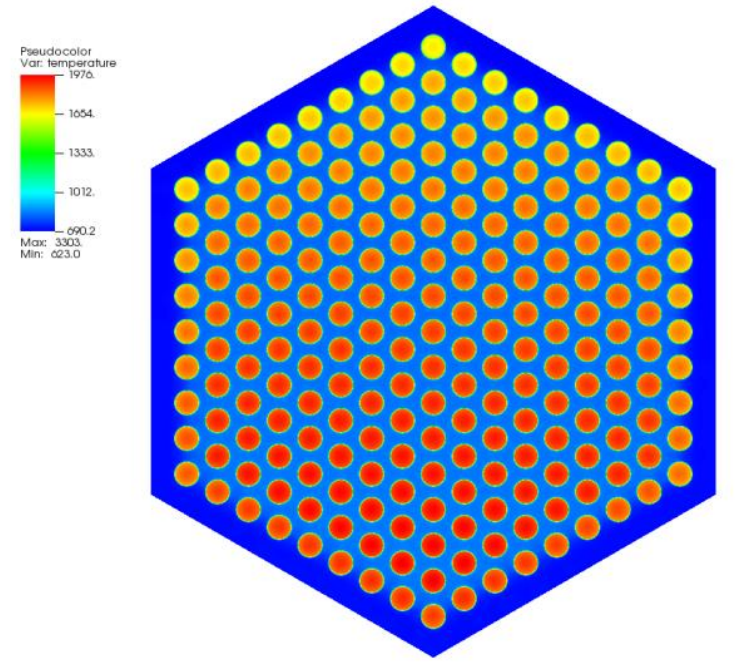

a) temperature

Figure 3.5.6 Velocity and temperature distribution in fuel assembly (MCNP power)

The predicted temperature distribution of the control assembly is shown in Figure 3.5.6 using the power distribution obtained from MCNP. Similar to the simulation of control rod, the temperature bias due to the power tilt is observed. The fuel rods closer closest to the center of the core are hotter. The maximum cladding temperature and maximum fuel temperature rise in the fuel assembly are shown in Figure 3.5.7 and Figure 3.5.8 respectively. The influence of the corner can still be observed on the maximum cladding temperature where a temperature drop is observed in corner pin. The influence of the corner on the maximum fuel temperature rise is weak. 


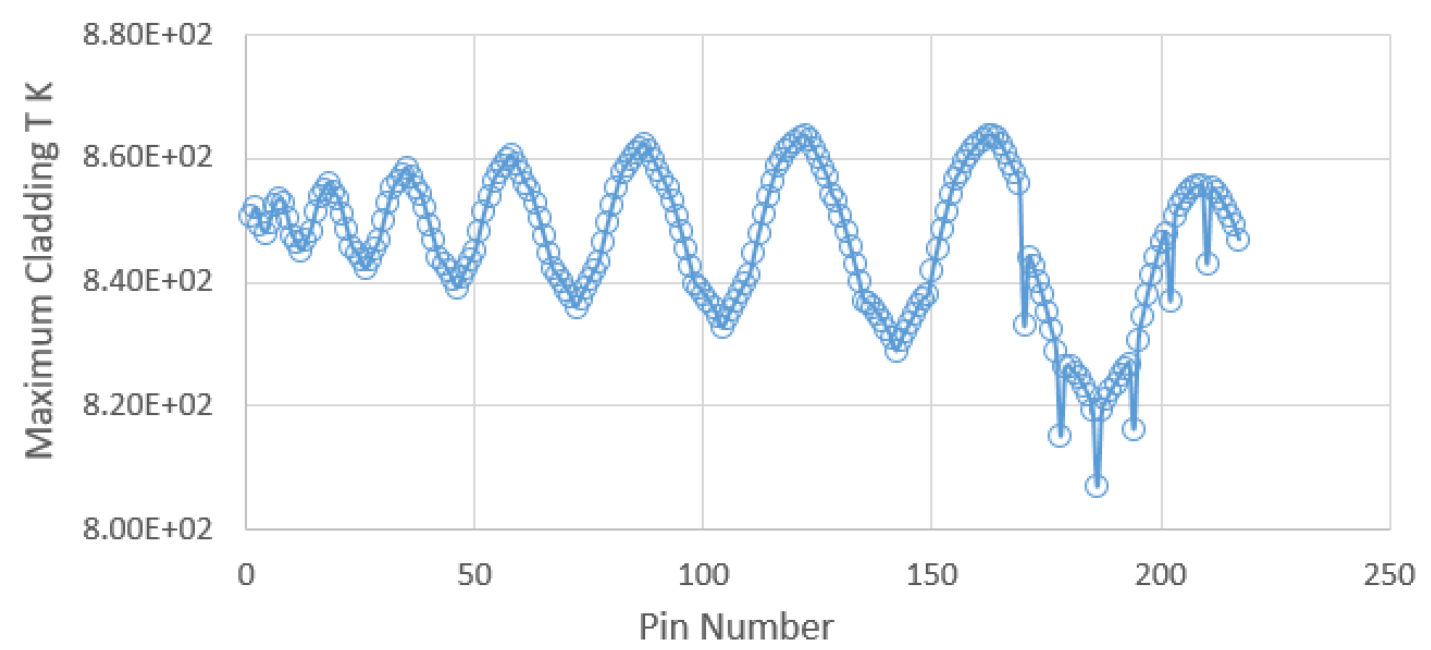

Figure 3.5.7 Maximum cladding temperatures in fuel assembly (MCNP power)

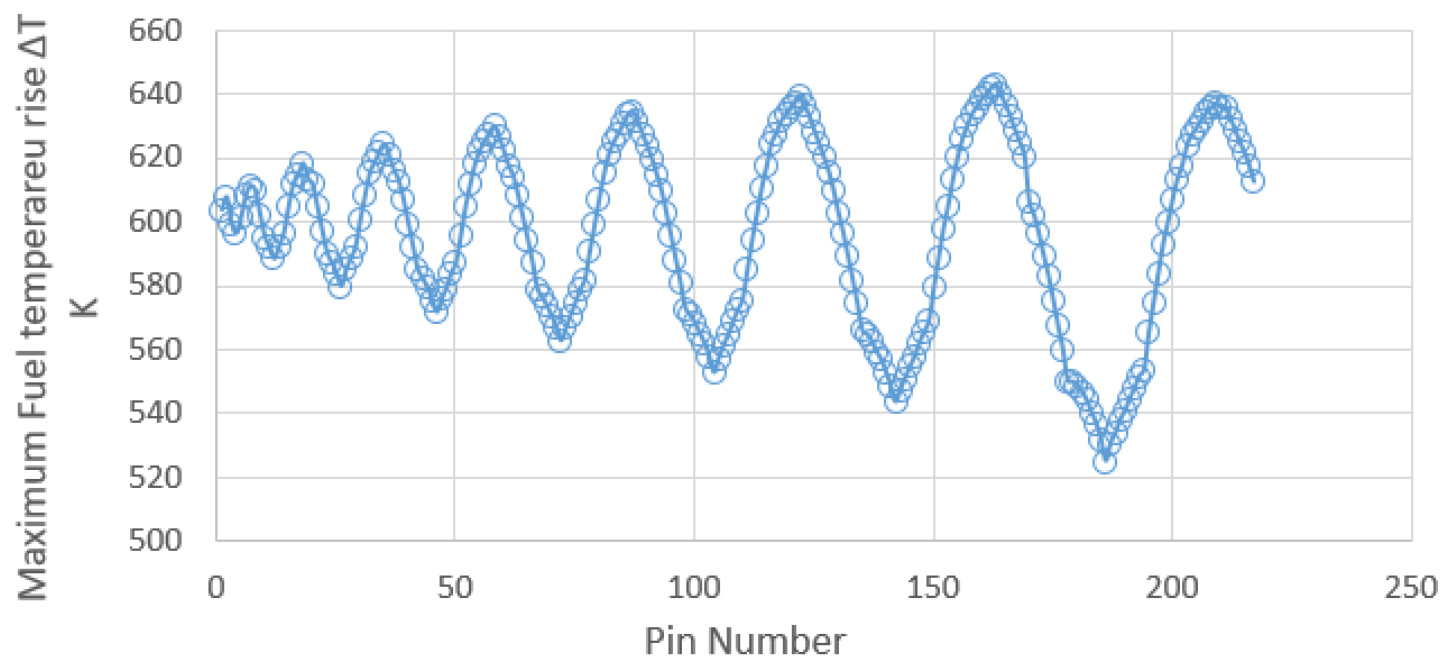

Figure 3.5.8 Maximum fuel temperatures rise in fuel assembly (MCNP power)

\subsection{Summary and Future Work}

The VTR zooming demonstration is partially complete. The hot spot and peak temperatures in the control and fuel assemblies were computed using MCNP for neutronics and Nek5000 for temperature calculation due to issues debugging the full core PROTEUS 3D heterogeneous core model at scale. Homogeneous full core and smaller heterogeneous neutronics verification cases were successfully performed to test the cross sections and meshes between MCNP and PROTEUS-SN. Additional verification cases are necessary for the heterogeneous control 
configurations. Excellent agreement of the eigenvalue calculation was observed between MCNP and PROTEUS for the cases performed. The axial and radial power profiles for the control assembly also compared favorably for the homogeneous core case, showing that the gamma transport capability is implemented correctly. Debugging is still needed for the full core neutronics cases with heterogeneous assemblies, which requires significant wait time due to long queuing time on the high performance machines. The control rod worth was computed with MCNP from the cases with control rods in and control rods out. The power distribution from the "control rods in" case was transferred to Nek5000 for the control assembly for temperature calculation. The power distribution from the control rods out case was transferred to Nek5000 for the fuel assembly. The PROTEUS-SN solutions are not expected to differ significantly from MCNP solutions and will be compared when available. A DOE-Office of Science ALCC allocation was awarded on ALCF's Theta machine (July 2019 - June 2020) to perform the calculations in this work. 


\section{Conclusions}

This document summarizes the extension and application of SHARP tools for various fast reactor types (LFR, SFR). The multiphysics coupling methods developed for SFRs in FY17FY18 were employed in this work.

In the lead-cooled fast reactor (LFR) domain, first-of-a-kind hot channel factor (HCF) estimation with high fidelity codes (PROTEUS/Nek5000) was successfully demonstrated in this study. Selected HCF were computed and compared with SFR data (AFR-100, EBR-II). The findings confirm that different reactor types, design parameters and uncertainties lead to different HCFs. Careful estimation of HCF for a specific design is necessary. In addition to improvement in $\mathrm{HCF}$ accuracy, high fidelity tools generate substantial data to both support the HCF calculation and help the designer better understand the mechanism of the impact from these uncertainties. For example, the impact of cladding thickness manufacturing tolerance resulted in non-intuitive effects in the corner pins of the LFR assembly. To restate, during the process of the HCF calculation, the reactor design is analyzed carefully with a high fidelity model. Some improvement or optimization may be found to be necessary. This procedure of computing HCF using high fidelity models shows promise and flexibility for being repeated for any arbitrary reactor of choice, since these tools are targeted to solve many reactor types and geometries.

In the sodium-cooled fast reactor (SFR) domain, progress was made towards extending the previously demonstrated SHARP zooming capability to non-fueled assemblies. In particular, a gamma transport capability was implemented in high fidelity PROTEUS solvers in order to accurately account for heat deposition caused by gamma particles, which accounts for $\sim 10 \%$ of total core power. The SHARP zooming capability demonstration in FY18 showed that ducted assemblies such as those in a conventional SFR design can be modeled as isolated assembles in thermal hydraulics to the insulation provided by the ducts. Neutronics verification cases were carried out for a candidate Versatile Test Reactor (VTR) design using the new gamma transport capability in PROTEUS for homogenized geometry. Comparisons were made with continuous energy MCNP calculations and shown to agree well. The models for the full core design with heterogeneous control and fuel assemblies are in progress, and MCNP calculations were successfully completed. Thermal hydraulic calculations to compute the maximum temperature in the control assemblies were performed using the power distributions from MCNP. Once available, the deterministic power solutions will be compared with MCNP and if different, transferred to Nek5000 for analysis. 


\section{References}

[Alcouffe 1984] R. E. Alcouffe, F. W. Brinkley, D. R. Marr, and R. D. O'dell, “User's Guide for TWODANT: A Code Package for Two-Dimensional, Diffusion-Accelerated Neutral Particle Transport,” LA-10049-M, Los Alamos National Laboratory (1984).

[Aoki 1963] Aoki, S., A consideration on the heat transfer in liquid metal. Bulletin of the Tokyo Institute of Technology, 63-73, 1963

[Cappia 2020] F. Cappia, B.D. Miller, J.A. Aguiar, L. He, D.J. Murray, B.J. Frickey, J.D. Stanek, J.M. Harp, Electron microscopy characterization of fast reactor MOX Joint Oxyde-Gaine (JOG),Journal of Nuclear Materials, Volume 531,2020,151964

[Cheng 2004] X. Cheng, A. Batta, H.Y. Chen, N.I. Tak, "Turbulent heat transfer to heavy liquid metals in circular tubes," 2004 ASME Heat Transfer and Fluids Engineering Summer Conference, Charlotte, NC, USA, July 11-15 (2004)

[Cheng 2006][1] X. Cheng, N. Tak, CFD analysis of thermal-hydraulic behavior of heavy liquid metals in sub-channels, Nucl. Eng. Des. 236. p. 1874-1885. 2006

[Cheng 2006][2] X. Cheng, N. Tak, Investigation on turbulent heat transfer to lead-bismuth eutectic flows in circular tubes for nuclear applications, Nuclear Engineering and Design. 236. p.385-393.2006

[Fischer 2008] P. F. Fischer, J. W. Lottes, and Stefan G. Kerkemier, Nek5000 Web Page, http://nek5000.mcs.anl.gov, 2008.

[Garnier 1979] Garnier, J.E., \& Begej, S. (1979). "Ex-reactor determination of thermal gap and contact conductance between uranium dioxide: zircaloy-4 interfaces Stage I: low gas pressure", (NUREG/CR--0330), United States

[Grandy 2013] C. Grandy, T.K. Kim, et al, "Advanced Fast Reactor - 100 - Design Overview”, Proceedings of FR-13: Fast Reactor and Related Fuel Cycles, Paris, France, March 4-7, 2013.

[Grasso 2019] G. Grasso, A. Levinsky, F. Franceschini, P. Ferroni, “A MOX-fuel core configuration for the Westinghouse Lead Fast Reactor," ICAPP 2019 - International Congress on Advances in Nuclear Power Plants, France, 2019, May 12-15

[Grudzinski 2019] J Grudzinski, N Wozniak, TS Lee, R Vilim, A Tentner, A Karahan, TK Kim, FY19 Summary Report for US and Japan Bilateral Collaborations, ANL/NSE$19 / 36,2019$

[Hader 2014] J. S. Hader, E. R. Shemon and C. H. Lee, "Initial Verification of Heterogeneous Multigroup Cross Sections in MC2-3/PROTEUS", Transactions of ANS Winter Meeting 2014, Anaheim, CA, November 9-13, 2014.

[Ho 1977] C.Y. Ho, T. Chu, Electrical Resistivity and Thermal Conductivity of Nine Selected AISI Stainless Steels, Tech. Rep. DTIC Document, 1977.

[Ikusawa 2017] Yoshihisa Ikusawa, Koji Maeda, Masato Kato, Masayoshi Uno. (2017) "Oxide-Metal Ratio Dependence of Central Void Formation of Mixed Oxide Fuel Irradiated in Fast Reactors," Nuclear Technology 199:1, pages 83-95 
[Ishii 1977] Tetsuya Ishii, Tomoyasu Mizuno,An investigation of the thermal conductivity of Cs2 Mo04,Journal of Nuclear Materials,Volume 247,1997,Pages 82-85.

[Jarrett 2016] M. G. Jarrett, E. R. Shemon and C. H. Lee, "Heterogeneous Multigroup Cross Sections for Fast Reactor Calculations with MC2-3/PROTEUS", Proceedings of PHYSOR 2016, Sun Valley, ID, May 1-5, 2016.

[Jischa 1979] Jischa, M., Rieke, H. B., About the prediction of turbulent Prandtl and Schmidt numbers from modeled transport equations, International Journal of Heat and Mass Transfer, 22, 1547-1555,1979

[Jung 2019] Y. S. Jung and C. H. Lee, "Updates and Verifications of the PROTEUS System in FY19,” ANL/NSE-19/34, Argonne National Laboratory, September 30, 2019.

[Kays 1994] W. Kays, Turbulent Prandtl number - where are we, J. Heat Transfer - Trans. ASME 116 (1994) 284-295.

[Khorasanov 2013] Georgy L. Khorasanov and Anatoly I. Blokhin, "Benefits in Using Lead208 Coolant for Fast Reactors and Accelerator Driven Systems", Current Research in Nuclear Reactor Technology in Brazil and Worldwide, Amir Zacarias Mesquita, IntechOpen, DOI: 10.5772/52321. (February 6th 2013). Available from: https://www.intechopen.com/books/current-research-in-nuclear-reactor-technology-inbrazil-and-worldwide/benefits-in-using-lead-208-coolant-for-fast-reactors-andaccelerator-driven-systems

[Kim 2012] T. K. Kim, C. Grandy, and B. Hill, "A 100 MWe Advanced Sodium-Cooled Fast Reactor Core Concept," PHYSOR 2012 - Advances in Reactor Physics - Linking Research, Industry, and Education, Knoxville, Tennessee, USA, April 15-20, 2012.

[Kirshenbaum 1961] Kirshenbaum, A. \& Cahill, J. \& Grosse, A. "The density of liquid lead from the melting", Journal of Inorganic and Nuclear Chemistry, 22. pp33-38 (1961). Available from: https://www.researchgate.net/publication/243637448_The_density_of_liquid_lead_fro m_the_melting

[Ku 1994] J. Y. Ku, L. K. Chang and D. Mohr, "Analysis of IFR Driver Fuel Hot Channel Factors," Fourth International Topical Meeting on Nuclear Thermal Hydraulics, Operation and Safety, Taipei, Taiwan, April 5-8, 1994.

[Lai 2019] Lai, J. K., Busco, G., Merzari, E., and Hassan, Y. A. (October 8, 2019). "Direct Numerical Simulation of the Flow in a Bare Rod Bundle at Different Prandtl Numbers." ASME. J. Heat Transfer. December 2019; 141(12): 121702.

[Lee 2012] C. H. Lee and W. S. Yang, "MC2-3: Multigroup Cross Section Generation Code for Fast Reactor Anaysis,” ANL/NE-11-41 Rev. 1, Argonne National Laboratory, January, 2012.

[Lubarsky 1955] B. Lubarsky, S.J. Kaufman, Review of experimental investigation of liquid metal heat transfer, in: NACA Technical Note 3366, 1955 
[Mahadevan 2014] V. S. Mahadevan, et al, "SHARP Coupled Multiphysics Toolkit for Reactor Analysis," Proceedings of ICAPP 2014, Charlotte, NC, April 6-9, 2014.

[Melis 1993] J.-C. Melis, J.-P. Piron, L. Roche, "Fuel modeling at high burn-up: recent development of the GERMINAL code," Journal of Nuclear Materials, Volume 204,1993,Pages 188-193

[Merzari 2015] E. Merzari, E. Shemon, Y. Yu, J. Thomas, A. Obabko, R. Jain, V. Mahadevan, J. Solberg, R. Ferencz, R. Whitesides et al, "Multi-Physics Demonstration Problem with the SHARP Reactor Simulation Toolkit", Argonne National Laboratory Technical Report ANL-NE-15/44, December 21, 2015.

[Myong 1989] Hyon Kook MYONG, Nobuhide KASAGI, Masaru HIRATA, "Numerical Prediction of Turbulent Pipe Flow Heat Transfer for Various Prandtl Number Fluids with the Improved k- $\varepsilon$ Turbulence Mode"l, JSME international journal. Ser. 2, Vol.32, No.4, p.613, 1989

[Reynolds 1975] A.J. Reynolds, "The Prediction of Turbulent Prandtl and Schmidt Numbers," Int. J. Heat Mass Trans., 18 (1975), pp. 1055-1069

[Rieneiskii 1999] A. RINEISKII, "Status of Liquid Metal Cooled Fast Reactor Technology," IAEA-TECDOC-1083, International Atomic Energy Agency, 1999

[Shemon 2014] E. R. Shemon, C. H. Lee, M. A. Smith and A. Marin-Lafleche, "NEAMS Neutronics: Development and Validation Status", Proceedings of ICAPP 2014, Charlotte, NC, April 6-9, 2014.

[Shemon 2018] E. R. Shemon, Y. Yu, and T. K. Kim, "Applications of SHARP Toolkit to SFR Challenging Problems: Evaluation of Hot Channel Factors and Demonstration of Zooming Capability," ANL/NSE-18/4, Argonne National Laboratory, September 30, 2018.

[Smith 2017] A. L. Smith, et al, "Structural and thermodynamic study of dicesium molybdate $\mathrm{CS}_{2} \mathrm{Mo}_{2} \mathrm{O} 7$ : Implications for fast neutron reactors", J. Solid State Chemistry 253 (2017) 89-102.

[Sobolev 2007] V. Sobolev, Thermophysical properties of lead and lead-bismuth eutectic, Journal of Nuclear Materials, Volume 362, Issues 2-3, 2007,Pages 235-247

[Sprague 2010] M. A. Sprague, "A comparison of Nek5000 and OpenFOAM for the DNS of Turbulent Channel Flow”, Presentation at Nek5000 Users Meeting, Argonne National Laboratory, December 10, 2010.

[Watts 2013] D.J. Watts, Isotope Mixes, “Corresponding Nuclear Properties and Reactor Design Implications of Naturally Occurring Lead Sources", Naval Postgraduate School Thesis (2013). 


\section{Argonne}

\section{Nuclear Science and Engineering Division}

Argonne National Laboratory

9700 South Cass Avenue, Bldg. 208

Argonne, IL 60439

www.anl.gov 